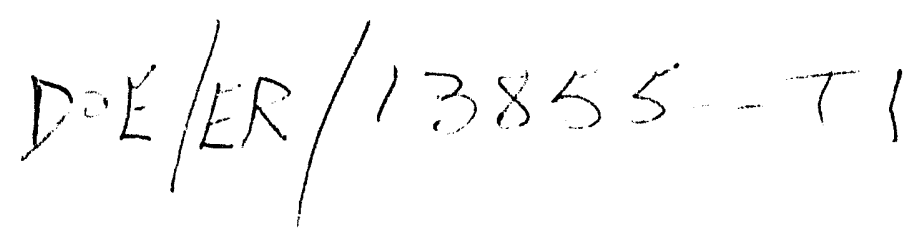

Final Report

DOE/ER/13855--TI

\title{
Stable Isotope Studies
}

DE93 000908

Contracts: AT(11-1)-3127, E(11-1)-3127, EY-76-S-02-3127

DE-AC02-80ER10612, DE-FG02-88ER13855

between

Division of Research and Office of Basic Energy Sciences

of

U. S. Atomic Energy Commission

U. S. Energy Research and Development Administration

U.S. Department of Energy

and

Research Foundation of the City University of New York

for and in conjunction with

Brooklyn College of the City University of New York

and with

Research Foundation of the State University of New York

for and in conjunction with

State University of New York at Stony Brook

Takanobu Ishida

Principal Investigator

March 1, 1972 - February 29, 1992 


\section{Table of Contents}

page

Acknowledgement $\quad$ iii

Summary $\quad$ v

Personnel ix

Curriculum Vitae xiii

Publication $\quad$ Xv

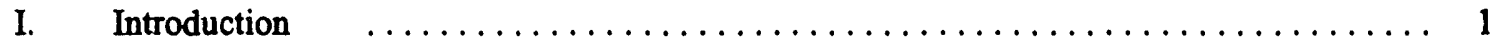

II. Correlation of isotope effects with molecular structures and molecular forces $\ldots, \ldots \ldots 5$

II-A. Development of theoretical tools $\ldots \ldots \ldots \ldots \ldots \ldots \ldots \ldots \ldots$

II-A-1. Direct orthogonal polynomial approximations $\ldots \ldots \ldots \ldots \ldots 6$

II-A-2. Orthogonal expansion coupled with diagonal approximation $\ldots \ldots .8$

II-B. Correlation studies $\quad \ldots \ldots \ldots \ldots \ldots \ldots \ldots \ldots \ldots \ldots \ldots \ldots$

II-B-1. End-atom isotope effects $\ldots \ldots \ldots \ldots \ldots \ldots \ldots \ldots \ldots \ldots \ldots$

Deviations from the first rule of the mean $\quad \ldots \ldots \ldots \ldots \ldots 14$

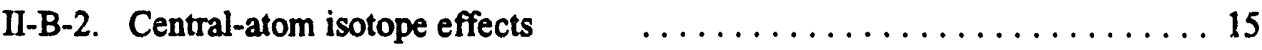

Isotope effects at the center of highly symmetric molecules $\ldots \ldots 16$

III. Correlation of zero-point energy with molecular structures and molecular forces $\ldots \ldots 19$

III-A. Zero-point energy as an explicit function of molecular structures and forces . . 19

III-A-1. Development of mathematical tools $\quad \ldots \ldots \ldots \ldots \ldots \ldots \ldots$

III-A.2. Zero-point energy of n-alkanes $\quad \ldots \ldots \ldots \ldots \ldots \ldots \ldots \ldots 23$

III-A-3. Hydrogen isotope effects in ZPE and linear frequency sum rules $\ldots \ldots 24$

III-A-4. Additivity for zero-point energy $\quad \ldots \ldots \ldots \ldots \ldots \ldots \ldots \ldots 26$

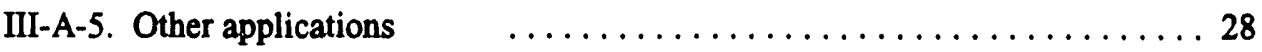

III-B. Isotope effect on the zero-point energy shift upon condensation $\ldots \ldots \ldots 34$

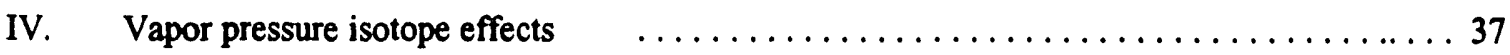

IV-A. Vapor pressure isotope effects in liquid fluoromethanes $\quad \ldots \ldots \ldots \ldots 37$

IV-B. Vapor pressure isotope effects in liquid and solid ammonia $\ldots \ldots \ldots 45$

IV-C. Medium cluster model of liquid for vapor pressure isotope effects $\ldots \ldots 50$

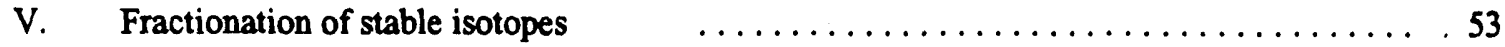

V-A. Fractionation of nitrogen- $15 \quad \ldots \ldots \ldots \ldots \ldots \ldots \ldots \ldots \ldots \ldots$

V-A-1. Equilibrium properties of $\mathrm{NO} / \mathrm{N}_{2} \mathrm{O}_{3}$ system $\quad \ldots \ldots \ldots \ldots \ldots \ldots 5$

V-A-2. Nitrogen-15 fractionation based on the $\mathrm{NO} / \mathrm{N}_{2} \mathrm{O}_{3}$ exchange system $\ldots 55$ 
V-B. Hydrophobic catalysts for hydrogen isotope exchange between dihydrogen

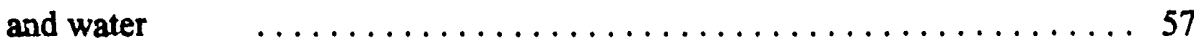

V-B-1. Hydrophobicity of polycrystalline platinum surfaces $\quad \ldots \ldots \ldots \ldots 58$

V-B-2. Chemical poisoning and regeneration of platinum surfaces $\ldots \ldots .59$

V-B-3. Electrochemical modification of platinum surfaces by poly(p-phenylene) .60

V-B-4. Electrochemical modification of platinum surfaces by other organic films . 67

V-B-5. Capacitance measurement for determination of effective surface area of edge plane of pyrolytic graphite $\quad \ldots \ldots \ldots \ldots \ldots \ldots \ldots \ldots 67$

V-C. Transient behavior of square cascade of isotope separation stages $\ldots \ldots 73$

VI. References 


\section{Acknowledgement}

This research has been a collaborative effort with 3 number of senior colleagues, post-doctoral associates, graduate students, and undergraduate students.

Major funding for the research has been provided by the U. S. Atomic Energy Commission, Energy Research and Development Agency and Department of Energy, whose continuous support for this program over the twenty-year period from March, 1972 to February, 1992 has made it possible. The City University of New York supported the program over the period, March, 1972 through August, 1979, with grants for personnel and purchase of instrumeits through its Faculty Research Award Program and for computing time through its Computing Centers. The Max Planck Society of Germany financet Principal Investigator's leave in 1986. The Science and Technology Agency of Japan supported a postdoctoral associate. The Tokyo Club provided a support for a postdoctoral fellow for two years. Brookhaven National Laboratory allowed an access to an isotope ratio mass spectrometer and other major equipment in early seventies.

I express my sincere appreciation to all who have made this research possible.

Takanobu Ishida

Professor of Chemistry

State University of New York

Stony Brook, New York 
[ blank page ] 


\section{Summary}

The research has been conducted by the Principal Investigator, Professor Takanobu Ishida, in collaboration with other colleagues and students in the general area of isotope chemistry and isotope separation during the period, March 1, $1972 \sim$ February 29, 1992. The research has been carried out principally at Brooklyn College of the City University of New York, March, $1972 \sim$ August, 1979, and at the State University of New York at Stony Brook, September, $1979 \sim$ February, 1992. The Principal Investigator spent the period, January, 1986 July, 1986, in Mainz, Germany, as a Max Planck Institute Visiting Scholar.

The research has been in four general areas: (1) correlation of isotope effects with molecular forces and molecular structures, (2) correlation of zero-point energy and its isotope effects with molecular structure and molecular forces, (3) vapor pressure isotope effects, and (4) fractionation of stable isotopes.

\section{(1) Correlation of isotope effects with molecular structure and molecular forces:}

- Mathematical correlation method for the isotopic reduced partition function ratio with molecular structures and molecular forces were developed on the basis of the orthogonal functional theorem, and prototype molecules, $\mathrm{CO}_{2}, \mathrm{HCHO}, \mathrm{CH}_{4}, \mathrm{C}_{2} \mathrm{H}_{4}, \mathrm{C}_{2} \mathrm{H}_{6}$, and $\mathrm{C}_{6} \mathrm{H}_{6}$ were studied.

- For end-atom isotope effects and, in particular, the hydrogen isotope effects:

- The isotopic reduced partition function ratio can be expressed as a sum of contributions of bond-stretching, angle-bending and other intra-molecular motions; the contributions are additive.

- The contributions by stretching and bending motions largely determine the isotope effects.

- The contributions of bond-stretching coordinates are proportional to the stretching force constant, while the contributions of bending motions are not as proportional.

- A simple rule for computing the isotope effects was developed.

- For central-atom isotope effects:

- Bond-stretching forces contribute over seventy percent of total magnitutdes of isotope effects.

- The valence coordinate contributions to the isotopic reduced partition function ratio is not as additive as for the end-atom isotope effects, mainly due to the higher-order kinetic energy interactions.

- For the isotope substitution at the center of highly symmetric molecules, it is possible to obtain an explicit and straightforward correlation of the isotope effects with the molecular parameters. 
- A systematics of D-matrix was developed to predict and identify the sources of deviations from given isotopic rules of the mean.

(2) Correlation of zero-point energy with molecular structures and molecular forces:

- A mathematical tool for the correlation of zero-point energy (ZPE) with the molecular parameters was developed on the basis of the orthogonal functional theorem.

- Linear frequency sum rule was established, which holds approximately but with high precision for the sets of isotopic molecules for which a second-order sum rule holds. The rules for hydrogen isotope substitutions hold with precisions of few wavenumbers.

- Zero-point energy and hydrogen isotope shifts in zero-point energy can be expressed as a sum of contributions of bond-stretching, angle-bending and other molecular motions: The contributions are additive. Tables of transferrable frequency parameters for the additivity were constructed for the homologous series, $n$-alkanes and $n$-alkyl chlorides. Sums of the parameters give the zero-point energy within $0.7 \%$ of exact values.

- Within each main group of periodic table, the contribution of H-X bond-stretching to hydrogen isotope shifts in zero-point energy for the molecule $\mathrm{HX}$ is a linear function of electronegativity of $\mathrm{X}$, where $\mathrm{X}$ is a molecular moiety.

- The effect of London dispersion forces on the isotope effect of zero-point energy shifts due to condensation is expressible as a product of an isotope-independent factor, which only depends on properties of the condensed phase, and an isotope-dependent factor, which only depends on properties of the gas. Rules of thumb for predicting what molecular features would lead to a larger or smaller isotope-shifts in the condensation-shift were developed.

(3) Vapor Pressure Isotope Effects:

- Vapor pressure isotope effects (VPIE) for $\mathrm{D} / \mathrm{H}$ - and ${ }^{13} \mathrm{C} /{ }^{12} \mathrm{C}$-substitutions in liquid methyl fluoride, methylene difluoride, and fluoroform were measured, and the results were compared with the VPIEs in liquid methane. All observed results are explanable in terms of molecular symmetry, relative magnitudes of intermolecular forces in the liquids, and temperature-dependent force constants on the basis of simple cell model.

- Large normal D/H-VPIEs observed in the fluoromethanes are due to the blue-shifts in the C-H stretching frequencies upon condensation.

- Negative curvatures in the plots of $T \ln \left(f_{c} / f_{b}\right)$ vs. $1 / T$ for the $D / H-V P I E$ in the fluoromethanes are due to the blue shifts, the magnitudes of which increase with decreasing temperature. 
- An anomalously large, normal D/H-VPIE in methylene difluoride is also attributable to an extensive interaction between the blue-shifting $\mathrm{C}-\mathrm{H}$ stretching modes and the strong external forces.

- The ${ }^{13} \mathrm{C} /{ }^{12} \mathrm{C}$-VPIEs are inverse, maily due to interactions of the internal modes with hindered rotation.

- Vapor pressure isotope effects for $\mathrm{D} / \mathrm{H}$ - and ${ }^{15} \mathrm{~N} /{ }^{14} \mathrm{~N}$-substitutions in liquid and solid ammonia were measured at temperatures between $163 \mathrm{~K}$ and $243 \mathrm{~K}$.

- The triple points for ${ }^{14} \mathrm{NH}_{3},{ }^{14} \mathrm{ND}_{3}$, and ${ }^{15} \mathrm{NH}_{3}$ were determined.

- The linear dependence of $T \ln \left(f_{c} / f_{2}\right)$ on $1 / T$ is due to the lattice modes, especially, to the hindered rotation.

- The difference in the vapor pressures of $\mathrm{NH}_{3}$ and $\mathrm{ND}_{3}$ is approximately constant between the triple point temperatures of $\mathrm{NH}_{3}$ and $\mathrm{ND}_{3}$.

- The close-packed structure of solid ammonia persists more or less not only through the phase change but also over the liquid temperature range that we studied.

- The external force in liquid ammonia is much more directional on the hydrogen-side of ammonia molecule than on the lone-pair side of the molecule.

- Medium cluster model of liquid was developed. In the model, a cluster consists of a central molecule and a number $(\mathrm{m})$ of indistinguishable molecules that surround the central molecule, and the intermolecular interactions among these molecules are explicitly taken into account.

- The model contains a built-in mechanism by which the observed VPIEs can be represented better than the cell model without significantly increasing the number of force constant parameters.

- As $m$ is increased beyond approximately nine, qualitative features of computational results of the medium cluster model approaches those of the cell model, and the results due to the artifacts of limited cluster sizes become insignificant relative to the experimental precisions attainable by the presently available techniques. A close representation of an observed VPIE data by the cell model should, in principle, be possible through careful attention to details of interaction force constant parameters within the framework of the cell model.

\section{(4) Fractionation of stable isotopes:}

- Fractionation of ${ }^{15} \mathrm{~N}$ was studied using a nitrogen isotope exchange between a liquid, which is representable as a mixture of liquid $\mathrm{N}_{2} \mathrm{O}_{3}$ and liquid $\mathrm{N}_{2} \mathrm{O}_{4}$, and a gas phase in equilibrium with the liquid. The system was studied at temperatures, $-9^{\circ} \mathrm{C} \sim 20^{\circ} \mathrm{C}$, and under pressures, $1 \sim 4 \mathrm{~atm}$. 
- The effective unit stage separation factor, $\alpha_{\text {off }}$ increases with increasing pressure, e.g., at $15^{\circ} \mathrm{C}$, from 1.006 at $1 \mathrm{~atm}$ to 1.030 at $2.7 \mathrm{~atm}$, and then decreases as the increasing pressure starts to reduce the average oxidation state of nitrogen in the liquid.

- The height equivalent of theoretical plate (HETP) is a linear function of the gas flow rate. The overall exchange rate is diffusion-controlled.

- The HETP of this $\mathrm{NO} / \mathrm{N}_{2} \mathrm{O}_{3}$ system is smaller than that of the Nitrox process, the process based on the exchange between nitric oxide and nitric acid, by an order of magnitude. Thus, the $\mathrm{NO} / \mathrm{N}_{2} \mathrm{O}_{3}$-based plant will have a significantly smaller plant than the Nitrox plant in spite of the smaller $\alpha_{\text {eff }}$ of the $\mathrm{NO} / \mathrm{N}_{2} \mathrm{O}_{3}$ system.

- The product-end refluxing of $\mathrm{NO} / \mathrm{N}_{2} \mathrm{O}_{3}$-based plant will require one-third the amount of sulfur dioxide needed for the product-end refluxing in the Nitrox plant.

- Development of electrode-based hydrophobic catalyst for the hydrogen-isotope exchange between dihydrogen and water was carried out.

- Poly(p-phenylene) film, prepared by electropolymerization of p-terphenyl in acetonitrile and tetraethylammonium tetrafluoroborate is mechanically durable, electrochemically stabie, and as hydrophobic as poly(tetrafluoroethylene). A hydrogen-isotope exchange column can be packed with a conductive packing material, and catalyst metal (e.g., platinum) and poly (p-phenylene) are electro-codeposited on it; an in situ control of hydrophobicity is possible.

- Chemically poisoned platinum surface can be depoisoned easily by subjecting it to an electrode potential excursion, thus making an in situ regeneration of the catalyst bed possible.

- Effective surface area of the edge plane of pyrolytic graphite can be determined from measurements of its electric capacitance using a triangular potential wave of $1 \mathrm{~Hz}$ to $10 \mathrm{~Hz}$.

- The first-order solution of a partial differential equation representing a material balance in a square cascade of close-separation stages derived by Karl Cohen for the total reflux condition was extended to a second-order solution, thus lowering the time limit of applicability of Cohen's solution.

- The transient behavior is not symmetric with respect to change of signs in $\varepsilon(\equiv \alpha-1)$.

- The second-order term is especially important in the domain of inverse isotope effect in which $\varepsilon$ is negative.

- An isotope separation plant based on an inverse isotope effect phenomenon should have a shorter start-up time if all other plant parameters are same.

- It will also be easier to design a product-end refluxer having a small holdup, if the desired isotope enriches in a gas phase. 


\section{Personnel}

\section{Present Afriliation}

\section{Principal Investigator:}

Takanobu Ishida

Professor

State University of New York at Stony Brook

Stony Brook, New York

\section{Scientific Collaborators:}

Jacob Bigeleisen

Francis T. Bonner

Karl Heinzinger

Hidetake Kakihana

Myung W. Lee

Takao Oi

William Spindel
Distinguished Professor Emeritus

State University of New York at Stony Brook

Professor

State University of New York at Stony Brook

Max-Planck Institut für Chemie

Mainz, Germany

Professor Emeritus

Sophia University, Tokyo, Japan

Savannah River Laboratory

E. I. duPont de Nemours

Aiken, South Carolina

Associate Professor

Department of Chemistry

Sophia University, Tokyo, Japan

Senior Consultant

National Academy of Sciences

Washington, D.C.

\section{Postdoctoral Research Associates:}

Motonari Adachi

Madhav Ghate
Assistant Professor

Institute of Atomic Energy

Kyoto University, Japan

Chief, Process \& Project Engineering

Clean Coal Project Division

Morgantown Energy Technology Center

U.S. Department of Energy 
Koichi linuma

Masaaki Musashi

Masato Murata

Anthony Popowicz

Fukuji Yamashita

\section{Graduate Students:}

Lester Borodinsky, Ph.D.

Min Chu, Ph.D.

Lissette Cuevas

Susan Hillenbrand, M.S.

Pat Jayanta, M.S.

Arundhati Kanungo, Ph.D.

Tseng Ven King, Ph.D.

Zvi Kornblum, Ph.D.

Leonard Krebs, M.S.
Assistant Professor

Department of Nuclear Engineering

Tohoku University, Sendai, Japan

Postdoctoral research associate

Department of Chemistry

State University of New York at Stony Brook

Advanced Isotope Separation and Application Section Power Reactor and Nuclear Fuel Development Corp.

Tokai, Japan

Assistant Director of Computing Services

Rockefeller University

Associate Professor

Department of Chemical Engineering

Kanagawa Institute of Technology

Staff Scientist

Keller and Heckman

Washington, D.C.

Postdoctoral Fellow

Department of Chemistry

University of Illinois, Urbana-Champaign

Graduate student

Department of Chemistry

State University of New York

Lecturer

Department of Chemistry

Long Island University, Southhampton, New York

Molecular Biosystems

San Diego, California

Mrs. Michael Prencipe

Patent Examiner

Superconductivity Group

U.S. Patent Office

Professor and Chair

Department of Chemistry

Cooper Uninon, New York 
Peter Lew, Ph.D.

Steven Minick, M.S.

James Pollin, Ph.D.

Michael Prencipe, Ph.D.

Jan Shulman, Ph.D.

Henry Wieck, Ph.D.
Exploitation Technology

U. S. Central Intelligence Agency

Environmental Measurement Laboratory

U.S. Department of Energy, New York

Engineering Manager

Dallas Gas \& Electric, Inc.

Section Head, Abrasives Research Section Colgate Palmolive, New Jersey

Rohm and Haas Co.

Delaware Valley

Division Leader

Chemistry Division

P. A. Consulting Group, New Jersey

\section{Undergraduate Students:}

Mohammad Bahavar

Paul E. Bigeleisen, M.D.

Steven Brandt, Ph.D.

Isaac Cohen, Ph.D.

Jeffrey Feiner, M.D.

David Finston, Ph.D.

Edward Gabalski, M.D.

Mark Gilbert, M.D.

Catherine Harrison, Ph.D.

Richard Hom, M.D./Ph.D.
$\mathrm{Ph} . \mathrm{D}$. Candidate Department of Chemical Engineering Clarkson University

Associate Professor Department of Anesthesiology

University of Minnesota

Director of Pharmaceutical Chemistry Sepractor, Inc.

Marlborough, Massachusetts

Chief, Development Division

Estee Lauder Inc.

Melville, New York

South Orange County Cardiology Group Laguna Hills, California

Associate Professor

Department of Mathematics

New Mexico State University 
William Limm, Ph.D.

Darrell Mayfield, M.S.

Riaz Rouhani, Ph.D.

Ellen Sekreta, Ph.D

David Trauber, M.D.
Food and Drug Administration Washington, D.C.

Geologist

Axem Resources

Denver, Colorado

Postdoctoral Research Associate

Department of Biochemistry

New York Hospital-Cornell Medical Center

Postdoctoral Researcher Associate

Department of Chemistry

University of California, Irvine

Private practice

Brooklyn, New York

Associate Director of Compusting Services

Rockefeller University 


\section{Curriculum Vita}

Takanobu Ishida

B.S. (Chemistry) Kyoto University, 1953; M.S. (Chemistry) Kyoto University, 1955; M.S. (Nuclear Engineering), New York University, 1958; Ph.D. (Nuclear Engineering) Massachusetts Institute of Technology, 1964.

Research Associate, Chemistry Department, Brookhaven National Laboratory, 1964 - '66.

Research Associate, Chemistry Department, Belfer Graduate School of Science, Yeshiva University, 1966 - '68.

Brooklyn College, City Univeristy of New York, Assistant Professor to Professor of Chemistry, 1968 - '79.

University of Rochester, Visiting Associate Professor to Visiting Professor of Chemistry, 1973 - '79.

Professor of Chemistry, State University of New York at Stony Brook, 1979 - .

Visiting Scholar, Max Planck Institut für Chemie, Mainz, 1986.

Research Collaborator, Brookhaven National Laboratory 1972 - .

Member, American Chemical Society

Member, American Physical Society 


\section{[ blank page ]}




\section{Publication}

\section{Papers}

1. Correlation of isotope effects with molecular forces. II. Triatomic molecules", Bigeleisen, J.; I Ishida, T.; Spindel, W., J. Chem. Phys., 1971, 55, 5021.

2. "Isotope chemistry and molecular structure. A spimplified theory of end atom isotope effects", Bigeleisen, J.; Ishida, T., J. Am. Chem. Soc., 1973, 95, 6155.

3. "Vibrational reduced partition function of the Morse orsicllator", Ishida, T., J. Chem. Phys., 1974, 61, 3009.

4. "Isotope chemistry and molecular structure. Total deuterium isotope effects", Bigeleisen, J.; Ishida, T., J. Chem. Phys., 1975, 62, 80.

5. "Isotope chemistry and molecular structure. Carbon and oxygen isotope chemistry", Bigeleisen, J.; Hom, R. C.; Ishida, T., J. Chem. Phys., 1976, 64, 3303.

6. "Isotope chemistry and molecular structure. Deviations from the first rule of the mean", Ishida, T.; Bigeleisen, J., J. Chem. Phys., 1976, 64, 4775.

7. Medium cluster model for vaopr pressure isotope effect", Pollin, J. S.; Ishida, T. , J. Chem. Phys., 1977, 66, 4433.

8. "The influence of molecular orientation on the vapor pressure isotope effect in liquid methanes", Pollin, J. S.; Ishida, T., J. Chem. Phys., 1977, 66, 4442.

9. "Theory of inverse isotope effect", Ishida, T.; Kornblum, Z. C.; Pollin, J. S., Zentralinstitut fuir Isotopen- und Strahlenforschung Mitteilungen, Leipzig, Nr. 5, "Arbeitstagung, Isotope in der Natur, Gera, 22-26 September, 1975", 1976, Akademie der Wissenschaften der DDR, Leipzig, pp. 40-111.

10. "Kinetics of a Square Cascade of Close-Separation Stages under Total Reflux", Wieck, H. J.; Ishida, T., Separation Science, 1977, 12 (No. 6), 587.

11. "Carbon-13 vapor pressure isotope effect in CHF, ", Borodinsky, L.; Wieck, H. J.; Mayfield, D.; Ishida, T., J. Chem. Phys., 1978, 68, 3279.

12. "Approximation of isotopic reduced partition function ratios by the WINIMAX polynomial perturbation method (WIMPER)", Bigeleisen, J.; Lee, M. W.; Ishida,T.; Bigeleisen, P. E., J. Chem. Phys., 1978, 68, 3501.

13. "Isotope effect on zero-point energy shift upon condensation. I. Formulation and application to ethylene, methane, and fluoromethanes", Kormblum, Z. C.; Ishida,T., J. Chem. Phys., 1978, 69, 1814. 
14. "Correlation of the isotope chemistry of hydrogen, carbon, and oxygen with molecular forces by the WIMPER(2) method", Bigeleisen, J.; Ishida, T.; Lee,M.W., J. Chem. Phys., 1981, 74, 1799.

15. "Studies in the vibrational spectroscopy of sodium trioxodinitrate", Bonner, F. T.; Akhtar, M. J.; King, T. V.; Chen, L. H.; Ishida, T., J. Phys. Chem., 1981, 85, 4051.

16. "Ab initio molecular orbital study of dimer $\mathrm{CHF}_{3}$ ", Popowicz, A. ; Ishida, T., Chem. Phys. Lett., $1981,83,520$.

17. "Vapor pressure isotope effects in liquid fluoroform", Popowicz, A.; Oi, T.; Shulman, J.; Ishida, T., J. Chem. Phys., 1982, 76, 3732.

18. "Synthesis and purification of methyl- ${ }^{13} \mathrm{C}$ fluoride", Cohen, I.; Shulman, J.; Popowicz, A.; Ishida, T. J. Labelled Cmpnd. Radiopharm., 1982, 19, 631.

19. "Correlation of zero-point energy with molecular structure and molecular forces. I. Development of approximation", Oi, T.; Ishida, T., J. Phys. Chem., 1983, 87, 1067.

20. "Ab initio molecular orbital calculation on clusters of methyl fluoride", Oi, T.; Sekreta, E.; Ishida, T., J. Phys. Chem., 1983, 87, 2323.

21. "Vapor pressure isotope effects in liquid methyl fluoride", Oi, T.; Shulman, J.; Popowicz, A.; Ishida, T., J. Phys. Chem., 1983, 87, 3153.

22. "Correlation of zero-point energy with molecular structure and molecular forces. II. Approximation for n-paraffins", Oi, T.; Ishida, T., J. Phys. Chem., 1984, 88, 1507.

23. "Correlation of zero-point energy with molecular structure and molecular forces. III. Approximation for H/D isotope shifts and linear frequency sum rule", Oi, T.; Ishida, T., J. Phys. Chem., 1984, 88, 2057.

24. "Nitrogen-15 fractionation by countercurrent exchange between liquid $\mathrm{N}_{2} \mathrm{O}_{3}-\mathrm{N}_{2} \mathrm{O}_{4}$ mixture and their vapor phases under pressured conditions", Prencipe, M.; Spindel, W.; Ishida, T., Sep. Sci. Tech., 1985, 20, 489.

25. "Addivitity of vibrational zero-point energy", Oi, T. ; Popowicz, A. ; Ishida, T., J. Phys. Chem., 1986, 90, 3090.

26. "Valence coordinate contributions to zero-point energy shifts due to hydrogen isotope substitution", Oi, T. ; Ishida, T., Bull. Chem. Soc. Jpn., 1986, 59, 373.

27. "Orthogonal approximation and classification of molecules for zero-point energy shifts due to H/D isotope substitutions", Oi, T.; Ishida, T., Bull. Chem. Soc. Jpn., 1986, 59, 3669.

28. "Vapor pressure isotope effects in liquid methylene difluoride", Kanungo, A. ; Oi, T. ; Popowicz, A.; Ishida, T., J. Pkys. Chem., 1987, 91, 4198.

29. "Zero-point energy shifts in hydrides of the elements upon H/D isotope substitutions and their periodicity", Oi, T.; Aikawa, M.; Kidetake, H.; Ishida, T., Z. Naturforsch. 1988, 43a, 511.

30. "Molecular dynamics study of a lithium ion in ammonia", Hannongbua, S. V. ; Ishida, T.; Spohr, E.; Heinzinger, K., Z. Naturforsch., 1988, 43a, 572. 
31. "Vapor pressure isotope effects in liquid and solid ammonia", King, T. V. ; Oi, T.; Popowicz, A.; Heinzinger, K.; Ishida, T., Z. Naturforsch., 1989, 44a, 359.

32. "Isotope Separation", Spindel, W. ; Ishida, T. in "Encyclopedia of Physics", Lerner, R. G.; Trigg, G. L., Eds., 1991, VCH Publishers, New York.

33. "Isotope Separation", Spindel, W.; Ishida, T., J. Chem. Educ., 1991, 68, 312.

34. "Molecular forces, isotope effects, and isotope separation", Ishida, T. in "Isotope Separation and Chemical Exchange Uranium Enrichment", Bull. Res. Lab. Nucl. React., Special Issue No. 1, 1992, Fujii, Y.; Ishida, T.; Takeuchi, K., Eds., Tokyo Institute of Technology, Tokyo.

35. "Correlation of vihrational zero-point energy shifts of hydrides upon H/D isotope substitutions with electronegativity", Oi, T.; Ishida, T. [Submitted to J. Chem. Soc. Jpn.]

\section{Editor/Co-Editor}

1. "Isotope Chemistry: Festschrift für Jacob Bigeleisen", Z. Naturforsch. 1989, 44a, Ishida, T.; Saunders, M.; Van Hook, W. A.; Wolfsberg, M., Eds.

2. "Isotope Separation and Chemical Exchange Uranium Enrichment", Bull. Res. Lab. Nucl. React., Special Issue No. 1, 1992, Fujii, Y; Ishida, T; Takeuchi,K., Eds,. Tokyo Institute of Technology.

\section{Manuscripts in Preparation}

201. "Influence of surface oxides on contact angle of water on polycrystalline platinum", Krebs, L. C.; Ishida, T.

202. "Electrochemical modification of polycrystalline platinum surface by poly(p-phenylene)", Krebs, L. C.; Ishida, T.

203. "Effective surface area and capacitance of edge plane of pyrolytic graphite in acetonitrile solutions", Minick, S. K.; Ishida, T. 


\section{Introduction}

General objective of this project has been investigation of isotope effect phenomena and development of fractionation methods for stable isotopes. This part of present report consists of the following four sections:

Section II. Theoretical correlation of isotope effects with molecular forces and molecular structure.

Section III. Theoretical correlation of zero-point energy and its isotope effects with molecular forces and molecular structure.

Section IV. Experimental and theoretical studies of vapor pressure isotope effects.

Section V. Experimental and theoretical studies of fractionation of stable isotopes.

Section V-B deals with studies of hydrophobic catalysts for the hydrogen isotope exchange between dihydrogen and water. Results of these studies will be reported in some detail since they have not yet been published.

Following convention will be used for the reference numbers: The numbers below 100 refer to the publications that have resulted from the Department of Energy support, the numbers in 10\%'s are other publications, and those in 200's refer to our studies performed under this support but the results of which have not been published. List of the first category is found under Publications on pages $x v-x v i i$, one for the second category under References on pages 77-79, and one for the third category under Manuscripts in Preparation on page xvii.

Isotope effects look their simplest when viewed from the perspective point of the reduced partition function ration (RPFR). ${ }^{(101)}$ The $R P F R$ is the ratio of the reduced partition functions $\left(Q_{r}\right.$ and $\left.Q_{r}\right)$ for a pair of isotopic molecules

$$
\frac{s}{s^{\prime}} f=\frac{Q_{r}}{Q_{r}^{\prime}}=\frac{\left(\frac{Q}{Q^{\prime}}\right)_{e}}{\left(\frac{Q}{Q^{\prime}}\right)_{d}}
$$

In Eq. (1), $Q$ and $Q^{\prime}$ are the partition functions of the heavier and lighter isotopic molecules in the isotopic pair under consideration, respectively; the subscripts $\mathrm{gm}, \mathrm{cl}$, and $\mathrm{r}$ refer to the quantum mechanical, classical, and reduced partition functions, respectively; $s$ and $s$ are the symmetry numbers of the heavier and lighter isotopic molecules.

The RPFR is a property of a particular substitution at a given atomic position in a given substance. Value of RPFR directly depends on the molecular environment surrounding the isotopically substituted 
atom. It is also indirectly influenced by other environmental factors such as the physical states, temperature, pressure, density, and intermolecular forces.

Both the equilibrium and kinetic isotope effects on all physicochemical properties are expressible as a ratio of, usually, two RPFRs. The great utility of isotope effect (IE) studies lie in the fact that, to the extent the Born Cppenheimer approximation holds, many structural and counting factors of partition functions cancel for the RPFR between the pair of isotopic molecules [cf: Eq. (1)]. Additional factors cancel for the ratio of two RPFRs. Two examples of such ratio of RPFRs will be given below. These examples represent a large majority of the isotope effects that have been studied in this project.

The equilibrium constant for an isotope exchange equilibrium,

$$
\mathrm{AX}+\mathrm{BX}^{\prime} \rightleftharpoons \mathrm{AX}+\mathrm{BX}^{\prime} \text {. }
$$

is given by

$$
K=\frac{\left[Q^{\prime}\left(\Delta X^{\prime}\right) / Q(A x)\right]_{e m}}{\left[Q^{\prime}\left(B X^{\prime}\right) / Q(B X)\right]_{m}}=\frac{\left[\left(s / s^{\prime}\right) f\right]_{\Delta x}}{\left[\left(s / s^{\prime}\right) f\right]_{\Delta x}} \cdot \frac{\left(\frac{t^{\prime}}{s}\right)_{\Delta x}}{\left(\frac{1}{s}\right)_{\Delta x}} .
$$

Generally, A and B in Eq. (2) are polyatomic moieties of respective molecules. The isotope fractionation factor, $\alpha$, due to Reaction (2) is expressible as

$$
\alpha=\frac{\left[\left(s / s^{\prime}\right) f\right]_{B x}}{\left[\left(s^{\prime}\right) f\right]_{d r}}
$$

In both Eqs. (3) and (4) the mass- and moment of inertia factors contained in the classical partition functions cancel out for the balanced chemical equation such as Eq. (2). (101) For an isotope exchange equilibrium in the gas phase, the only remaining factors of $f_{B x}$ and $f_{A X}$ are those due to the internal vibrations of the isotopic molecular pairs, $\mathrm{AX} / \mathrm{AX}$ and $\mathrm{BX} / \mathrm{BX}$. For an equilibrium in a condensed phase, $f_{B X}$ and $f_{A X}$ also contain contributions from intermolecular intereactions. The high-temperature limit of $K$ is the classical equilibrium contant, $K_{c 1}$,

$$
K_{c l}=\frac{\left(s^{\prime} / s\right)_{a x}}{\left(s^{\prime} / s\right)_{d x}}
$$

So that

$$
\alpha=\frac{K}{K_{d}}
$$

Both $K$ and $\alpha$ are functions of temperature. However, the ratio of $\alpha$ and $K$ is always a constant, which only depends on the symmetry numbers.

The second example is the isotopic vapor pressure ratio, which is expressible as

$$
\ln \frac{P^{\prime}}{P}=\left[\ln \frac{f_{c}}{f_{t}}\right] \cdot[\text { correction factors }],
$$


where $P^{\prime}$ and $P$ are the vapor pressures of the lighter and heavier isotopic substances, $f_{c} / f_{b}$ is the ratio of the RPFR of the condensed phase to that of the vapor phase, and the "correction factors" correct for relatively small corrections such as the gas imperfection and liquid molar volume effects.

As a result of the cancellations of the otherwise dominant factors when the ratio of the ratios is evaluated, only those intra- and intermolecular interactions that are affected by the isotopic substitution manifest themselves in the isotope effects. Isotopic substitutions at strategic locations in a molecular susbtance is thus an extremely sensitive probe for elucidating for the molecular structures and molecular forces. 
[ blank page ] 


\section{Correlation of Isotope Effects with Molecular Forces and Structure}

The isotopic reduced partition function ratio (RPFR) of an ideal gas is

$$
\ln \left(s / s^{\prime}\right) f=\sum_{i=1}^{f} \ln \frac{u_{i}}{u_{i}^{\prime}} \frac{e^{-u_{1} / 2} /\left(1-e^{-u_{1}}\right)}{e^{-u_{1}^{\prime} / 2} /\left(1-e^{-u_{1}^{\prime}}\right)}
$$

where

$$
u_{i}=\frac{h c v_{i}}{k T}
$$

and $f=3 N-6$ or $3 N-5$. A self-consistent set of vibrational frequencies $v_{i}^{\prime}$ (for the lighter isotopic molecule) and $v_{i}$ (for the heavier molecule) are obtained from vibrational eigenvalues $\lambda_{j}^{\prime}$ and $\lambda_{1}$;

$$
\lambda_{i}^{\prime}=4 \pi^{2} v_{i}^{\prime 2} \quad \text { and } \quad \lambda_{1}=4 \pi^{2} v_{i}^{2}
$$

as solutions of

$$
\left|\mathbf{H}^{\prime}-\lambda \mathbf{I}\right|=0 \text { and }|\mathbf{H}-\lambda I|=0
$$

where $I$ is a unit matrix,

and

$$
\mathbf{H}^{\prime}=\left\{h_{\mathrm{ij}}^{\prime}\right\} \text { and } \mathbf{H}=\left\{\mathrm{h}_{\mathrm{ij}}\right\} \text {, }
$$

$$
\mathbf{H}^{\prime}=\mathbf{G}^{\prime} \mathbf{F} \text { and } \mathbf{H}=\mathbf{G} \mathbf{F} \text {. }
$$

In Eq. (13), $\mathbf{G}$ is Wilson's G-matrix, and $\mathbf{F}$ is the corresponding matrix of harmonic force constants:

$$
\mathbf{G}^{\prime}=\left\{\mathbf{g}_{i j}^{\prime}\right\} \quad, \mathbf{G}=\left\{g_{i j}\right\} \quad, \text { and } \mathbf{F}=\left\{f_{i j}\right\} .
$$

Except for the molecules which possess a very high symmetry, Eq. (11) requires a numerical solution involving an algorithm which consists of a simultaneous diagonalization of two square, positive definite matrices, $\mathbf{F}$ and $\mathbf{G}$. Such a procedure represents a black-box, as far as our physical understanding of isotope effects in terms of the molecular forces and structures is concerned.. We were in need of an analytic expression of $\ln \left(\mathrm{s} / \mathrm{s}^{\prime}\right) f$ in explicit terms of the elements of $F$ and $G$ matrices for such elucidations when this project was started. 


\section{II-A. Development of Theoretical Tools}

The wanted tool is an approximation of an acceptable precision for the function, $\ln \left(\mathrm{s} / \mathrm{s}^{\prime}\right) \mathrm{f}$, of Eq. (8), directly expressed in terms of $f_{i j}$ 's, $g_{i j}$ 's and the temperature. The first attempt toward this goal was Bigeleisen's Taylor series expansion ${ }^{(103)}$ :

$$
\ln \frac{s}{s^{\prime}} f=\sum_{m=1}^{\infty} \frac{(-1)^{m+1} B_{2 m-1}}{2 m(2 m) !} \sum_{i=1}^{f} \delta u_{i}^{2} \quad\left[\text { All } u \text { and } u^{\prime}<2 \pi\right],
$$

where $B_{k}$ are Bernoulli numbers $\left(B_{1}=1 / 6, B_{3}=1 / 30, B_{3}=1 / 42\right.$, etc.), and

$$
\delta u_{i}^{2 m}=u_{i}^{2 m}-u_{i}^{2 m} \text {. }
$$

This infinite series converges only when the largest of all $u_{i}$ 's is smaller than $2 \pi$. This means that, for instance, at 300K all vibrational frequencies must be lower than $1310 \mathrm{~cm}^{-1}$. The series is thus useful only at considerably low temperatures, e.g., $\mathrm{T}<200 \mathrm{~K}$.

We have developed, in collaboration with the Bigeleisen group, several new approximations which eliminated the severe limitation on frequencies and improved the precision of the approxim ation in several steps. ${ }^{(2,7,12,14,105,106)}$ They are all based on the principles of orthogonal polynomial approximation. In the following, these methods will be summarized in two groups, that is, the methods based on a direct expansion of $\ln \left(\mathbf{s} / \mathbf{s}^{\prime}\right) \mathrm{f}$, and a second group of methods which utilize the diagonal matrices, $\mathbf{G}_{\mathrm{o}}=\left\{\mathrm{g}_{\mathrm{ij}} \delta_{\mathrm{ij}}\right\}$ and $F_{0} \equiv\left\{f_{i j} \delta_{i j}\right\}\left[\delta_{i j}=\right.$ Kronecker delta], as a zero-th order approximation.

\section{II-A-1. Direct Orthogonal Polynomial Approximations}

In an orthogonal expansion, a function $f(x)$ is exprressed as a series of terms of mutually orthogonal functions. An orthogonal polynomial oscillates within a region of orthogonality. The error of a truncated series approximation of $f(x)$ thus oscillates in the domain in which the orthogonality is defined, rather than the error gradually increasing with the $v$, riable, $x$, moving away from the center of expansion.

The RPFR of Eq. (8) can be written as

$$
\ln \frac{s}{s^{\prime}} f=\sum_{i}\left[\ln b\left(u_{i}^{\prime}\right)-\ln b\left(u_{i}\right)\right],
$$

where

$$
\begin{aligned}
\ln b(u) & =-\ln u+\frac{u}{2}+\ln \left(1-e^{-u}\right) \\
& =\sum_{k=1}^{\infty} \ln \left[1+\left(\frac{u}{2 \pi k}\right)^{2}\right]
\end{aligned}
$$


An application of Lanczos' $\tau$-method ${ }^{(104)}$ on the function, $\ln (1+x)$, leads to the following orthogonal polynomial approximation ${ }^{(105,106)}$ for the RPFR:

$$
\ln \frac{s}{s^{\prime}} f=\sum_{m=1}^{n} W_{m}\left(n, u_{\max }^{\prime}, L\right) A_{m} \sum_{i=1}^{f} \delta u_{i}^{2 m},
$$

where $\mathrm{n}$ is the order of the expansion, $\mathrm{f}$ the degree of freedom, $\mathrm{L}$ an integer parameter for the expansion used to isolate several lowest-order terms, and

$$
A_{m}=\frac{(-1)^{2 m+1} B_{2 m-1}}{2 n(2 m) !}
$$

and

$$
W_{m}\left(n, u_{\max }^{\prime}, L\right)=T\left(n, m, R_{L+1}\right)+\frac{1}{z(m)} \sum_{k=1}^{L} \frac{T\left(n, m, R_{k}\right)-T\left(n, m, R_{L+1}\right)}{k^{2 m}}
$$

in which

$$
T(n, m, R)=\frac{\sum_{p=m}^{n} \frac{(-1) C_{p}^{n}}{N}}{\sum_{p=0}^{n} \frac{(-1) C_{p}^{n}}{p}},
$$

and

$$
R_{k}=\frac{1}{k^{2}}\left(\frac{u_{\max }^{\prime}}{2 \pi}\right)^{2} \quad[k=L+1, L+2, \ldots] .
$$

In Eqs. (21) and (22), $z(m)$ is the Riemann zeta function, and $C_{n}^{p}$ is the coefficient of the orthogonal polynomial $P_{n}(x)$ used for the approximation,

$$
P_{n}(x)=\sum_{m=0}^{n} C_{n}^{m} x^{m}
$$

The usefulness of the orthogonal expansion, Eq. (19), lies in the fact that it converges for any frequency at any temperature and that, as in the Bigeleisen series of Eq. (15), the term $\Sigma_{i} \delta u_{i}^{2 m}$ is expressible in terms of the trace of the $m$-th power of the product of $F$ and $G$-matrices:

$$
\begin{aligned}
\sum_{i=1}^{f} \delta u_{i}^{2 m} & =\left(\frac{\hbar c}{k T}\right)^{2 m} \sum_{i=1}^{f} \delta \lambda_{i}^{m} \\
& =\left(\frac{\hbar c}{k T}\right)^{2 m}\left\{\operatorname{Tr}\left[\left(\mathrm{FG}^{\prime}\right)^{m}\right]-\operatorname{Tr}\left[(\mathrm{FG})^{m}\right]\right\} \\
& =\left(\frac{\hbar c}{k T}\right)^{2 m}\left[\delta \operatorname{Tr}\left(\mathrm{H}^{m}\right)\right]
\end{aligned}
$$

Through Eq. (25), in (s/s)f and thus all isotope effects can be directly correlated to the molecular forces via the F-matrix and to the molecular geometry via the G-matrix. For instance, for $m=1$ and 2 , we have 


$$
\Sigma_{i} \delta \lambda_{i}=\Sigma_{i} \delta\left(g_{i i}\right) f_{i i}+2 \Sigma \Sigma_{i<j} \varepsilon\left(g_{i j}\right) f_{i j},
$$

and

$$
\begin{aligned}
& \Sigma_{i} \delta \lambda_{i}^{2}=\Sigma_{i} \delta\left(g_{i i}^{2}\right) f_{i i}^{2}+\Sigma \Sigma_{i \sim j} \delta\left(g_{i i} g_{j j}\right) f_{i j}^{2} \\
& +4 \Sigma \Sigma_{i \neq j} \delta\left(g_{i i} g_{i j}\right) f_{i i} f_{i j} \\
& +2 \Sigma \Sigma_{i * j} \delta\left(g_{i j}^{2}\right)\left(f_{i i} f_{j j}+f_{i j}^{2}\right) \\
& +2 \Sigma \Sigma \Sigma_{i \neq j \neq k} \delta\left(g_{i i} g_{j k}\right) f_{i j} f_{i k} \\
& +2 \Sigma \Sigma \Sigma_{i \neq j \neq k} \delta\left(g_{i j} g_{i k}\right)\left(f_{i i} f_{j k}+f_{i j} f_{i k}\right) \\
& +\sum \Sigma \Sigma \Sigma_{i \neq j \neq k \neq 1} \delta\left(g_{i j} g_{k l}\right)\left(f_{i l} f_{j k}+f_{i k} f_{j l}\right) \text {. }
\end{aligned}
$$

The approximation for $\ln b(u)$ that corresponds to Eq.(19) for $\ln \left(s / s^{\prime}\right) f$ is

$$
\ln b(u)=\sum_{m=1}^{n} W_{m}\left(n, u_{\max }^{\prime}, L\right) A_{m} u^{2 m} \quad .
$$

When the shifted Chebyshev polynomials of the first kind is used, examples of the errors that Eq. (28) yields are, with $\mathrm{L}=0$ :

$$
\begin{array}{rllll}
6.6 \%(n=2), & 0.12 \%(n=3), & \text { and } 0.0033 \%(n=4) & \text { at } u=6 ; \\
7.1 \%(n=2), & 2.0 \%(n=3), & \text { and } 1.6 \% \quad(n=4) & \text { at } u=16 ; \\
19.6 \%(n=2), & 7.2 \% \quad(n=3), & \text { and } 1.7 \% \quad(n=4) & \text { at } u=18 .
\end{array}
$$

A general improvement in the approximation was achieved by constructing an optional set of Jacobi polynomials for this mathernatical problem. ${ }^{(100)}$ The Jacobi polynomials, $P_{\mathbf{a}}{ }^{(1.8)}(x)$, are a class of Gaussian hypergeometric functions charaterized by the order, $n$, of the polynomial and two parametric constants, $\gamma$ and $\delta$. The optimal set of Jacobi polynomia's and Eq. (28) yielded a three- to ten-fold improvements in the precision. For instance, with $\mathrm{L}=5$;

$$
\begin{array}{llll}
0.62 \%(n=2), & 0.083 \%(n=3), & \text { and } 0.011 \%(n=4) & \text { at } u=6 ; \\
2.8 \%(n=2), & 2.8 \%(n=3), & \text { and } 0.66 \%(n=4) & \text { at } u=12 ; \\
5.7 \%(n=2), & 2.1 \%(n=3), & \text { and } 0.82 \%(n=4) & \text { at } u=18 .
\end{array}
$$

\section{I-A-2. Orthogonal Expansion Methods with Diagonal Approximation}

The precision of approximation of the orthogonal expansion methods was further improved without losing the expliciteness of Eq. (19) and the contact with the physical significance ${ }^{(12,14)}$ by recognizing a fact that the diagonal part of F-matrix,

$$
F_{0} \equiv\left\{f_{i j} \delta_{i j}\right\},
$$

and the diagonal part of G-matrix,

$$
\mathbf{G}_{0}=\left\{\boldsymbol{g}_{\mathrm{ij}} \boldsymbol{\delta}_{\mathrm{ij}}\right\},
$$


can be used as the zero-th order approximation for the actual matrices, $F=\left\{f_{i j}\right\}$ and $G \equiv\left\{g_{i j}\right\}$, respectively. This fact was used by Wolfsberg in his perturbation approximation theory ${ }^{(11)}$ for RPFR. Each element of the zero-th order approximation represents the contribution of each valence coordinate regarded to be an independent harmonic oscillator, and the higher order terms are contributions of inter-coordinate interactions. The RPFR for the hypothetical polyatomic molecule corresponding to the $\left\{F_{0}, G_{0}\right\}$ set is

$$
\begin{aligned}
\ln \frac{s}{s^{\prime}} f & =\sum_{m=1}^{\infty} W_{m} A_{m}\left(\frac{h c}{k T}\right)^{2 m} \sum_{i=1}^{f} \lambda_{i, 0}^{m} \\
& =\sum_{m=1}^{\infty} W_{m} A_{m} \operatorname{Tr}\left(\mathbf{H}_{0}^{m}\right)
\end{aligned}
$$

where

$$
\operatorname{Tr}\left(\mathbf{H}_{0}^{m}\right)=\sum_{i=1}^{f} f_{i i}^{m} g_{i i}^{m} \quad .
$$

Since the magnitudes of the diagonal elements, $f_{i}$ and $g_{i} i$, are much greater than those of the off-diagonal elements, in $\left(s^{\prime} / s^{\prime}\right) f_{0}$ is a good zero-th order approximation for the real in $\left(s / s^{\prime}\right) f$. Then, the orthogonal expansion methods described in the preceding Section could be used to approximate the remainder. Thus,

$$
\begin{aligned}
\ln \frac{s}{s^{\prime}} f & =\ln \frac{s}{s^{\prime}} f_{0}+\left(\ln \frac{s}{s^{\prime}} f-\ln \frac{s}{s^{\prime}} f_{0}\right) \\
& =\ln \frac{s}{s^{\prime}} f_{0}+\Sigma_{m} W_{m} A_{m}\left(\frac{h c}{k T}\right)^{2 m} \delta \Delta_{0} \operatorname{Tr}\left(\mathbf{H}^{m}\right),
\end{aligned}
$$

where

$$
\begin{aligned}
\delta \Delta_{0} \operatorname{Tr}\left(\mathbf{H}^{m}\right) & =\delta\left[\operatorname{Tr}\left(\mathbf{H}^{m}\right)-\operatorname{Tr}\left(\mathbf{H}_{0}^{m}\right)\right] \\
& =\left[\operatorname{Tr}\left(\mathbf{H}^{\prime m}\right)-\operatorname{Tr}\left(\mathbf{H}_{0}^{\prime m}\right)\right]-\left[\operatorname{Tr}\left(\mathbf{H}^{m}\right)-\operatorname{Tr}\left(\mathbf{H}_{0}^{m}\right)\right]
\end{aligned}
$$

in which the prime refers to the lighter of the two isotopic molecule, as usual. All terms contained in the last expression of Eq. (34b) are generally much smaller than the terms $f_{i i} g_{i}$ of $\ln \left(s / s^{\prime}\right) f_{0}$. For $m=1$ and $m=2$, Eq. (34) become

$$
\delta \Delta_{0} \operatorname{Tr}(\mathbf{H})=\sum_{i=j} \sum_{j} \delta\left(g_{i j}\right) f_{i j}
$$

and

$$
\begin{aligned}
\delta \Delta_{0} \operatorname{Tr}\left(\mathbf{H}^{2}\right) & =\sum_{i=} \sum_{j}\left[4 \delta\left(g_{i i} g_{i j}\right) f_{i i} f_{i j}+\delta\left(g_{i j}^{2}\right)\left(f_{i j}^{2}+f_{i i} f_{j j}\right)+\delta\left(g_{i i} g_{j j}\right) f_{i j}\right] \\
& \left.+\sum_{i=k} \sum_{j=}\left[2 \delta\left(g_{i i} g_{j k}\right) f_{i j} f_{i k}+2 \delta g_{i j} g_{i k}\right)\left(f_{i i} f_{j k}+f_{i j} f_{i k}\right)\right] \\
& +\sum_{i=k} \sum_{j=k} \sum_{k=1} \delta\left(g_{i k} g_{j l}\right) f_{i l} f_{j k} .
\end{aligned}
$$


In a variation of the diagonal-element approximation, Eq. (33), the zero-th order term $\ln \left(s / s^{\prime}\right) f_{0}$, is replaced by $\ln \left(s / s^{\prime}\right) f_{d}$, which is evaluated by using the diagonal terms of $\mathbf{H}_{d}$, a matrix obtained by setting all off-diagonal term of the product of $\mathbf{G}$ and $\mathbf{F}$ matrices to zero:

$$
\mathbf{H}_{\boldsymbol{d}} \mathbf{z}\left\{\boldsymbol{h}_{i j} \boldsymbol{\delta}_{i j}\right\},
$$

where

$$
h_{i j}=\sum_{k} f_{i k} g_{k j},
$$

so that the $i-$ th eigenvalue of $\left|H_{d}-\lambda I\right|=0$ is

$$
\lambda_{i, d}=h_{i i}=\sum_{k} f_{i k} g_{k i} \text {. }
$$

Thus,

$$
\ln \frac{s}{s^{\prime}} f=\ln \frac{s}{s^{\prime}} f_{d}+\sum_{m} W_{m} A_{m}\left(\frac{h c}{k T}\right)^{2 m} \delta \Delta_{d} \operatorname{Tr}\left(\mathbf{H}^{m}\right)
$$

where

$$
\begin{aligned}
\delta \Delta_{d} \operatorname{Tr}\left(\mathbf{H}^{m}\right) & =\delta\left[\operatorname{Tr}\left(\mathbf{H}^{m}\right)-\operatorname{Tr}\left(\mathbf{H}_{d}^{m}\right)\right] \\
& =\left[\operatorname{Tr}\left(\mathbf{H}^{\prime m}\right)-\operatorname{Tr}\left(\mathbf{H}_{d}^{\prime m}\right)\right]-\left[\operatorname{Tr}\left(\mathbf{H}^{m}\right)-\operatorname{Tr}\left(\mathbf{H}_{d}^{m}\right)\right] .
\end{aligned}
$$

An advantage of the in $\left(s / s^{\prime}\right) f_{d}$ approximation over the in $\left(s / s^{\prime}\right) f_{0}$ approximation is that the zero-th order approximation in the former takes into account some of the effects of the first-order interactions terms. For $m=1$ and $m=2$, Eq. (41) becomes

$$
\begin{aligned}
\delta \Delta_{d} \operatorname{Tr}(\mathbf{H}) & =0, \\
\Delta_{d} \operatorname{Tr}\left(\mathbf{H}^{2}\right) & =\sum_{i=} \sum_{j}\left[2 \delta\left(g_{i i} g_{i j}\right) f_{i i} f_{i j}+\delta\left(g_{i j}^{2}\right) f_{i i} f_{j j}+\delta\left(g_{i i} g_{j j}\right) g_{i j}^{2}\right] \\
& +\sum_{i=} \sum_{j=k}\left[2 \delta\left(g_{i i} g_{j k}\right) f_{i j} f_{i k}+\delta\left(g_{i j} g_{i k}\right)\left(2 f_{i i} f_{j k}+f_{i j} f_{i k}\right)\right] \\
& +\sum_{i=j k k} \sum_{k=1} \delta\left(g_{i k} g_{j l}\right) f_{i l} f_{j k}
\end{aligned}
$$

Although one is able to set a general mathematical error bound for the approximations for $\ln b(u)$, the same is not toue for the approximations of $\ln \left(s^{\prime} / s^{\prime}\right) f$. This is because $\ln \left(s / s^{\prime}\right) f$ depends on particular distributions of the vibrational frequencies of molecules and their isotope shifts. In spite of these limitations, the precisions of the approximations are sufficiently high to have allowed us to find useful correlations of isotope effects with various structural features of real molecules. These results will be summarized in next section. 


\section{I-B. Correlation Studies}

In terms of the orthogonal polynomial approximation, effects of molecular geometry on the isotope effect phenomena manifest themselves through the various elements of Wilson's G-matrix. ${ }^{(100)}$ Hydrogen atoms in hydrogenous molecules are terminal atoms except in hydrogen-bonding structures. An isotope substitution at the positions of these hydrogen atoms and other end-atom substitutions lead to a type of isotope effects which are simple to analyze, mainly due to relatively simple differences in the G-matrices of these isotopic molecules. (14,6, 12, 14, 10s,107) The substitutions at positions other than an end position, on the other hand, cause far-reaching effects down several chemical bonds and thus generally require a more careful attention. ${ }^{(5,12,14,34,105,106)}$

\section{II-B-1. End-Atom Isotope Effects}

In Figure 1, atoms 1, 4 and 5 are examples of endatom, and atoms 2 and 3 are examples of non-end atom. Elements of G-matrix are generally given by ${ }^{(108)}$

$g_{i j}=\sum_{\alpha}^{\text {All atoms }} \mu_{\alpha} \vec{s}_{i \alpha} \bullet \vec{s}_{j \alpha}$

where $\mu_{\alpha}$ is the reciprocal mass of atom $\alpha$, and $\vec{s}_{i \alpha}$

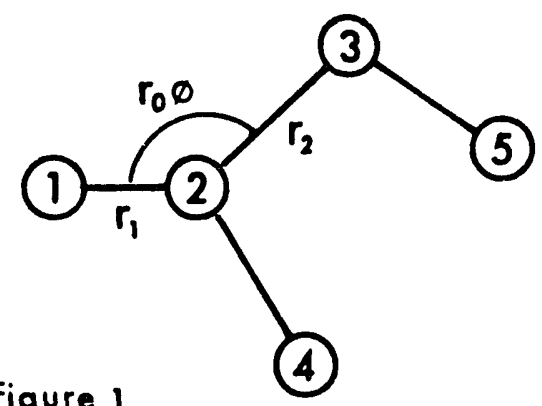
is Wilson's \&-vector for the coordinate $i$ and atom $\alpha$.

Referring to Figure 2, we have

$$
\vec{s}_{n, 1}=-\vec{e}_{12}, \vec{s}_{n 1,2}=\vec{e}_{12}, \quad \vec{s}_{n, 3}=\overrightarrow{0},
$$

and

$$
\vec{s}_{2,1}=\overrightarrow{0}, \vec{s}_{2,2}=\vec{e}_{32}, \vec{s}_{2,3}=-\vec{e}_{32} .
$$

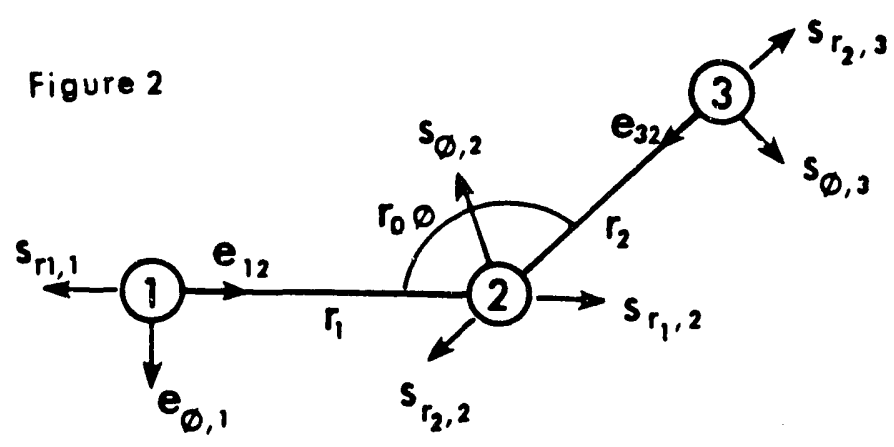


where $\vec{e}_{\alpha \beta}$ is a unit vector directed from atom $\alpha$ to atom $\beta$. For the bending coordinate $\phi$, the vectors $s_{\phi, 1}$ and $s_{8,}$, are perpendicular to the unit vectors $e_{12}$ and $e_{31}$, respectively, but the vector $s_{8,2}$ is not perpendicular to either bond, 1-2 or 3-2, unless the equilibrium bond angle is $90^{\circ}$.

Thus, the diagonal elements are;

$$
g_{n}=\mu_{1}+\mu_{2}, \quad g_{n}=\mu_{2}+\mu_{3}, \quad g_{1}=\mu_{1} \rho_{1}+\mu_{2} p_{2}+\mu_{3} \rho_{3}
$$

where

$$
\rho_{1}=\left(r_{0} / r_{12}\right)^{2}, \quad \rho_{3}=\left(r_{0} / r_{23}\right)^{2}, \quad \rho_{2}=r_{0}^{2}\left(1 / r_{12}{ }^{2}+1 / r_{32}{ }^{2}-2 \cos \phi_{0} / r_{12} r_{23}\right)
$$

and the off-diagonal elements are;

$$
\begin{aligned}
& g_{n 1, \phi}=\left(s_{n 1,2} \cdot s_{0,2}\right) \mu_{2}=-\mu_{2}\left(r_{0} / r_{12}\right) \sin \phi_{0} \\
& g_{n, \downarrow}=\left(s_{n, 2} \cdot s_{0,2}\right) \mu_{2}=-\mu_{2}\left(r_{0} / r_{23}\right) \sin \phi_{0} \\
& g_{n 1, n}=\left(s_{n 1,2} \cdot s_{n, 2}\right) \mu_{2}=-\mu_{2} \cos \phi_{0}
\end{aligned}
$$

We note that the kinetic energy interaction between a bond-stretching motion and an angle-bending motion depends only on the mass of the central atom such as atom 2 in Figure 2 and not on the terminal atoms such as atoms 1 and 3 in Figure 2. This is due to the fact that the vector $8_{8,1}$ is perpendicular to the vector $e_{12}$. The isotopic difference $\delta g_{11,}$ for an isotope substitution at Position 1 vanishes. In general, $\delta g_{i j}=0$ between a stretching coordinate and a bending coordinate for an end-atom substitution. Furthermore, all off-diagonal elements $g_{i j}$ between a bond-stretching motion and a torsion of an adjacent bond vanish due to the similar perpendicularity of the corresponding \&-vectors.

We studied end-atom isotope effects in $\mathrm{CO}_{2}^{(1)}$ and, $\mathrm{H}_{2} \mathrm{O}, \mathrm{CH}_{2} \mathrm{O}, \mathrm{CH}_{4}, \mathrm{C}_{2} \mathrm{H}_{4}, \mathrm{C}_{2} \mathrm{H}_{6}$ and $\mathrm{C}_{6} \mathrm{H}_{6}{ }^{(4,12,14)}$ in the light of the orthogonal polynomial approximations of RPFR. For an end-atom substitution the only intercoordinate kinetic energy couplings $\left(g_{i j}\right)$ that contribute to $\mathrm{ln}\left(\mathrm{s} / \mathrm{s}^{\prime}\right) f$ are the adjacent bend-bend interactions such as those among the three $\mathrm{HCH}$ angle bends sharing a $\mathrm{C}-\mathrm{H}$ bond in methane. All other $g_{i j}$ 's ( $\left.i \neq j\right)$ vanish for the end-atom isotope effect. Furthermore, the contributions of interactions among the bending coordinates to isotope effect are small compared to the diagonal terms such as $f_{i j} \delta g_{j j}$ and $f_{i j}{ }^{2} \delta g_{i j}$ [ cf: Eq. (26), (27), (35), (36), (42), (43)], since $g_{i j} f_{i j} \ll g_{i j} f_{i j}, g_{i j}{ }^{2} \ll f_{i j} f_{i j}$, and $f_{i j}{ }^{2} \ll f_{i j} f_{i j}$. Consequently, the diagonal element approximations [cf: Eq. (34) and (41)] which use only the diagonal terms of F- and G-mattrices are generally within $1 \%$ of the exact values of $\ln \left(\mathrm{s} / \mathrm{s}^{\prime}\right) \mathrm{f}$ at $300 \mathrm{~K}$ for this type of isotopic substitution.

Hydrogen isotope effects are attributable to individual internal valence coordinztes ${ }^{(4)}$, and the isotope effects atributed to individual valence-coordinate force constants are nearly additive at room temperature. The point is illustrated in Table 1. The bond-stretching force constants account for at least $65 \%$ of the total H/D isotope effect at $300 \mathrm{~K}$ and become even more dominant at higher temperature. The relative importance of stretching and bending forces in an end-atom substitution is due to their dominance in the first two term (the terms of $m=1$ and 2 ) in the orthogonal expansion. 


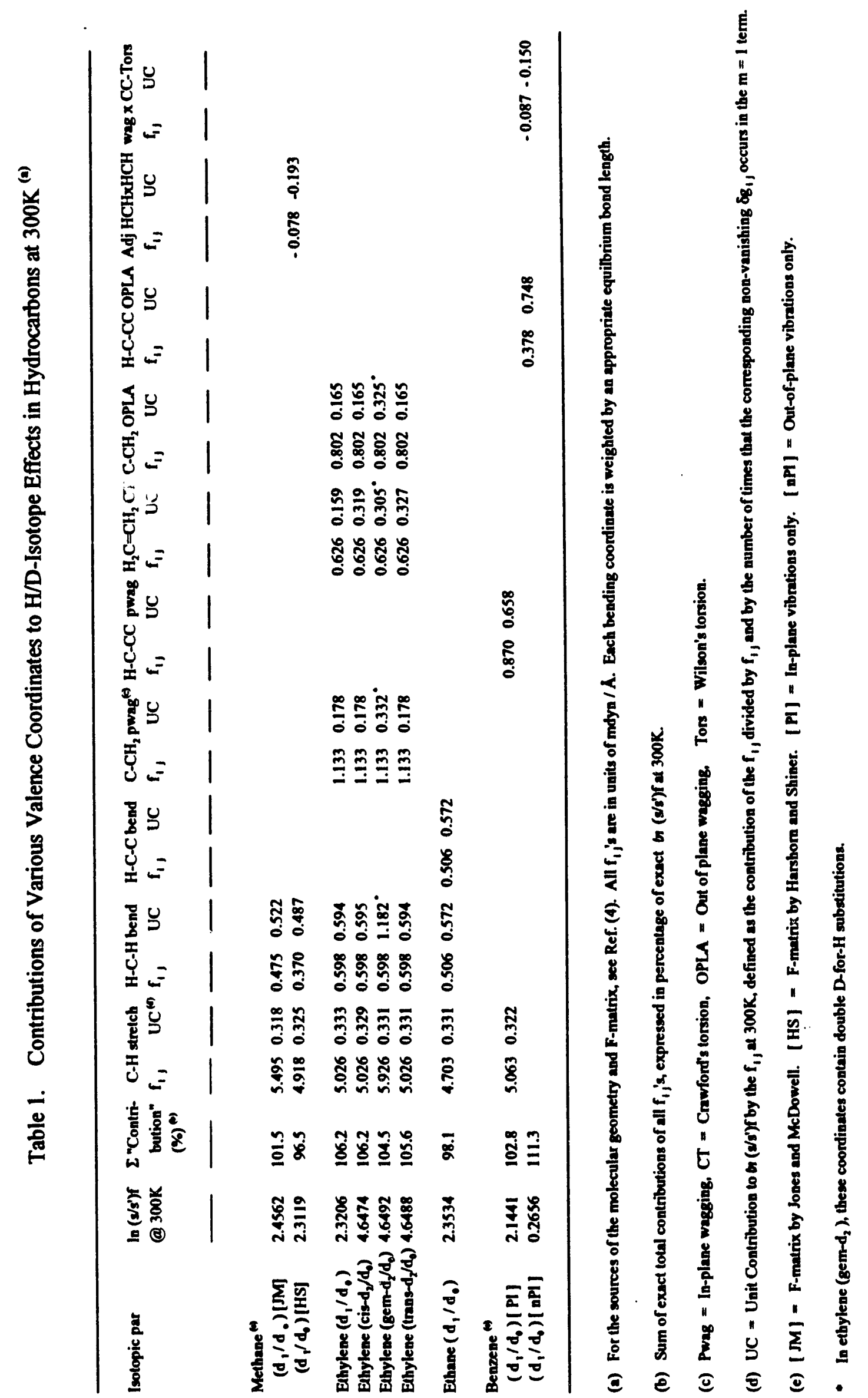


In Table 1 , it is noted that the unit contribution of $\mathrm{C}-\mathrm{H}$ stretching force constant per unit value of (mdyn. $\AA^{-1}$ ) is relatively constant among all molecules, while those for the H-C.H bending coordinate are more scattered. This is a consequence of relatively small effects of the second-order $\left(n_{1}=2\right)$ terms involving the $\mathrm{C}-\mathrm{H}$ coordinate compared to the first-order term. The only significant contribution of $\mathrm{C}-\mathrm{H}$ stretch is $(1 / 24) W_{1}(h c / k T) f_{2} \delta g_{2}$, where $f_{2}$ is the diagonal force constant for the C-H stretching and $\delta g_{1}=$ $1 / \mu_{H}-1 / \mu_{D}=0.496 \mathrm{amu}$.

Generally as far as the H/D isotope effect are concemed, the hydrogenous bond stretching as a valence coordinate is a very good approximation of a normal coordinate. This is because, hydrogen atoms being the lightest atoms, do most of the vibrational motions against the other atoms, and the motions do not kinetically intereact with other valence coordinates except between the adjacent bends that involve the isotopically substituted hydrogen.

Analysis of end-atom isotope effects other than the hydrogen isotope effect is similarly simple and the additivity holds well, although the bond-stretching valence coordinates involving other end-atoms are not as good an approximation of a normal coordinate as the stretching coordinate involving a hydrogen atom.

These observations lead to a simple method for calculating the end-atom isotope effects. ${ }^{(2)}$ It is possible to compute the RPFR at room temperature and above with a reasonable accuracy simply from the atomic masses and the stretching and bending force constants. The method is based on the first-order approximation to the method of Eq. (19):

$$
\ln \frac{s}{s^{\prime}} f \equiv \frac{1}{24}\left(\frac{\hbar c}{k T}\right)^{2} \sum_{\text {sreiches e bends }}^{\sum}\left(W_{i}^{\prime} g_{i i}^{\prime}-W_{i} g_{i i}\right) f_{i i} \text {, }
$$

where $W_{i}^{\prime}$ and $W_{i}$ are the first-order $(m=1)$ modulating coefficients for the lighter and heavier isotopic molecules and found from one of the tables reported in References 105 and 106. Here it is sufficient to use $\lambda_{i}^{\prime} \cong\left(\mu_{m d^{\prime}}+\mu\right) f_{i j}$ and $\lambda_{i} \cong\left(\mu_{-\alpha}+\mu\right) f_{i j}$ as a basis for the look-up values of the range of expansion in the $W$-tables. Here, the assumption, $W_{i}^{\prime} \cong W_{i}$, is adequate.

\section{Deviations from the First Rule of The Mean}

One of the most critical tests of various isotope effect data on a given molecule for the data's internal consistency is the tests on the sum rules for the isotopic vibrational frequencies and those on the rules of the mean for the isotopic thermodynamic data.

The first order sum rule ${ }^{(108)}$ for the frequencies states that, for certain sets of isotopic molecules, a certain algebraic sum of the molecular sums of the squares of harmonic frequencies over the set vanishes. The set of isotopic molecules must satisfy the superposition rule, which requires that the set of molecules can be geometrically superimposed with appropriate signs in such a way that the atoms vanish at all positions. The sum rule is a direct and exact consequence of the assumptions of a harmonic potential, its 
invariance with respect to isotopic substitution, and the equivalence of isotopically substituted positions. The higher order sum rules ${ }^{(105,109)}$ are also based on the same assumptions.

There is a statistical mechanical analogue of each of the sum rules. ${ }^{(103,105,109,110)}$ The most common ones are the rule of the mean related to the first order sum rule and the temperature-independent pre-exponential factor related to the Teller-Redlich product (3n-6) rule. ${ }^{(103)}$ Thus, because of the first order sum rule, the equilbrium constant at room temperature for an isotopic disproportionation reaction such as

$$
\mathrm{H}_{2} \mathrm{O}+\mathrm{D}_{2} \mathrm{O}=2 \mathrm{HDO}
$$

is very close to the high temperature classical limit, $s\left(d_{0}\right) s\left(d_{2}\right) / s\left(d_{1}\right)^{2}=4$. The usually small deviations from the rule of the mean value can be interpreted ${ }^{(6)}$ in the light of the $m=2$ and higher order terms of orthogonal expansion of $\ln \left(\mathrm{s}^{\prime} / \mathrm{s}^{\prime}\right) \mathrm{f}$. It is possible to develop a systematics of the deviations from any $n$-th order rule of the mean and express the deviation in terms of the reciprocal masses of the substituted atoms and the harmonic force constants.

To facilitate a simple identification of the valence coordinates that would make non-vanishing contributions to the $m$-th order deviation from an $n$-th order rule of the mean and of those which do not contribute to the deviation, a new matrix, D-matrix, was introduced. ${ }^{(6)}$ The elements of a D-matrix are zero or unity, depending on whether an interaction between two valence coordinates makes a zero or finite contribution to the deviation. A systematic method for constructing D-matrix was developed. In general, presence of off-diagonal elements in F-matrix among the primarily bending motions, such as bond-angle bending, wagging and bond-torsion, tends to increase the deviation even in the cases of D/H substitutions. In the cases of multiple isotope substitutions in a molecule, proximity of the substituted atomic positions promotes significant couplings of higher order and leads to deviations from the rule of the mean.

\section{II-B-2. Central Atom issitu: Effects}

When an isotope substitution is made at a non-end atom position such as positions 2 and 3 in Figure 1, number of non-vanishing cross-interaction terms in the $m=2$ and higher terms increases exponentially. The $m=2$ term in the orthogonal expansion of $\ln \left(s / s^{\prime}\right) f$ contains the isotopic difference in the trace of $(\mathbf{F G})^{2}$;

$$
\delta \operatorname{Tr}\left(\mathbf{H}^{2}\right)=\operatorname{Tr}\left[\left(\mathbf{F} \mathbf{G}^{\prime}\right)^{2}\right]-\operatorname{Tr}\left[(\mathbf{F G})^{2}\right]
$$

which further consists of the following seven types of terms: (1) $f_{i j}{ }^{2} \delta g_{i j}{ }^{2},(2) f_{i j}{ }^{2} \delta\left(g_{i j} g_{i j}\right),(3) f_{i j} f_{i j} \delta\left(g_{i j} g_{i j}\right)$, (4) $\left(f_{i j} f_{i j}+f_{i j}{ }^{2}\right) \delta\left(g_{i j}{ }^{2}\right)$, (5) $f_{i j} f_{i k} \delta\left(g_{i j} g_{j k}\right)$, (6) $\left(f_{i j} f_{i k}+f_{i j} f_{j k}\right) \delta\left(g_{i j} g_{i k}\right)$, and ( 7$) f_{i j} f_{i j} \delta\left(g_{i j} g_{i t}\right)$. In the end-atom isotope effects, all of these vanish except for those which only involve the diagonal elements 
such as $g_{i j}$ and $g_{j}$ and $g_{i j}$ 's in which $(i, j)$ are adjacent angle-bending coordinates. This is so, even when all off-diagonal $f_{i j}$ 's were non-vanishing. In reality, the $f_{i j}$ elements tend to be negligible between $i$ and $j$ that are few bonds away from each other.

In contrast, in the central atom isotope efects the following $g_{i j}$ 's are in general non-vanishing and isotope-dependent:

$(i, j)=$ two stretches sharing the central atom,

$(i, j)=a$ stretch and $a$ bend sharing a bond involving the central atom,

$(i, j)=a$ stretch and a bend sharing the central atom but not a bond,

$(i, j)=$ two bends sharing the central atom,

and $(i, j)=$ two bends sharing a bond involving the central atom.

This has a consequence of extending the effects of an isotope substitution at a non-terminal atomic position down several bonds instead of merely to one or two bonds. The question of down how many chemical bonds an isotope substitution at an atomic position carries its effects depends on an interplay between the off-diagonal $g_{i j}$ and interaction force constants $f_{\mathrm{b}}$. We examined these effects using prototype molecules, $\mathrm{CO}_{2}, \mathrm{H}_{2} \mathrm{O}, \mathrm{CH}_{4}, \mathrm{C}_{2} \mathrm{H}_{4}, \mathrm{C}_{2} \mathrm{H}_{6}$ and $\mathrm{C}_{6} \mathrm{H}_{6}{ }^{(3.12 .13)}$ The stretching forces dominate the isotope chemistry of carbon and oxygen, accounting for $70 \%$ to $90 \%$ of the RPFR among them. In general, valence-bond contributions for the central atom isotope effects are far less additive than those of the end-atom isotope effect. Contributions of individual force constants to the isotope effect do not obey the rules of the means as well as those for the end-atom isotope effects. The method of D-matrix is especially useful for finding the molecular features that cause the deviations from the rules of the means.

\section{Isotope Effects at the Center of Highly Symmetric Molecules}

Although the correlation of isotope effects with various features of a molecule is generally not a straightforward task, the analysis of effects of an isotopic substitution at an atomic position located on all symmetry elements of a highly symmetric molecule leads to a clear-cut correlation due, in large part, to an extensive factorization of the vibrational secular equation. ${ }^{(34)}$ The structure of block-factoring of the secular equation is isotope-independent for these substitutions. This leads to a simple formula for the isotope shift in every vibrational frequency, in which all terms are expressed as an explicit function of the elements of F- and G-matrices.

In the highly symmetric molecules such as those in point groups $T_{d}, O_{k}$, and $D_{s h}$, many chemical bonds around the central atom are at $90^{\circ}$ from each other, and many planes contained in the molecule are perpendicular with each other. This leads to many vanishing $g_{i j}{ }^{\prime} s$. A central atom substitution such as one at $\mathrm{X}$ in $\mathrm{XY}, X Y_{6}$, and $X Y_{2} Z_{5}$ does not change the molecular symmetry. Thus,, for the $X^{\prime} / X$-isotope effect in $X_{2} Y_{3}$, the irreducible representation,

$$
\Gamma=2 A_{1}^{\prime}+2 A_{2}^{\prime}+3 E_{1}^{\prime}+2 E_{2}^{\prime}+E_{1}^{\prime}+E_{2}^{\cdot} \text {, }
$$


is unchanged by the $X^{\prime} / X$-substitution. Furthermore, only the trwo-dimensional block of $A_{2}^{\prime}$, the three-dimensional block of $E_{1}^{\prime}$ and the two-dimensional block of $E_{2}^{\prime}$ are isotope-dependent, and every element of $G$-matrix in the $A_{2} ;, E_{1}^{\prime}$ and $E_{2}^{\prime}$ blocks contains a term of $\mu_{x}$, the reciprocal mass of atom $X$. Thus, each element of the corresponding $\mathbf{H}$-matrix is a uniformly linear function of $\mu_{x}$;

$$
H_{i j}=a_{i j} \mu_{x}+b_{i j}
$$

Since all algebraic equations of the orders up to three are analytically solvable, we have a means for obtaining the expressions for all vibrational eigenvalues of both isotopic molecules and their isotope shifts. For example, take a 2-dimensional block. The vibrational secular equation is of the form,

$$
\lambda^{2}-\alpha \lambda+\beta=0 \text {, }
$$

where $\alpha$ and $\beta$ are

and

$$
\begin{aligned}
& \alpha=G_{11} F_{11}+G_{22} F_{22}+2 G_{12} F_{12}, \\
& \beta=\left(G_{11} F_{11}-G_{12}{ }^{2}\right)\left(F_{11} F_{22}-F_{12}{ }^{2}\right)
\end{aligned}
$$

They are in the forms,

and

$$
\begin{aligned}
& \alpha=b_{1} \mu_{x}+c_{1}, \\
& \beta=a_{0} \mu_{x}^{2}+b_{0} \mu_{x}+c_{0},
\end{aligned}
$$

in which the coefficient $a_{0}$ is a function of the force constants only, while $b_{i}$ and $c_{i}$ are functions of force constants and also linear functions of $\mu_{\gamma}$ and $\mu_{z}$.

Differentiating Eq. (54) and solving for $\delta \lambda$, one obtains

$$
\delta \lambda=\frac{\lambda \delta \alpha-\delta \beta}{2 \lambda-\alpha}
$$

where $\delta \lambda=\lambda^{\prime}-\lambda, \delta \alpha=\alpha^{\prime}-\alpha$, and $\delta \beta=\beta^{\prime}-\beta$. Thus,

$$
\begin{aligned}
& \delta \alpha=b_{1} \delta \mu_{x} \\
& \delta \beta \cong\left(2 a_{0} \mu_{x}+b_{0}\right) \delta \mu_{x}
\end{aligned}
$$

and

$$
\left(\frac{\hbar c}{k T}\right)^{2} \delta \lambda=\delta u^{2} \equiv 2 u \delta u
$$

Equations (58b) and (59) hold well when $X$ is a heavy atom. We see that $\delta \mu_{X}$ factors out of the numerator on the right-hand side of Eq. (57). The $\lambda$ 's in Eq. (57) can expressed in terms of the same geometric and force constant parameters as those used for the expressions of $\alpha$ and $\beta$. We thus have a means for explicitly correlating $\lambda$ and $\delta \lambda$, and consequently $u$ and $\delta u$, with the parameters such as molecular geometry, isotopic masses and the force constants.

Similarly, for a 3-dimensional blocks of vibrational secular equation, $\lambda$ and $\delta \lambda$ can be expressed in terms of the molecular parameters. ${ }^{(34)}$ 
Then, the method of using the symmetry coordinates for the highly symmetric molecules can be combined with Bigeleisen's $G(u)-S(u)$ approximation ${ }^{(111,103)}$ for $\ln \left(s / s^{\prime}\right) f$;

$$
\ln \left(\frac{s}{s^{\prime}}\right) r=\sum_{i} G\left(u_{i}\right) \delta u_{i}\left[1+\frac{S\left(u_{i}\right)}{2 G\left(u_{i}\right)} \frac{\delta u_{i}}{u_{i}}+\cdots\right]
$$

where

$$
G(u)=\frac{1}{2}-\frac{1}{u}+\frac{1}{e^{u}-1}
$$

and

$$
S(u)=\frac{1}{u}-\frac{u e^{u}}{\left(e^{u}-1\right)^{2}}
$$

If $u_{i}$ and $\delta u_{1}$ were calculable, even the first term of the $G(u) \delta u$-approximation would generally give an order of magnitude better precision than any other correlation method. However, the precision required for a satisfactory evaluation of $\delta u$ is far more stringent than the requirement for $u$. The present method for the explicit relationships for $\lambda_{1}$ and $\delta \lambda_{1}$ presented in this section provides a reliable means for isotope effect correlation for the substitutions at the highly symmetric center of molecules having a high symmetry. 


\section{Correlation of Zero-Point Energy with Molecular Froces and Structures}

We studied various theoretical aspects of vibrational zero-point energy (ZPE), its isotope shift, $\delta(Z P E)$, and phase-shifts of the isotope shift, $\Delta \delta(Z P E){ }^{(13,19,22,23,25.27,20.36)}$

The ZPE is defined as

$$
Z P E=\frac{1}{2} h \sum_{i}^{f} v_{i}=\frac{\pi}{2} \sum_{i}^{f} \lambda_{i}^{12},
$$

where $f$ is vibrational degree of freedom, and $\lambda_{i}$ is the $i$-th vibrational eigenvalue obtained by solving

$$
|\mathbf{H}-\lambda I|=|\mathbf{G ~ F}-\lambda I|=0
$$

Since it is difficult to accurately approximate the function $f(x)=x$ in terms of powers of $x^{1 / 2}$, there had not been an accurate approximation for the ZPE in terms of the elements of $G$ and $F$ matrices. We developed such an approximation method based on the orthogonal polynomial series.

\section{II-A. The ZPE as Explicit Function of Molecular Structure and Molecular Forces}

In this part, we will first present the development of the mathematical tool and then give a summary of results of various applications to various isotope effect and non-isotope effect problems.

\section{III-A-1. Development of Mathematical Tools}

We based our development ${ }^{(19)}$ of mathematical theory for this purpose on Lanczos' orthogonal polynomial expansion ${ }^{(104)}$ of function $x^{1 / 2}$ as follows:

$$
x^{12}=\sum_{m=0}^{n} \alpha_{m} x^{m} \quad[0 \leq x \leq 1]
$$

where

$$
\alpha_{m}=\frac{C_{n}^{m}}{2 m-1}\left[\sum_{j=0}^{n} \frac{C_{n}^{j}}{2 j-1}\right]^{-1},
$$


in which $\mathrm{C}_{\mathrm{a}}^{\mathrm{m}}$ is the coefficient of the $\mathrm{m}$-th order term in a Jacobi polynomial of order $\mathrm{n}$ defined in the range, $0 \leq x \leq 1$.

Let the normal frequency spectrum of an $\mathrm{N}$-atomic molecule be represented by the following ascending sequence of vibrational eigenvalues;

$$
\lambda_{1}, \lambda_{2}, \ldots, \lambda_{i}, \ldots, \lambda_{1}\left(-\lambda_{\max }\right)
$$

in which degenerate eigenvalues are counted separately. Corresponding to the sequence (67), we create a normalized sequence, that is, normalized to a certain value $\lambda_{0}$, which would be determined later:

$$
x_{1}, x_{2}, \ldots, x_{i}, \ldots, x_{1}\left(-x_{\max }\right)
$$

where

$$
x_{i}=\lambda_{i} / \lambda_{0}
$$

and

$$
x_{\operatorname{mex}}=x_{1}=\lambda_{\max } / \lambda_{0}
$$

The parameter $\lambda_{0}$ is completely arbitrary at this point. In order to take advantage of Eq. (65), however, we define the range of expansion, $\xi$, such that

$$
x_{\max } \leq \xi \text {. }
$$

Then, the ZPE can be approximated by

$$
Z P E=\frac{h}{2} \sum_{i}^{f} \lambda_{i}^{1 / 2} \cong \frac{h}{2} \lambda_{0} \sum_{m=0}^{n} \frac{\alpha_{m}}{\lambda_{0}^{m}} \sum_{i}^{f} \lambda_{i}^{m}
$$

where $\alpha_{\mathrm{m}}$ is

$$
\alpha_{m}=\frac{1}{2 m-1} \frac{C_{n}^{m}}{\xi_{m}}\left[\sum_{m=0}^{n} \frac{1}{2 j-1} \frac{C_{m}}{\xi^{j}}\right]^{-1}
$$

Further, we have

$$
\left.\sum_{i}^{f} \lambda_{i}^{m}=\operatorname{Tr}\left(\mathbf{H}^{m}\right)=\operatorname{Tr}[\mathbf{F G})^{m}\right]
$$

For $n=2$, Eq. (72) becomes

$$
Z P E=\frac{h}{2} \sum_{i=1}^{f}\left[b_{0}\left(\lambda_{0}, \xi\right)+b_{1}\left(\lambda_{0}, \xi\right) \lambda_{i}+b_{2}\left(\lambda_{0}, \xi\right) \lambda_{i}^{2}\right]
$$

where

$$
\begin{aligned}
& b_{0}=a_{0} \lambda_{0}^{1 / 2} \xi^{2} / D \\
& b_{1}=a_{1} \lambda_{0} \cdot 1 / 2 \xi / D \\
& b_{2}=a_{2} \lambda_{0}^{-1 / 2} / D
\end{aligned}
$$

in which

$$
D=a_{0} \xi^{2}+a_{1} \xi+a_{2}
$$

The best values of the parameters $\lambda_{0}$ and $\xi$ would be those that minimize the error defined by

$$
S=\Sigma_{i} w\left(\lambda_{1}\right) \Delta^{2}
$$

where $\omega\left(\lambda_{1}\right)$ is a weighting function, which we took to be proportional to $\lambda_{1}^{k}$,

$$
w\left(\lambda_{1}\right)=A \lambda_{1}^{*},
$$


and

$$
\Delta_{i}=b_{0}\left(\lambda_{0}, \xi\right)+b_{1}\left(\lambda_{0}, \xi\right) \lambda+b_{2}\left(\lambda_{0}, \xi\right) \lambda^{2}-\lambda_{i}^{1 / 2}
$$

For a given $k$, an optimum set of $\left(\lambda_{0}, \xi\right)$ is obtained by simultaneously solving

$$
\text { and } \quad \begin{aligned}
& \partial S / \partial \lambda_{0}=0 \\
& \partial S / \partial \lambda_{0}=0
\end{aligned}
$$

Three cases were explored: Case A, for which a constraint $\lambda_{0}=\lambda_{\max }$ was set; case $B$, another special case in which the range of expansion $\xi$ H $_{a j}$ equated to $x_{\text {and }} ;$ and case $C$ in which $\xi$ and $\eta\left(=\lambda_{\max } / \lambda_{0}\right)$ were treated as independent of each other.

The optimization of $S$ yields the following sets of solutions. Case A: three positive roots for $\xi$ for every $k$ value tested, ranging from $k=0$ to $10^{6}$. Each of three roots, $\xi(a 1), \xi(22)$, and $\xi(23)$, leads to a distinct approximation. Case B: three $k$-dependent positive roots for $\xi$ (and the corrsponding values of $\lambda_{0}$ ), which are designated as $\xi(\mathrm{bl}), \xi(\mathrm{b} 2)$ and $\xi(\mathrm{b} 3)$, and two $k$-independent roots, $\xi(b 4)$ and $\xi(05)$. The three roots, $\xi(\mathrm{b} 1), \xi(\mathrm{b} 2)$, and $\xi(\mathrm{b} 3)$, lead to an identical approximation, while $\xi(\mathrm{b} 4)$ and $\xi(\mathrm{b} 5)$ give two distinct approximations. Case $C$ : two positive roots, $[\xi(c 1)$ and $\eta(c 2)]$ and $[\xi(c 2), \eta(c 2)]$. The two roots produce an identical approximation.

Some representative values of the solutions of Eqs. $(83 \mathrm{a}, \mathrm{b})$ for each of the three cases are shown in

\begin{tabular}{|c|c|c|c|}
\hline$k$ & $t(a 1)$ & $t(22)$ & $5(23)$ \\
\hline $\begin{array}{r}0.0 \\
0.5 \\
1.0 \\
1.5 \\
2.0 \\
2.5 \\
3.0 \\
3.5 \\
4.0 \\
4.5 \\
5.0 \\
10.0 \\
15.0 \\
20.0 \\
.0\end{array}$ & $\begin{array}{l}0.84912 \\
0.87648 \\
0.89883 \\
0.91803 \\
0.93477 \\
0.94949 \\
0.96253 \\
0.97415 \\
0.98457 \\
0.99396 \\
1.00245 \\
1.05729 \\
1.08531 \\
1.10231 \\
1.17157\end{array}$ & $\begin{array}{l}1.83437 \\
1.66532 \\
1.70655 \\
1.75990 \\
1.81041 \\
1.85561 \\
1.89549 \\
1.93065 \\
1.96172 \\
1.98931 \\
2.01394 \\
2.16422 \\
2.23518 \\
2.27635 \\
2.43050\end{array}$ & $\begin{array}{l}2.14721 \\
3.17950 \\
3.75241 \\
4.16045 \\
4.47011 \\
4.71430 \\
4.91221 \\
5.07603 \\
5.21394 \\
5.33167 \\
5.43338 \\
5.99785 \\
6.23723 \\
6.36954 \\
6.82843\end{array}$ \\
\hline
\end{tabular}
Tables 2, 3, and 4.

Table 2. Best Values of $\xi$ for Case $A_{1} \lambda_{0}=\lambda_{\ldots}{ }^{(10)}$

\footnotetext{
- The value of $k$ higher than $10^{\circ}$, beyond which the
} roots are within $10^{-3}$ of their asymptotic limits.
Table 3. Best Values of $\xi$ for Case B, $\xi=\lambda_{\alpha} / \lambda_{0}{ }^{(19)}$

\begin{tabular}{cccc}
\hline$k$ & $k$ (b1) & $k$ (b2) & $\xi(\mathrm{b} 3)$ \\
\hline 0.0 & 0.86717 & 1.74608 & 19.83636 \\
0.5 & 0.89793 & & \\
1.0 & 0.92622 & 1.56635 & 20.75194 \\
1.5 & 0.95022 & & \\
2.0 & 0.97020 & 1.46361 & 21.22786 \\
2.5 & 0.98672 & & \\
3.0 & 1.00030 & 1.40427 & 21.47268 \\
3.5 & 1.01140 & & \\
4.0 & 1.02039 & 1.36867 & 21.60478 \\
4.5 & 1.02763 & & \\
5.0 & 1.03338 & 1.34715 & 21.67812 \\
6.0 & 1.04140 & & \\
7.0 & 1.04602 & & \\
8.0 & 1.04836 & & \\
9.0 & 1.04921 & &
\end{tabular}


Table 4. Best Values of $\xi$ and $\eta$ for Case $C .{ }^{(19)}$

\begin{tabular}{ccccc}
\hline$k$ & $\xi(c 1)^{a}$ & $\eta(c 1)^{a}$ & $k(c 2)$ & $n(c 2)$ \\
\hline 0.172663 & 2.43838 & 1.44347 & & \\
0.5 & 3.25879 & 1.31960 & & \\
1.0 & 3.85621 & 1.24617 & 11.56689 & 3.73796 \\
1.5 & 4.26491 & 1.20228 & & \\
2.0 & 4.57027 & 1.17215 & 9.98292 & 2.56034 \\
2.5 & 4.80892 & 1.14998 & & \\
3.0 & 5.00120 & 1.13293 & 9.20211 & 2.08457 \\
3.5 & 5.15966 & 1.11939 & & \\
4.0 & 5.29264 & 1.10836 & 8.73296 & 1.82881 \\
5.0 & 5.40588 & 1.09920 & & \\
7.0 & 5.78869 & 1.06974 & & \\
10.0 & 6.04237 & 1.05142 & & \\
15.0 & 6.26962 & 1.03575 & & \\
20.0 & 6.39496 & 1.02740 & & \\
0 & 6.82843 & 1.00000 & &
\end{tabular}

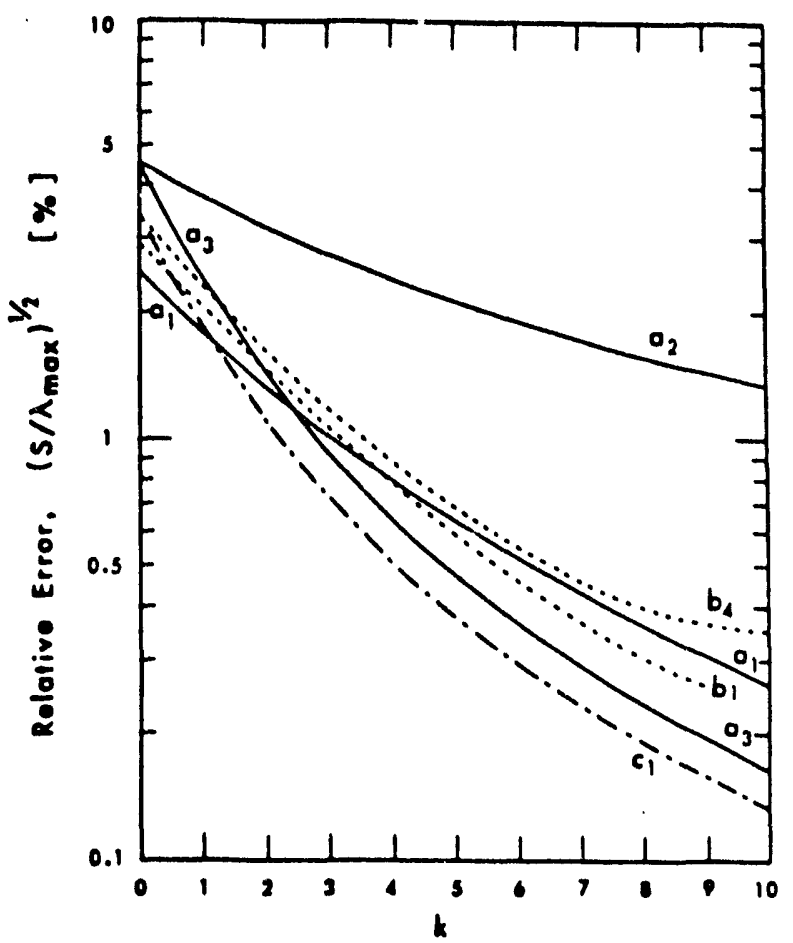

Figure 3. Relative errors in the ZPE approximations for the model syatem using various roots of Eqs. $(832, b)^{(1)}$

For the model system which has its frequency distribution according to Eq. (82), the relative error, $\sigma$, of an approximation can be written as

$$
\frac{\sigma_{Z P E}}{Z P E}=\frac{2 k+3}{2 k+2}\left(\frac{S}{\lambda_{\max }}\right)^{1 / 2}
$$

This function is plotted in Figure 3. 


\section{III-A-2. Zero-Point Energy Approximation for n-Alkanes}

For real molecules, actual precision of approximation depends on the particular distribution of frequencies. The best second-order approximation based on the Chebyshev polynomial $T_{n}{ }^{*}(x)$ for $n$-para-

Table 5. Beat Approximations for a-Paraffins ${ }^{\infty}$

\begin{tabular}{clcc}
\hline \multicolumn{2}{c}{ approx } & complete spectra & out-of-plane epectra \\
\hline A & parameters & al $(k=0)$ & $-3(k=0)$ \\
& precision & $-3.1 \%\left(C_{1}\right)$ to & $-1.3 \%\left(C_{2}\right)$ to \\
& & $-0.3 \%\left(C_{10}\right)$ & $-0.5 \%\left(C_{14}\right)$ \\
B & parameters & $b 1(k=0)$ & $b 1(k=0)$ \\
& precision & $-2.5 \%\left(C_{1}\right)$ to & $-3.1 \%\left(C_{1}\right)$ to \\
& & $-0.03 \%\left(C_{1}\right)$ & $-2.7 \%\left(C_{14}\right)$ \\
C & parameters & $c l(k=0.173)$ & $c 1(k=0.173)$ \\
& precision & $+0.1 \%\left(C_{1}\right)$ to & $+0.6 \%\left(C_{3}\right)$ to \\
& & $+5.8 \%\left(C_{10}\right)$ & $1.4 \%\left(C_{14}\right)$
\end{tabular}
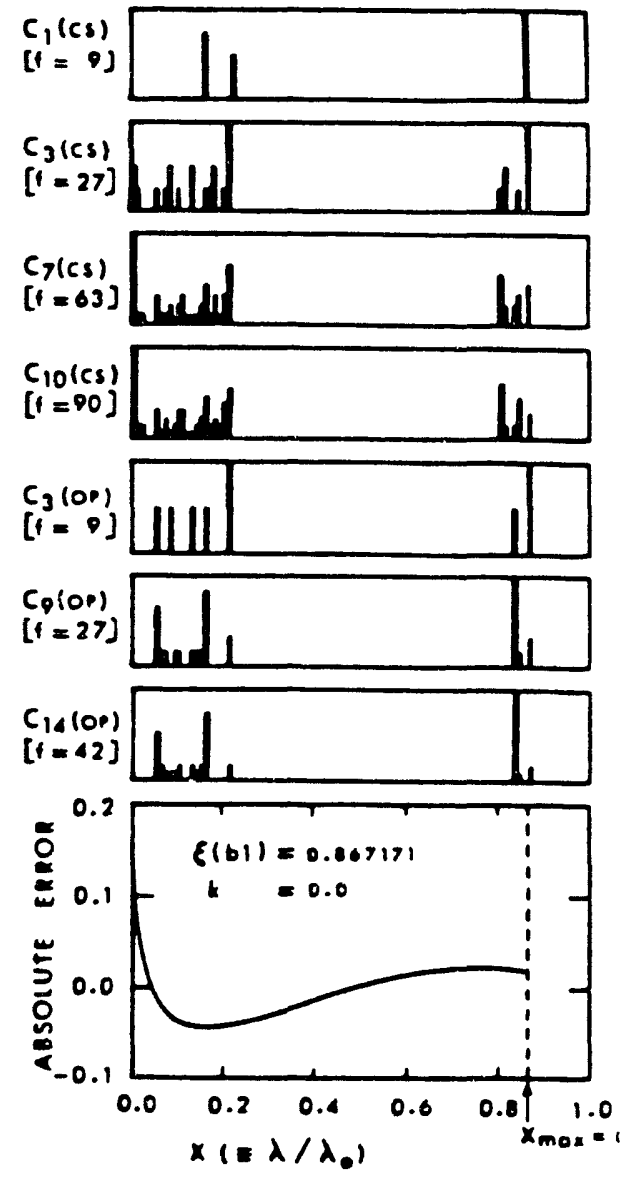

Figure 4. Abeolute arror and distribution of $\lambda_{i}$ as functions of $x$ for Case $B$ at $k=0$. (2)

ffins up to $\mathrm{C}_{14} \mathrm{H}_{30}$ are summarized in Table 5 . Among all cases we studied (22) the best results for n-parrafins were obtained by equating the range of expansion to the largest normalized eigenvalue (CaseB) and setting $k=0$; the absolute error is $2.5 \%$ for $\mathrm{C}_{1}$ and lower than $1 \%$ for the molecules larger than propane. These results which are entirely different from the one expected from Figure 3 are due to the difference in the frequency distribution. Figure 4 illustrates this for Case $B$ at $k=0$. The unit interval of the histogram is $\Delta x=0.01$, and the heights are normalized to wity for the most populuous interval. 


\section{III-A-3. Hydrogen Isotope Effects in ZPE and Linear Frequency Sum Rules}

For an isotope shift in ZPE, let us choose to use the same set of approximation parameters for the ZPE's of both isotopic molecules. Then, Eq. (75) becomes

$$
\begin{array}{ll}
\delta(\mathrm{ZPE}) \cong \frac{\hbar}{2}\left[b_{1} \delta\left(\Sigma \lambda_{i}\right)+b_{2} \delta\left(\Sigma \lambda_{i}{ }^{3}\right)\right] \\
\text { where } \quad & \delta\left(\Sigma \lambda_{1}\right)=\operatorname{Tr}\left(H^{\prime}\right)-\operatorname{Tr}(H), \\
& \delta\left(\Sigma \lambda_{1}\right)=\operatorname{Tr}\left(H^{\prime 2}\right)-\operatorname{Tr}\left(H^{2}\right)
\end{array}
$$

and

$$
\mathbf{H}^{\prime}=\mathbf{G}^{\prime} \mathbf{F} \text { and } \mathbf{H}=\mathbf{G} \mathbf{F} \text {. }
$$

The best approximation parameters for all single and multiple $\mathrm{D}$-for- $\mathrm{H}$ substitutions in $\mathrm{H}_{2} \mathrm{O}, \mathrm{HCHO}, \mathrm{CH}_{4}$, $\mathrm{C}_{2} \mathrm{H}_{4}, \mathrm{C}_{2} \mathrm{H}_{6}$, and $\mathrm{C}_{6} \mathrm{H}_{6}$ were found among the Cases of $C .{ }^{(23)}$ In Figure 5, the ordinate is

$$
\frac{\Delta}{\lambda_{\max }^{1 / 2}}=\frac{\frac{1}{2} h v_{\text {approx }}-\frac{1}{2} h v}{\frac{1}{2} h v_{\max }}
$$

The actual errors for $\delta(Z P E)$ of real molecules are strongly dependent on the distribution of eigenvalue shifts.

Generally, the approximations for $\delta(Z P E)$ of all molecules can be analyzed by means of classification of the valence coordinates into four categories;

$n_{1}=$ number of $\mathrm{H}-\mathrm{X}$ bonds,

$n_{b}=$ number of independent $H-X-Y$ angles,

$n_{1}=$ number of torsional and other out-ofplane coordinates involving hydrogen,

$n_{r}=$ number of coordinates which do not directly involve motions of hydrogens.

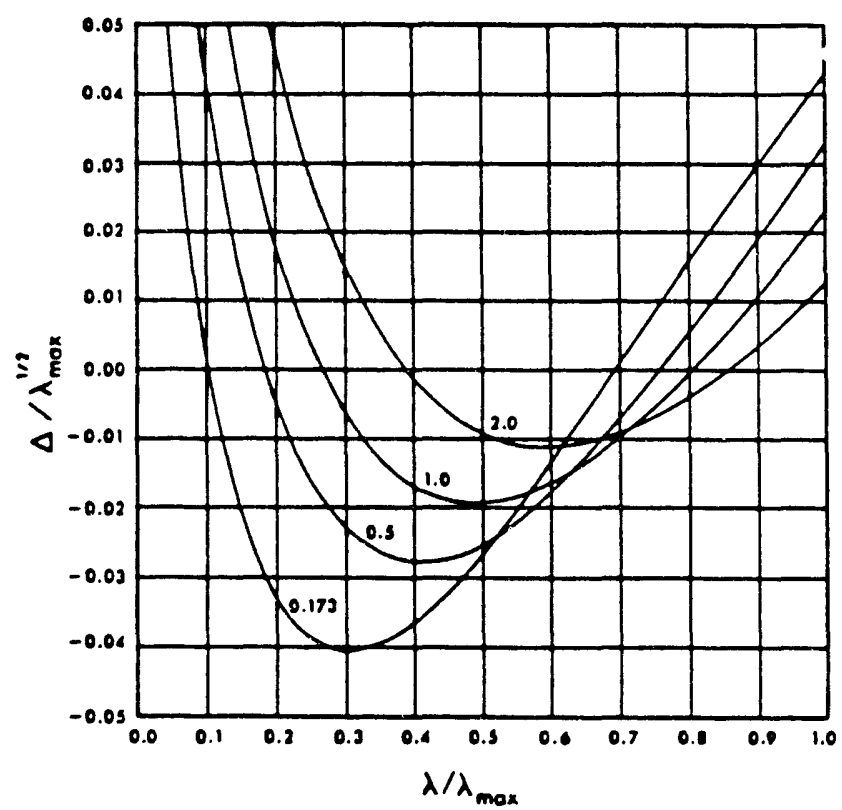

Figure 5. Normalized error $\Delta \lambda z^{12}$ as a function of normalized eigenvalue $\lambda / \lambda \ldots$ for approximations of Type $C$. Numbers on the curves are $k$ values. ${ }^{\text {(23) }}$ 
Thus, at $k=0.173$ for Case $C$, the least-squares fit of the best-k data on the prototype molecules we studied yields ${ }^{(2)}$

$$
k_{m}=0.019 n_{1}-0.082\left(n_{b}+n_{t}\right)+0.994
$$

Linear frequency sum rule was derived on the basis of these orthogonal expansion. ${ }^{(23)}$ The rule approximately holds for any set of isotopic molecules that satisfies a second-order frequency sum rule. The first-order ${ }^{(112)}$ and second-order ${ }^{(109)}$ frequency sum rules exactly holds under the Bom-Oppenheimer approximation and, respectively, correlate the sums of the squares and the fourth powers of vibrational frequencies of isotopic molecules. From Eq. (85), we have

$$
\delta \Sigma v_{i} \equiv \frac{1}{2 \pi}\left[b_{1} \delta \Sigma \lambda_{i}+b_{2} \delta \Sigma \lambda_{i}{ }^{2}\right]
$$

The second-order sum rule may be generally written as

$$
\Sigma_{j} \alpha_{j}\left(\delta \Sigma_{j} \lambda_{i}{ }^{2}\right)_{j}=0 \text {, }
$$

where $\alpha_{j}$ is a sum-rule coefficient for the $j$-th isotopic pair, and the sum over $j$ is taken over all pairs involved in the sum rule. When a second-order rule holds, then a first-order rule also holds among the same set of isotopic molecules and with the same coefficient. Thus, if Eq. (91) holds, ther we also have

$$
\Sigma_{j} \alpha_{j}\left(\delta \Sigma \lambda_{j}\right)_{j}=0 \text {. }
$$

If we define a sum rule difference, $\Delta_{\boldsymbol{\beta}}$, by

$$
\Delta_{\delta}=\Sigma_{j} \alpha_{j}\left(\delta \Sigma \lambda_{i}\right)_{j},
$$

Eq. (90) then leads to

$$
\Delta_{\text {s. mox }}=\frac{1}{2 \pi}\left[b_{1} \Sigma_{j} \alpha_{j}\left(\delta \Sigma \lambda_{1}\right)+b_{2} \Sigma_{j} \alpha_{j}\left(\delta \Sigma \lambda_{i}^{2}\right)\right]
$$

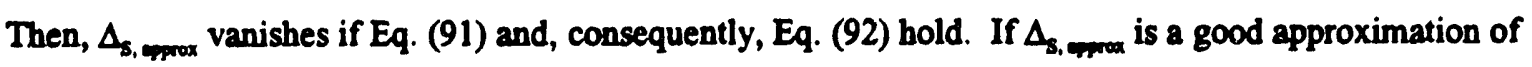
$\Delta_{\mathbf{s}}$, the latter should vanish approximately. Thus

$$
\Sigma_{j} \alpha_{j}\left(\delta \Sigma v_{i}\right)_{j} \equiv 0
$$

We called this relation an approximate linear frequency sum rule.

The error made in equating $\sum \alpha_{j}\left(\delta \Sigma v_{i}\right)$ to zero, $\varepsilon\left(\Delta_{s}\right)$, is

$$
\varepsilon\left(\Delta_{\delta}\right)=\Delta_{\delta}-\Delta_{\text {s.w. }}-\Sigma_{j} \alpha_{j} \varepsilon\left[\left(\delta \Sigma v_{i}\right)_{j}\right]
$$

where $\varepsilon\left[\left(\delta \Sigma v_{i}\right)_{j}\right]$ is the error in $\left(\delta \Sigma v_{i}\right)$. Since the relative errror for $\delta \Sigma v_{i}$ varies approximately linearly with the extent of deuteration, the absolute error may be written as

$$
\varepsilon\left[\left(\delta \Sigma v_{i}\right)_{j}\right] \cong(\beta j+\gamma)\left(\delta \Sigma v_{i}\right)_{j, \ldots},
$$

where $j$ may be taken as $j$ in $\left(d_{j+1} / d_{j}\right)$ or in $\left(d_{j} / d_{0}\right)$. Any sum rule expression can be rewritten by using only the pairs of the form $\left(d_{+1} / d_{)}\right)$or only the pairs of the form $\left(d_{j} / d_{0}\right)$. Therefore, no generality is lost by the approximate linear rule, Eq. (95), by attaching the special meaning to $j$ as introduced by Eq. (97). Substitution of Eq. (97) into Eq. (96) and the use of Eqs. (90) - (94) yield

$$
\varepsilon\left(\Delta_{s}\right) \equiv \beta \Sigma_{j} \alpha_{j}\left(\delta \Sigma v_{i}\right)_{j}
$$


$\left(\delta \Sigma v_{i}\right)_{j}$ 's for monosubstitutions ( $\left.d_{j+1} / d_{j}\right)$ of equivalent atoms are alike, $\Sigma j \alpha_{j}$ is zero for any set satisfying the first-order sum rule, and the factor $\beta$ is on the order of $10^{-2}$. Therefore, $\varepsilon\left(\Delta_{s}\right)$ is likely to be a generally small quantity. Some examples follow:

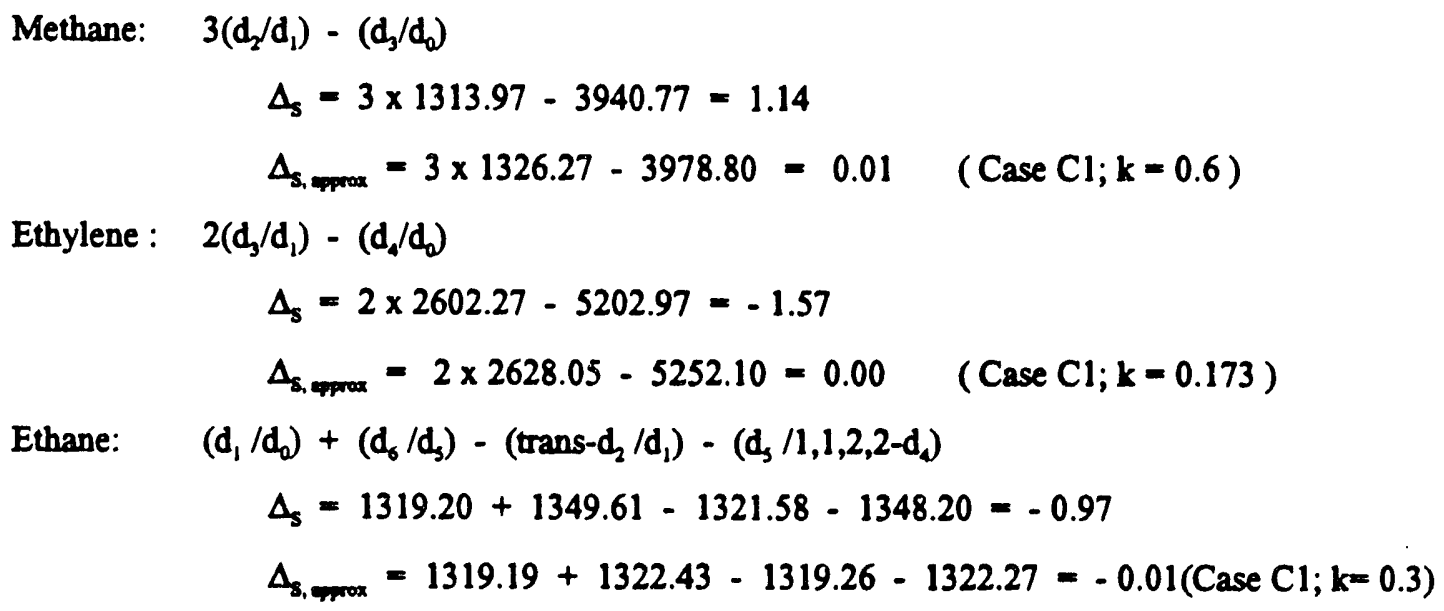

The "law of the mean" for the isotopic ZPEs ${ }^{(113)}$ incorrectly predicts that the sums of isotopic frequencies vanish (unconditionally) for all combinations of the isotopic pairs for which the number of deuteriums cancel. The rules presented here are applicable only for the sets on which a second-order rule holds and yield a precision which is below $0.1 \%$.

\section{III-A-4. Additivity for Zero-Point Energy}

The concept of additivity for the vibrational ZPE is an old one. (114-122) However, the earlier attempts were empirical in nature and without much theoretical foundation. Most of them used a least-squares procedure on the experimentally observed fundamentals and thus lack internal consistency and a common basis for comparison between different authors.

The present orthogonal approximation for the ZPE has provided the needed theoretical foundation. ${ }^{(25)}$ We showed that the earlier rules with varying levels of sophistication can be derived as special cases of the more general rule. From Eq. (75), we obtain

$$
\begin{aligned}
& \frac{2}{\hbar}(Z P E) \cong \sum_{i} C_{i}^{0}+b_{1} \sum_{i<j} \sum_{j} C_{i j}^{1}+b_{2} \sum_{i<} \sum_{j} \sum_{m=1}^{4} C_{i j}^{2}(m)
\end{aligned}
$$

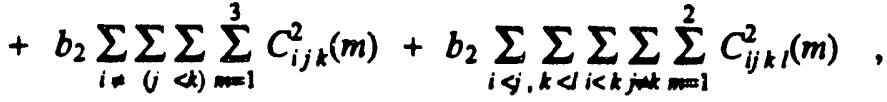

where

$$
\begin{aligned}
& C_{i}{ }^{0}=\left(g_{i i} f_{i i}\right)^{1 / 2}, \\
& C_{i j}{ }^{1}=2 g_{i j} f_{i j}, \\
& C_{i j}{ }^{2}(1)=2 g_{i i} g_{j j} f_{i j}{ }^{2}, \\
& C_{i j}{ }^{2}(2)=4 g_{i j} f_{i j}\left(g_{i j} f_{i i}+g_{i j} f_{j j}\right),
\end{aligned}
$$




$$
\begin{aligned}
& C_{i j}{ }^{2}(3)=2 g_{i j}{ }^{2} f_{i j}{ }^{2}, \\
& C_{i j}{ }^{2}(4)=2 g^{2}{ }^{2} f_{i i} f_{j j}, \\
& C_{i j k}{ }^{2}(1)=4 g_{i j} g_{j k} f_{i j} f_{i k}, \\
& C_{i j k}{ }^{2}(2)=4 g_{i j} g_{i k} f_{i i} f_{j k}, \\
& C_{i j k}{ }^{2}(3)=4 g_{i j} g_{i k} f_{i j} f_{i k}, \\
& C_{i j k 1}{ }^{2}(1)=4 g_{i j} g_{k 1} f_{i 1} f_{j k}, \\
& C_{i j k 1}{ }^{2}(2)=4 g_{i j} g_{k 1} f_{i k} f_{j 1},
\end{aligned}
$$

Each of these C-terms has a physical meaning and can be logically prorated and assigned to the valence coordinates involved in them. For instance, one half of the term, $C_{i j}{ }^{1}=2 g_{i j} f_{i j}$ can be assigned to each of the coordinates $i$ and $j$. A generalization of such proration for all first-order and second-order C-terms contained in Eq. (99) leads to an expression,

$$
Z P E \cong \frac{\hbar}{2}\left[\Sigma_{p} N_{p} \Gamma_{p}+\Sigma_{(\infty)} N_{(m)} \Gamma_{(m)}\right] \quad,
$$

where $\mathrm{N}_{\mathrm{p}}$ is the number of valence coordinates of type $\mathrm{p}$ (e.g., C-H stretch, $\mathrm{H}-\mathrm{C}-\mathrm{H}$ bend in $\mathrm{CH}_{2}$ group) contained in the molecule, and $\Gamma_{p}$ is the diagonal (i.e., non-interactive) contribution of one of the

\begin{tabular}{|c|c|c|c|c|c|c|}
\hline \multicolumn{2}{|r|}{ types of coordinates and interections } & \multirow[b]{2}{*}{$N_{p}$ and $N_{(y)}$} & \multicolumn{4}{|c|}{ unit contributions and corrections, $\mathrm{cm}^{-1}$} \\
\hline $\begin{array}{c}\text { SS.1 } \\
\text { enction }\end{array}$ & deacription' of $p$ and (pq) & & $\begin{array}{l}\text { zeroth } \\
\text { order }\end{array}$ & $\begin{array}{l}\text { first } \\
\text { order }\end{array}$ & $\begin{array}{l}\text { eocond } \\
\text { order }\end{array}$ & $\begin{array}{c}10 t \\
\Gamma_{p}+r_{(m)}\end{array}$ \\
\hline
\end{tabular}
equivalent coordinates of type $p$. The quantities $N_{(m)}$ and $\Gamma_{(m)}$ are similar to the diagonal terms except that the one with the subscript $(p q)$ refer to an interaction of a particular type between a valence coordinate of type $p$ and another of type $q$. There can be more than one type of interaction between a given set of coordinate types, $\mathrm{p}$ and $\mathrm{q}$. For instance, a C-H stretch and an $\mathrm{H}-\mathrm{C}-\mathrm{H}$ bend may share a terminal $\mathrm{H}$-atom in one type of interaction, or share the $\mathrm{C}$-atom in another interaction type, or share the $\mathrm{C}-\mathrm{H}$ bond in still another. Table 6 is an example of the additivity parameters, $\Gamma_{p}$ and $\Gamma_{(\mathbf{A})}$, for $n$-alkanes.

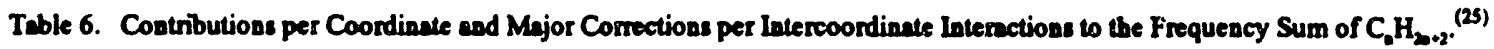

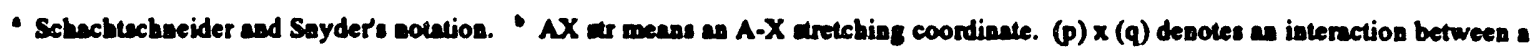
coordinate of type $p$ and a coordinate of type $q$, and apecification for a particula kind of interaction is given after a cemicolon. ' $N_{1}=$ aumber of coordinutes of king $p . N_{(w)}=$ aumber in intercoordinate intenaction of kind (pq). The constraints are given in the parentheses. - $\Gamma_{p}$ is the contribution to the frequency aum per coordinate of type $p$, expreased is $\mathrm{cm}^{-1} \Gamma_{(\mathrm{mov}}$ is the

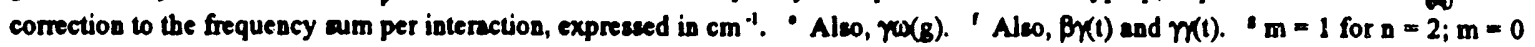
for $\mathbf{2} 23$. 
As Table 7 illustrates, these parameters yield the results which are within $0.7 \%$ of exact values of ZPE.

Table 7. Approximntions for the Frequency Sums of n-Alkanes ${ }^{\text {(25) }}$

\begin{tabular}{|c|c|c|c|}
\hline \multirow[b]{3}{*}{ n } & \multirow{3}{*}{$\begin{array}{l}\text { Exact } \\
\mathrm{cm}^{-1}\end{array}$} & \multicolumn{2}{|c|}{ Additivity } \\
\hline & & Value & \\
\hline & & $\mathrm{sm}^{-1}$ & \%erno \\
\hline 2 & $\overline{31,487}$ & $\overline{31,475}$ & -0.038 \\
\hline 3 & 43,677 & 43,395 & -0.65 \\
\hline 4 & 55,809 & 55,429 & -0.68 \\
\hline 5 & 67,917 & 67,435 & -0.71 \\
\hline 6 & 80,011 & 79,442 & -0.71 \\
\hline 7 & 92,101 & 91,448 & -0.71 \\
\hline 8 & 104,187 & 103,454 & -0.70 \\
\hline 9 & 116,271 & 115,469 & -0.69 \\
\hline 10 & 128,354 & 127,467 & -0.69 \\
\hline
\end{tabular}

Several simpler additivity rules of various precisions were also derived starting with Eqs. (99) and (111). The table of additivity parameters were also constructed for alkyl chlorides. ${ }^{(25)}$

\section{III-A-5. Other Applications}

The expressions for ZPE and $\delta(Z P E)$ presented here formed a basis for various correlation studies. The following is an account of these studies.

\section{Valence Coordinates and Hydrogeon Isotope Effects in $\mathrm{ZPE}^{(20)}$}

From Eq. (85), we have for the isotopic difference in the frequency sums,

$$
\delta \Sigma \omega_{1} \cong \beta_{1}^{\prime} \delta \Sigma \lambda_{1}+\beta_{2}^{\prime} \delta \Sigma \lambda_{1}^{2} \equiv \beta_{1}^{\prime} S_{1}+\beta_{2}^{\prime} S_{2},
$$

where $\beta_{1}^{\prime}$ and $\beta_{2}^{\prime}$ are related to $b_{1}$ and $b_{2}$ in the obvious manner, and $S_{1}$ and $S_{2}$ represent the sums of the first-order and second-order contributions, respectively.

Using the theory for the ZPE-additivity reported above, the isotope shift in the frequency sum can also be expressed as a sum of contributions of the valence coordinates. Thus,

and

$$
S_{1}=\Sigma_{p} T_{1}(p)+\Sigma_{(p)} T_{1}(p q) \quad \text {, }
$$

in which $T_{1}(p q)$ is the sum of all terms of the form $2 f_{i j} \delta g_{i j}$ where $i$ is a coordinate of type $p$, and $j$ a coordinate of type $q$, while $T_{2}(p q)$ is the sum of all second-order interaction terms, $T_{2}{ }^{m}(p q)$, each containing different types of geometric factor as in Eq. (27). Although we can conceive of various additivity formula for $\delta \Sigma \omega_{i}$, the best one that gave the physical insight without becoming too cumbersome and without losing the precision is

$$
\delta \Sigma \omega_{i} \cong S_{1}+\Sigma_{p} T_{2}^{1}(p)=S_{1}+\sum_{p} \eta_{p} f_{p}^{2} \delta g_{p}^{2}
$$


where $n_{p}, f_{p}$, and $g_{p}$ are the number of type-p coordinates and the diagonal elements of $F$ - and G-matrices, respectively. The precision is $0.7 \%$ or better. This is illustrated by Table $8{ }^{(26)}$ for $d_{1} / d_{0}$-substitution in methane.

Table 8. Mnjor Contibutions to leotope Shift in the Frequency Sum for $\mathrm{CH}_{3} \mathrm{D} / \mathrm{CH}_{4}{ }^{(\omega)}$

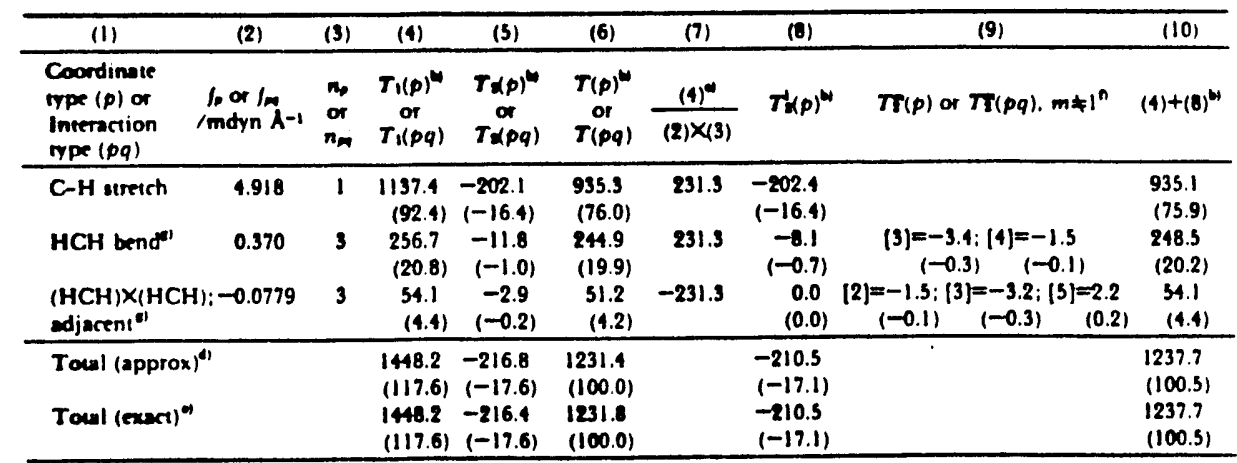

- F-matrix taken from Hartsborn and Shiner. (12) The approximation of $\mathrm{Case} C 1$ is used; $k=0.77, \xi=3.614329, \eta=1.274406$, $\omega=3022.31 \mathrm{~cm}^{-1}$ and $\delta \Sigma \omega_{(}(\mathrm{exect})=1241.45 \mathrm{~cm}^{-1}$. - All $\mathrm{T}$ terms are in $\mathrm{cm}^{-1}$. The numbers in parentheses below the wavenumbe values are percent of $\delta \Sigma \omega_{(}(e x a c t)$. " In units of $A$ mdyn ${ }^{-1} \mathrm{~cm}^{-1}$. " The aum of the contributions tabulated. - The aum of all contributions, both tabulated and non-tabulated. Contributions whose abeolute magnitudes are less than $0.1 \%$ of $\delta \Sigma \omega_{\text {. }}(e x a c t)$ are not tabulated. 'Number in the brackets is the type of intenction, $m$. - All bending coordinates are weiglted by the equilibrium $\mathrm{C}$-H bond length. The adjacent bends are two beads that share a C.H bond.

\section{Classification of Molecules According to 8(ZPE) for H/D-Isotope Effect ${ }^{(27)}$}

Although all H-X stretching coordinates are nearly independent of other vibrations in the molecule, $\delta(Z P E)$ is still affected by the molecular geometry near the site of the H/D substitution. A classification presented in Table 9 facilitated a finer tuning for a higher precision and a better correlation of the isotope effect. The basis of Table 9 is a study of a few dozens of hydrogeneous molecules. In the Table, $Y$ generally represents a molecular moiety rather than an atom. 
Table 9. Chasification of Molecules for H/D Isotope Shifts in Zero-Point energyies ${ }^{\omega}$

\begin{tabular}{|c|c|c|c|c|c|c|c|}
\hline \multirow{2}{*}{ Group } & \multirow[b]{2}{*}{ Description } & \multirow[b]{2}{*}{$\left.\boldsymbol{x}_{0}\right)$} & \multicolumn{5}{|c|}{ Reprewentative molecule } \\
\hline & & & molecule & pair & $k_{0}$ & $\begin{array}{l}\% \text { error } \\
\text { at } k_{n}\end{array}$ & Ref. \\
\hline In & $\begin{array}{l}\mathrm{HX}-\mathrm{Y} \text { [Linear] } \\
\mathrm{HX}-\mathrm{Y}-\mathrm{Z} \text { [Linear]: }\end{array}$ & 0.277 & $\mathrm{HCN}$ & $d_{0} / d_{1}$ & 0.259 & -0.72 & 4 \\
\hline Ibl & $(Z=H)$ & 0.264 & $\mathrm{C}_{2} \mathrm{H}_{1}$ & $\begin{array}{l}d_{0} / d_{1} \\
d_{2} / d_{1}\end{array}$ & $\begin{array}{l}0.266 \\
0.262\end{array}$ & $\begin{array}{r}0.07 \\
-0.07\end{array}$ & 5 \\
\hline Ib2 & $(Z \neq H)$ & 0.197 & HCCF & $d_{0} / d_{2}$ & 0.206 & 0.37 & 3 \\
\hline Ic & HX $-\mathbf{Y}$ [Bent] & 0.427 & $\mathrm{HOCl}$ & $d_{0} / d_{1}$ & 0.442 & 0.40 & 6 \\
\hline Id & HX...(Y) [Planar] & 0.278 & $\mathbf{C}_{\mathbf{8}} \mathbf{H}_{\mathbf{0}}$ & $d_{0} / d_{2}$ & 0.269 & -0.35 & 7 \\
\hline \multirow[t]{3}{*}{ Ie } & $H X \cdots(Y)$ [Nonplanar] & $\begin{array}{c}0.322 \\
(0.278)\end{array}$ & CHF, & $d_{0} / d_{1}$ & 0.409 & 2.78 & 8 \\
\hline & & & $\mathrm{CHCl}_{2}$ & $d_{0} / d_{3}$ & 0.299 & $\begin{array}{l}-0.90 \\
(0.86)^{n}\end{array}$ & 5 \\
\hline & & & $\mathrm{CHBr}_{1}$ & $d_{0} / d_{1}$ & 0.257 & $\begin{array}{l}-2.36 \\
(-0.84)^{n}\end{array}$ & $\mathbf{s}$ \\
\hline II: & $\mathrm{H}_{2} \mathrm{X}$ & 1.052 & $\mathrm{H}_{2} \mathrm{O}$ & $d_{0} / d_{3}$ & 1.005 & -0.28 & 9 \\
\hline IIb & $\mathrm{H}, \mathrm{X}-\mathrm{Y}$ [Planar] & 0.718 & $\mathrm{H}_{2} \mathrm{CO}$ & $d_{0} / d_{1}$ & 0.742 & 0.31 & 10 \\
\hline IIc & $\begin{array}{l}\mathrm{H}_{2} \mathrm{XY}_{2} \text { [Nonplanar] } \\
\mathrm{H}_{1} \mathrm{X}-\mathrm{YZ}_{1} \text { [Planar]: }\end{array}$ & 0.441 & $\mathrm{CH}_{3} \mathrm{~F}_{2}$ & $d_{0} / d_{2}$ & 0.498 & 1.38 & 11 \\
\hline IIdI & $(\mathrm{Z}=\mathrm{H})$ & 0.398 & $\mathrm{CH}_{2} \mathrm{CH}_{3}$ & $\mathrm{CH}_{2} / \mathrm{CD}_{2}$ & 0.401 & 0.08 & 12 \\
\hline IId2 & $(Z \neq H)$ & 0.272 & $\mathrm{CH}_{2} \mathrm{CF}_{2}$ & $d_{0} / d_{2}$ & 0.276 & 0.16 & 5 \\
\hline IIe & $H, X \ldots(Y)$ [Nonplenar]r) & 0.400 & $\begin{array}{l}\mathrm{CH}_{2} \mathrm{CH}_{2} \mathrm{Cl} \\
\mathrm{CH}_{2} \mathrm{CH}_{2} \mathrm{CH}_{2}\end{array}$ & $\begin{array}{l}\mathrm{d}_{0} / \mathrm{d}_{2} \\
\mathrm{CH}_{2} / \mathrm{CD}_{1}\end{array}$ & $\begin{array}{l}0.390 \\
0.410\end{array}$ & $\begin{array}{r}-0.30 \\
0.26\end{array}$ & $\begin{array}{l}5 \\
5\end{array}$ \\
\hline III. & $\mathrm{H}, \mathrm{X}$ & 0.723 & $\mathrm{NH}_{3}$ & $d_{0} / d_{1}$ & 0.677 & -0.64 & 13 \\
\hline \multirow[t]{4}{*}{ IIIb } & $H, X-Y$ [Nonplanar]d) & $\begin{array}{l}0.462 \\
(0.437)^{\bullet)}\end{array}$ & $\mathrm{CH}_{3} \mathrm{~F}$ & $d_{0} / d_{2}$ & 0.337 & 1.69 & 14 \\
\hline & & & $\mathrm{CH}_{2} \mathrm{Cl}$ & $d_{0} / d_{8}$ & 0.463 & $\begin{array}{l}0.46 \\
(0.63)^{n}\end{array}$ & 5 \\
\hline & & & $\mathrm{CH}_{\mathrm{B}} \mathrm{Br}$ & $d_{0} / d_{2}$ & 0.432 & $\begin{array}{l}-0.76 \\
(-0.13)^{n}\end{array}$ & 5 \\
\hline & & & $\mathrm{CH}_{8} \mathrm{I}$ & $d_{0} / d_{2}$ & 0.415 & $\begin{array}{l}-1.21 \\
(-0.58)^{n}\end{array}$ & 5 \\
\hline IIIc & $\begin{array}{l}H_{1} X-Y-Z \cdots(W) \\
{[X-Y-Z \text { Linear }]}\end{array}$ & 0.528 & $\mathrm{CH}_{\mathbf{2}} \mathrm{CCH}$ & $d_{0} / d_{0}$ & 0.574 & 0.91 & 5 \\
\hline IIId & $\begin{array}{l}H_{2} X-Y \cdots(Z) \\
\quad \text { (Around } Y \text { nonlinear] })\end{array}$ & 0.419 & $\begin{array}{l}\mathrm{CH}_{2} \mathrm{CH}_{2} \mathrm{Cl} \\
\mathrm{CH}_{2} \mathrm{CH}_{2} \mathrm{CH},\end{array}$ & $\begin{array}{l}\mathrm{CH}_{2} / \mathrm{CD}_{1} \\
\mathrm{CH}_{2} / \mathrm{CD}_{2}\end{array}$ & $\begin{array}{l}0.436 \\
0.442\end{array}$ & $\begin{array}{l}0.46 \\
0.63\end{array}$ & $\begin{array}{l}5 \\
5\end{array}$ \\
\hline IV & $H_{6} \mathrm{X}$ & 0.686 & $\mathrm{CH}_{4}$ & $\begin{array}{l}d_{0} / d_{1} \\
d_{0} / d_{1}\end{array}$ & $\begin{array}{l}0.733 \\
0.646\end{array}$ & $\begin{array}{r}0.64 \\
-0.59\end{array}$ & 15 \\
\hline
\end{tabular}

\footnotetext{
- Case-B spproximution. - The group avenge of the optimumk. " The fird-named isotopic species is the reference molecules from which the $\lambda$ was obtained. - With no possibility of torsion involving the $H$ atom being subatituted. - Alternate $k_{0}$ value obtained by excluding fluoro-compounds. The numbers in the parentheses are the pencent errors oblained by using the altemale $k_{0}$ listed in the parentheses in the third column. "With possibility of torrions involving the $\mathrm{H}$ alom being aubatituted.
} 


\section{$\delta(Z P E)$ in Hydrides and Electronegativity ${ }^{(29,33)}$}

As expected from the additivity for $\delta(Z P E)$, a linear relationship was found between the square root of A-H stretching force constants in $\mathrm{AH}_{n}(\mathrm{n}=1 \sim 4)$ molecules and $\delta(\mathrm{ZPE})$ for H/D-substitution. ${ }^{(29)}$ Figure 6 demonstrates the fact that the contributions of $f_{i j}(i=$ stretch) to $\delta(Z P E)$ through all interaction terms such as $f_{i j} f_{i j} \delta\left(g_{i} g_{i j}\right)$ and $f_{i j} f_{j j} \delta\left(g_{i j}{ }^{2}\right)$ are negligible. A similar plot, Figure 7, for the H-A-H bending contributions is more scattered, reflecting some significant contributions by these interaction terms.

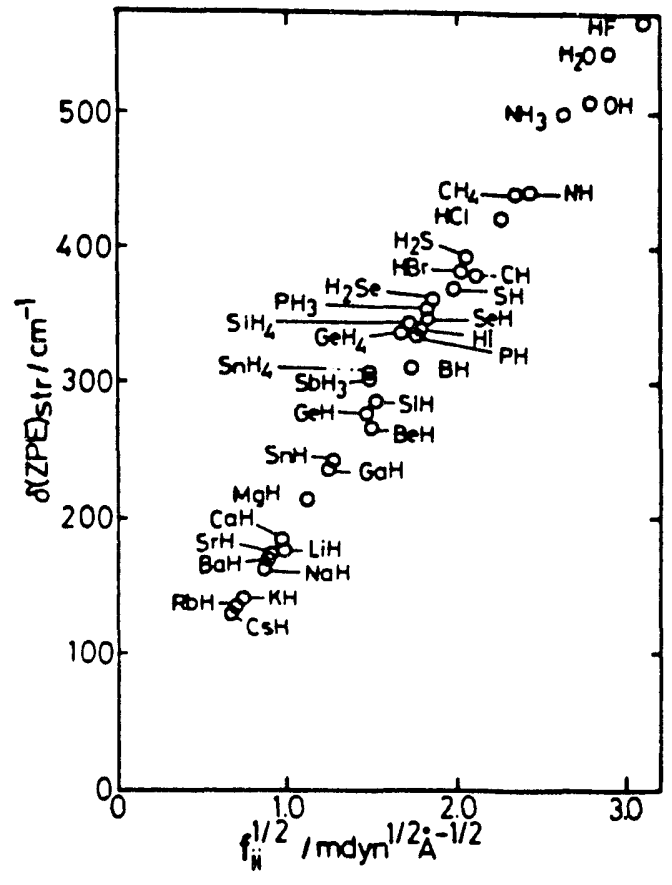

Figure 6. Correlation between aretching force constant and its contribution to \&(ZPE).

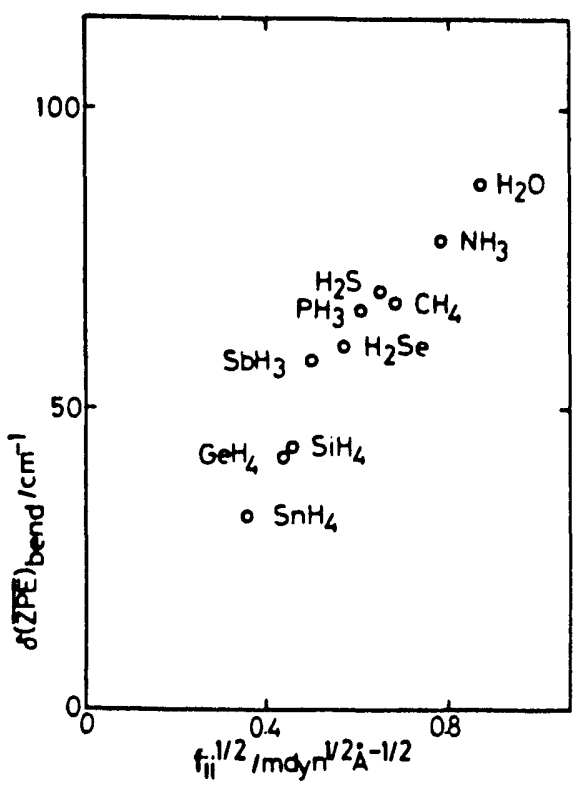

Figure 7. Comelation between bending force constant and its contribution to 8 (ZPE).

Since the first introduction of the concept of electronegativity by Pauling ${ }^{(12)}$ in 1930's, various attempts have been made for improved definitions of electronegativity. Among them are; ones by Wilmshurst $\left(\chi_{W}\right)^{(129,134)}$, by Inomata and Masuda $\left(\chi_{1}\right)^{(130)}$, and by Boyd and Edgecome $\left(\chi_{B}\right)^{(131)}$, and the unshielded core potential $\left(V_{B}\right)$ as a measure of electronegativity by Luo and Benson. ${ }^{(132)}$ Gordy's scale is based on numbers of electrons in the valence shells and the single bond covalent radii of the atoms. ${ }^{(133)}$ Wilmshurst extended Gordy's formula to include the electronegativity calculations for various molecular 
moieties. Boyd and Edgecombe obtained their atomic and group electronegativity values on the basis of the topological properties of the electron density distribution in molecules.

It can be shown ${ }^{(33)}$ that the contribution of $\mathrm{H}-\mathrm{X}$ stretching motions to $\delta(\mathrm{ZPE})$ increases linearly with the electronegativity of $X$, where $X$ is either an atom or a group of atoms. Wilmshurst's $\chi_{w}$ is

$$
x_{w}=g\left(1+m_{H} / m_{x}\right)^{\cdot 1 / 2} v_{w}-h \quad,
$$

where $m_{\alpha}$ is the mass of atom $\alpha$, and $g$ and $h$ are constant property of $X$. No isotope effects were considered by these authors. However, since all electronegativities must be isotope-independent in accordance with Borm-Oppenheiner principle, the parameter $\mathbf{g}$ must be a function of isotopic mass in such a way to cancel out the isotope dependence of the factors, $\left(1+m_{H} / m_{x}\right)^{-1 / 2}$ and $v_{w}$. If one assumes that the bond, $\mathrm{H}-\mathrm{X}$, in a polyatomic environment are independent harmonic oscillator (which assumption we know is a good one, as we have discussed throughout Sections II-A and II-B of the present report), the parameter $\mathrm{g}$ for the H/D isotope effects must be in a form,

$$
g=g_{0} \sqrt{m_{H}} \text { or } \quad=g_{0} \sqrt{m_{D}} \text {, }
$$

while $h$ is independent of isotop: substitutions. With this modification of $\chi_{w}$, the stretching frequency shift, $\delta v$, must have a linear dependence on the electronegativity. Thus, for the $D / H$-isotope effect, we have

$$
\delta(Z P E)_{-}=r \chi+s
$$

Table 10 summarizes our comparison, in which

$$
\delta(Z P E) \cong \delta(Z P E)_{\text {ou }}+\delta(Z P E)_{\text {mad }}
$$

Figure 8 illustrates a linearity for each main group, IVA, VA, VIA, and VIIA, of the periodic table. The average deviation from the linear line is better than several wavenumbers. 
Table 10. Composents of \&(ZPE) esd eloctrosegativity

\begin{tabular}{|c|c|c|c|c|c|c|c|c|c|}
\hline Nolecule & $8(Z P E)$ & $\delta(Z P E)_{01}$ & $\delta(Z P E)_{0 . n A}$ & CORR & $\begin{array}{l}\text { Atom or } \\
\text { growp }\end{array}$ & $x_{v}$ & $x_{1}$ & $x:$ & $V_{b}$ \\
\hline "....... & (........ & и... & 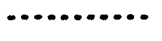 & $\cdots \cdots$ & ......... & $\cdots$ & $\cdots$ & $\cdots$ & $\cdots$ \\
\hline uF & 568.9 & 568.9 & & & $F$ & 3.94 & 3.89 & 4.00 & 9.815 \\
\hline $\mathrm{NCl}$ & 122.9 & 422.8 & & & $\mathrm{Cl}$ & 3.00 & 3.00 & 3.05 & 7.04 \\
\hline$M B r$ & 381.8 & 381.8 & & & $B r$ & 2.68 & 2.68 & 2.75 & 6.13 \\
\hline 1 & 334.6 & 334.6 & & & 1 & 2.38 & 2.38 & & 5.25 \\
\hline$N_{2} O$ & 632.5 & 545.8 & 87.5 & -0.9 & $-\mathrm{OH}$ & 3.86 & 3.72 & 3.64 & 8.11 \\
\hline$n_{2} S$ & 463.3 & 393.8 & 60.5 & 0.0 & $-S H$ & 2.61 & 2.66 & 2.63 & 5.77 \\
\hline $\mathrm{N}_{2} \mathrm{Se}$ & 407.6 & 349.5 & 69.2 & -11.1 & -SeH & 2.37 & 2.36 & 2.47 & 5.13 \\
\hline $\mathrm{NH}_{3}$ & 659.0 & 500.0 & 156.7 & 2.3 & $-\mathrm{HH}_{2}$ & 3.40 & 3.02 & 3.10 & 6.67 \\
\hline$P H_{2}$ & 485.0 & 355.7 & 133.0 & -3.7 & $\cdot P H_{2}$ & 2.20 & 2.19 & 2.17 & 4.55 \\
\hline Asth & 450.4 & 338.0 & 156.7 & -44.3 & $-\mathrm{AsH}_{2}$ & 2.03 & 2.05 & 2.26 & 4.20 \\
\hline SbH, & 396.4 & 304.6 & 116.3 & -24.5 & $-\mathrm{SbH}_{2}$ & 1.78 & 1.86 & & 3.55 \\
\hline $\mathrm{CH}_{4}$ & 645.4 & 440.4 & 203.7 & 1.3 & $-\mathrm{CH}_{3}$ & 2.63 & 2.47 & 2.58 & 5.19 \\
\hline $\mathrm{SiH}_{4}$ & 474.3 & 343.0 & 131.5 & -0.2 & $-\mathrm{SiH}_{2}$ & 1.78 & 1.91 & 1.91 & 3.41 \\
\hline $\mathrm{CeH}_{4}$ & 464.3 & 337.8 & 127.2 & -0.7 & - CelH3 & 1.60 & 1.88 & 2.05 & 3.24 \\
\hline $\mathrm{SnH}_{4}$ & 405.0 & 308.0 & 97.8 & -0.8 & $-\mathrm{SnH}_{2}$ & 1.51 & 1.71 & & 2.83 \\
\hline
\end{tabular}

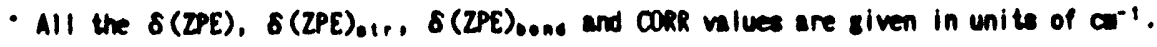

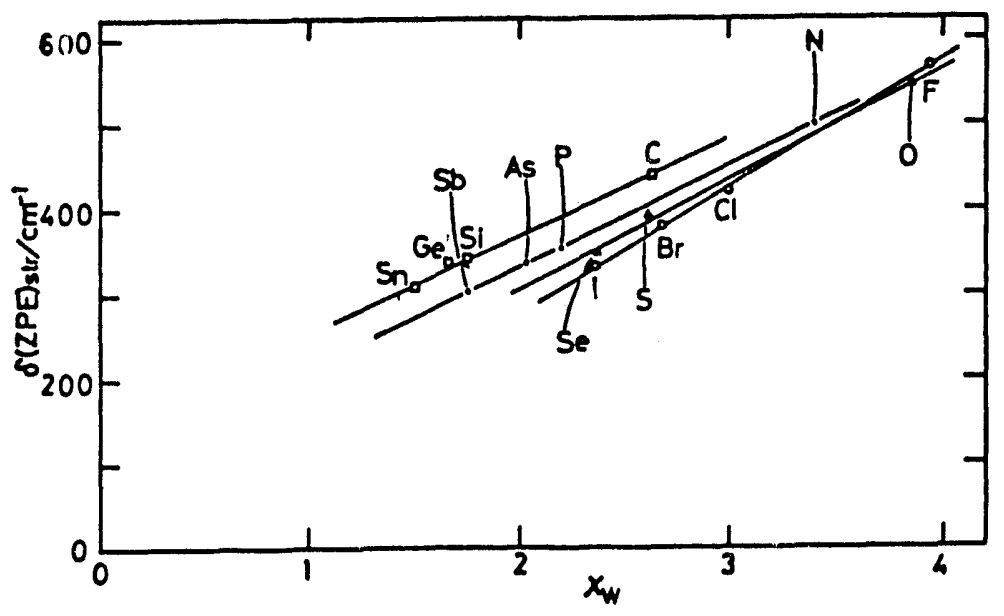

Figure 8. Plot of \&(ZPE) vs. Wilmshura's electronegativity 


\section{III-B. Isotope Effects in Zero-Point Energy Shifts upen Condensation}

One of the most important factors that determine the isotopic difference in the vapor pressures is the isotope shift in the ZPE upon condensation of gaseous molecules, $\delta \Delta(Z P E)$;

$$
\begin{aligned}
& \delta \Delta(\mathrm{ZPE})=\frac{1}{2} \mathrm{hc} \sum_{1}^{f}\left[\delta \omega_{1}(\mathrm{~g})-\delta \omega_{1}(\mathrm{c})\right], \\
& \text { and } \quad \Delta \delta(\mathrm{ZPE})=\frac{1}{2} \mathrm{hc} \sum_{i}^{f}\left[\Delta \omega_{i}{ }^{\prime}-\Delta \omega_{i}\right] \text {. } \\
& \text { where } \quad \delta \omega_{i}=\omega_{i}{ }^{\prime}-\omega_{i} \\
& \text { and } \Delta \omega_{i}=\omega_{1,00}-\omega_{i, \text { ondened }} \text {, } \\
& \text { so that } \delta \Delta(Z P E)=\Delta \delta(Z P E)
\end{aligned}
$$

Ordinarily, $\delta \Delta(Z P E)$ as defined here is positive. See Figure 9 . For the condensed phase systems in which the only intermolecular force is Lodon dispersion forces, the sum of the condensation shifts in the vibrational eigenvalues, $\Delta \lambda_{\text {in }}$, of a representative molecule $A$ is

$$
\sum_{i}^{f} \Delta \lambda_{i A}=-2 \frac{\alpha_{B}}{S_{A B}^{6}} \sum_{i}^{f}\left(\frac{\partial \vec{P}}{\partial Q_{i A}}\right)^{2}
$$

where $\alpha_{B}$ is the electronic polarizability of a neighbor molecule $B$ in its ground state, $S_{A B}$ is the equilibrium average intermolecular separation in the cusidensed phase, and $\left(\partial \vec{P} / \partial Q_{W}\right)$ is the first derivative of the dipole moment vector $P$ with respect to the $i$-th normal coordinate $Q_{i}$ of the representative molecule. ${ }^{(124)}$

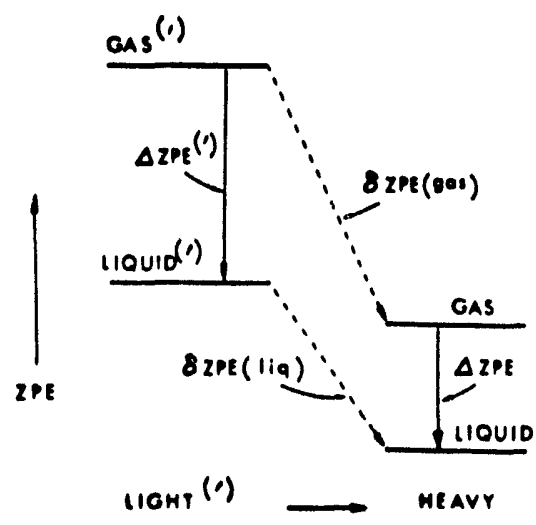

Figure 9. Isotope effect on ZPE uhif upon condenation. ${ }^{(13)}$

A combination of Eqs. (120) and (123) with a $\delta(Z P E)$ approximation such as Eqs. (111), (112) and (115) provides a direct means of evaluating $\delta \triangle(Z P E)$ using properties of the gaseous molecule. At the time of our study of this problem, the only available relationship between $\Sigma \omega_{1}$ and $\Sigma \lambda_{1}$ was that of Bigeleisen and Goldstein. ${ }^{(125)}$ The first term of this infinite series is

$$
\sum_{i}^{f} \delta \omega_{i} \cong \frac{1}{8 \pi^{2} c^{2}} \frac{1}{\omega^{2}} \sum_{i}^{f} \delta \lambda_{i}
$$

so that the vapor pressure isotope effect parameter, $B=\delta \Delta(Z P E)$, becomes

$$
B \cong \frac{1}{8 x^{2}} \frac{h}{c k}\left(\frac{c}{\omega_{0}}\right)\left(\frac{\alpha}{s^{r}}\right) \Sigma_{i}\left[\left(\frac{\partial \vec{p}}{\partial Q_{1}^{\prime}}\right)^{2}-\left(\frac{\partial \vec{P}}{\partial Q_{1}}\right)^{2}\right]_{\text {gas }} \text {. }
$$


where $C$ is a condensed-phase structural constant which accounts for an overall influence of all molecules in the first and outer coordination spheres and is well known for various crystal lattice structures.

The magnitude of $\left(\partial \mathrm{P} / \partial \mathrm{Q}_{1}\right)^{2}$ can be evaluated by transforming the polar tensor,

$$
\vec{P}_{x}(\alpha)=\left(\begin{array}{lll}
\partial P_{x} / \partial X_{\alpha} & \partial P_{x} / \partial Y_{\alpha} & \partial P_{x} / \partial Z_{\alpha} \\
\partial P_{y} / \partial X_{\alpha} & \partial P_{y} / \partial Y_{\alpha} & \partial P_{y} / \partial Z_{\alpha} \\
\partial P_{z} / \partial X_{\alpha} & \partial P_{z} / \partial Y_{\alpha} & \partial P_{z} / \partial Z_{\alpha}
\end{array}\right)
$$

into the center-of-mass/ principal axis coordimate system. The partial derivatives in Eq. (126) are the cartesian components of the derivatives for atom $\alpha$. Then, it can be shown ${ }^{(13)}$ that

$$
\left(\frac{\partial \vec{P}}{\partial Q_{i}}\right)^{2}=\sum_{\alpha}^{N} \mu_{\alpha} \xi_{\alpha}^{2}-\Omega-2 D .
$$

where $\mu_{a}$ is the reciprocal mass of atom $\alpha$, and $\xi_{\alpha}$ is the effective atomic charge on atom $\alpha$ as defined by Person and Newton, ${ }^{(12 \pi)}$

$$
\xi_{\alpha}^{2}=\operatorname{Tr}\left[\vec{P}_{x(\alpha)} \vec{P}_{x(\alpha)^{t}}\right]
$$

where $\vec{P}_{x}(\alpha)^{\prime}$ is the transpose matrix. The term $\Omega$ and $D$ are correction terms which, respectively, represent the effects of molecular rotations and external-internal kinetic energy interactions. The terms of $\vec{P}_{x}(\alpha)$ can be evaluated from a quantum mechanical algorithm.

By means of Eqs. (124)-(138), $\delta \Delta(Z P E)$ can be expressed as a product of an isotope-independent condensed-phase factor and an isotopic difference in the gas-phase properties. ${ }^{(13)}$ The theory was tested (13) by calculating the effective atomic charges for carbon and bydrogen in ethylene using the CNDO/2 molecular orbital program. The theory was applied to the $\mathrm{D} / \mathrm{H}-$ and ${ }^{13} \mathrm{C} /{ }^{12} \mathrm{C}$ - isotope effects in methane and fluoromethanes, and the results were compared with experimental values of the total IR absorption intensities and $\delta \Delta(Z P E)$. This led to a set of rules of thumb for predicting a large value of $\delta \Delta(Z P E){ }^{(13)}$

The following properties and features of a molecule generally increase $\delta \Delta(Z P E)$.

a) Highly polar bonds, because they increase $\xi_{a}{ }^{2}$. The effect is more marked for a central atom surrounded by polar bonds than for a terminal atom bonded to such a central atom. Thus, for a ${ }^{13} \mathrm{C} /{ }^{12} \mathrm{C}$ substitution, the $\mathrm{B}$ factor for $\mathrm{CF}_{4}$ should be greater than that for $\mathrm{CH}_{4}$.

b) Larger difference in the reciprocal masses of the substituted isotopic atoms.

c) Molecular properties that make $\delta \Omega$ smaller, which include smaller electric dipole moments of the molecule, larger principal moments of inertia, and central isotope substitution rather than peripherial isotope substitution. 
d) Absence of hydrogen atoms, which, for instance, makes the Bigeleisen-Goldstein characteristic frequency $\omega_{0}$ smaller.

e) More closely packed liquid structure, or smaller liquid molal volume, which tends to increase the effective value of the Lennard-Jones and Ingham crystal constant and to decrease the nearest-neighbor distances of liquid molecules.

As presented in Section IV below, the results of our studies of VPIE of fluoromethanes qualitatively agree with these general rules. 


\section{Vapor Pressure Isotope Effects}

The yapor pressure isotope effect (VPIE) is a useful tool for elucidating molecular forces in condensed phases. We studied the VPIE in the following specific areas:

a) VPIE of fluoromethanes, $\mathrm{CH}_{4 . .} \mathrm{F}_{\mathrm{a}}(\mathrm{n}=1 \sim 3)$ : References $(13,16,17,18,20,21,28)$

b) VPIE of ammonia: References $(31,30)$

c) Theories related to VPIE: References $(7,8,9,13)$

\section{IV-A. Vapor Pressure Isotope Effects in Fluoromethanes}

Vapor pressure isotope effects were measured using the precision cryostat ${ }^{(170)}$, which provides a computer-control for stability and uniformity $(< \pm 0.001 \mathrm{~K})$ of cryogenic temperatures $(3 \sim 300 \mathrm{~K})$ and for ease of operation. Table 11 lists the isotopic molecules and the temperature ranges that we studied..

Table 11. Fluoromethenes Studied

\begin{tabular}{|c|c|c|}
\hline Molegules & Jeotopes & Tempernture ninge \\
\hline Methyl Aluorides (liq) & ${ }^{12} \mathrm{CH}, \mathrm{F},{ }^{12} \mathrm{CD}, \mathrm{F},{ }^{1} \mathrm{CH}, \mathrm{F}$ & $132.48 \sim 213.12 \mathrm{~K}$ \\
\hline Metbylese difluorides (liq) & ${ }^{12} \mathrm{CH}_{2} \mathrm{~F}_{2},{ }^{12} \mathrm{CD}_{2} \mathrm{~F}_{2},{ }^{13} \mathrm{CH}_{2} \mathrm{~F}_{2}$ & $149.36 \sim 244.82 \mathrm{~K}$ \\
\hline Fluoroforms (liquid) & ${ }^{12} \mathrm{CHF}_{3},{ }^{12} \mathrm{CDF}_{3},{ }^{13} \mathrm{CHF}$, & $125.95 \sim 212.16 \mathrm{~K}$ \\
\hline
\end{tabular}

For each isotopic pair, the data on the vapor pressure $\mathrm{P}^{\prime}$ of ${ }^{12} \mathrm{CH}_{4}, \mathrm{~F}_{\mathrm{a}}$ taken as the reference, and $\delta \mathrm{P}$, the isotopic difference defined as $P^{\prime}-P$, were converted to the ratio of the RPFR of the condensed phase, $f_{c}$, to that of the gas phase, $f_{z}$, by a conversion;

$$
\ln \frac{f_{c}}{f_{B}}=\left[1+P^{\prime}\left(B_{0}-\frac{V_{c}}{R T}\right)\right] \ln \frac{P^{\prime}}{P}-\ln f_{\text {rot }},
$$


where $B_{0}$ and $V_{c}$, are, respectively, the second virial coefficient and the molal volume of the condensed phase, and $f_{\text {rat }}$ is non-classical rotational correction. ${ }^{(138)}$ Figures $10 \sim 15$ are plots of $T \ln \left(f_{c} / f_{\mathrm{a}}\right)$ against $T^{-1}$. The differences between in $\left(f_{c} / f_{e}\right)$ and in $\left(P^{\prime} / P\right)$ are mostly within 1 to $2 \%$ of each other.

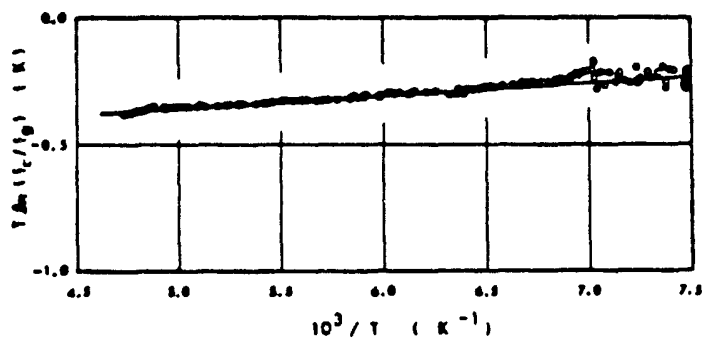

Figure 10. ${ }^{4} \mathrm{C} /{ }^{12} \mathrm{C}$ VPIE in methyl firoride ${ }^{013}$ : The wolid liee was computed from F-matrices of Tables 12 ind 13.

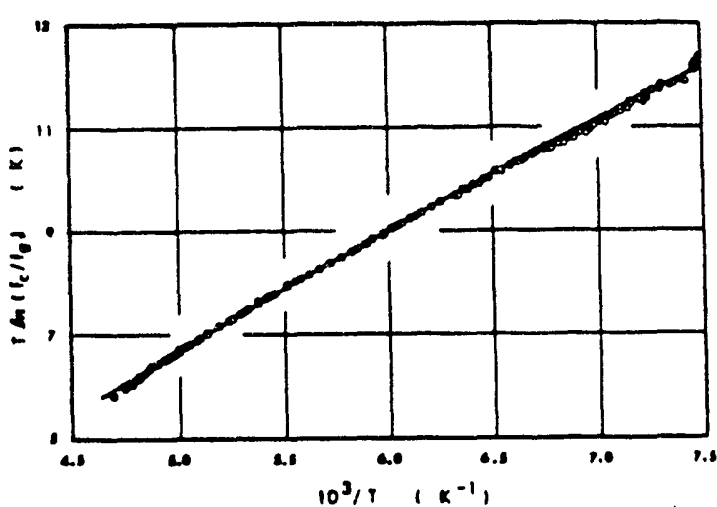

Figure 11. D/H VPIE in methyl fluoride ${ }^{\text {Q1): }}$ The solid line computed from F-matrices of Tables $12 \& 13$.

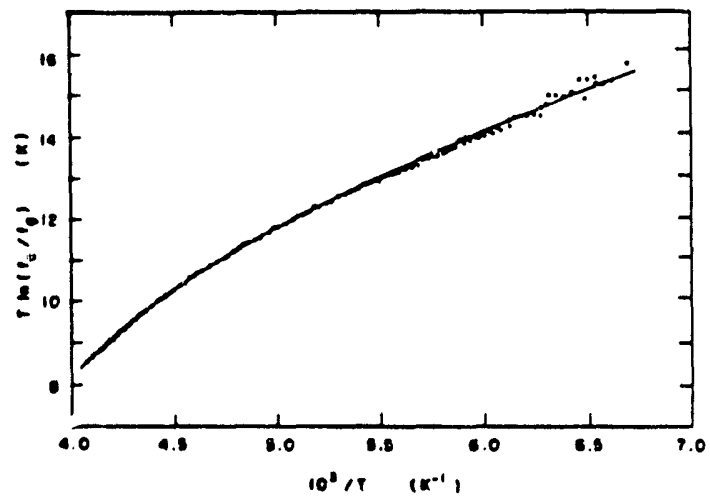

Figure 13. D/H VPIE in metbylene difluoride en: The solid line was computed from F-matrices of Tables 14 and 15.

Figure 16 summarizes the plots of Figures $10 \sim 15$ and compare them with the similar plots made from the data of VPIE's of methane. ${ }^{(136,137)}$ The following general observations are made.

1) All hydrogen isotope effects in the fluoromethanes are normal $\left(P^{\prime}>P\right)$, while the $D / H$ effects in methane are inverse $\left(\mathrm{P}^{\prime}<\mathrm{P}\right)$.

2) All carbon isotope effects in the fluoromethanes are inverse, while the carbon isotope effects in methane is normal. 


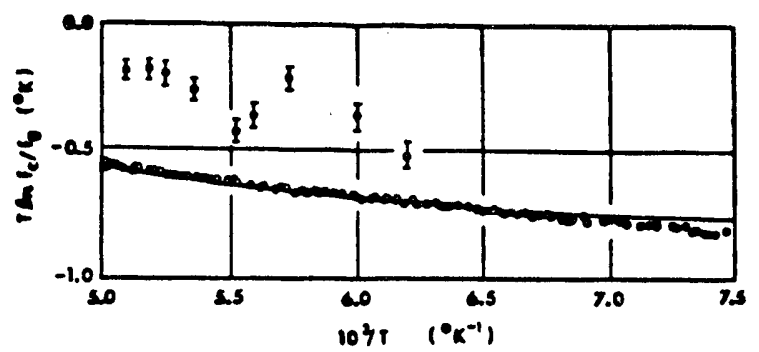

Figure 14. " $\mathrm{C} /{ }^{12} \mathrm{C}$ VPIE in fluoroform. ${ }^{\text {nh }}$ The solid line was computed from F-matrix of Tables 16 \&17.

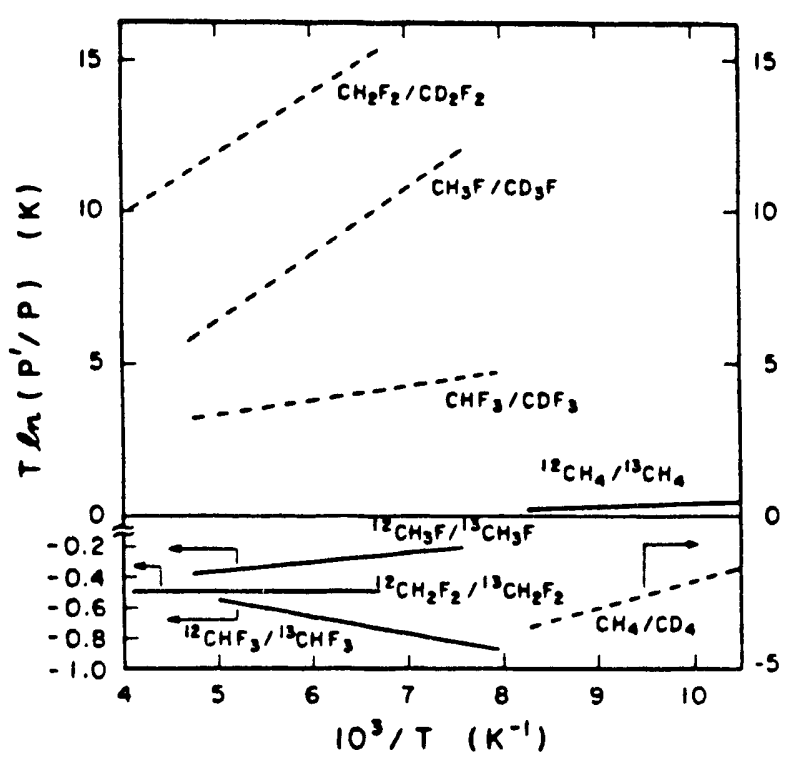

Figure 16. Comparieon of VPIE's in liquid fluoromethanes and liquid methane ${ }^{203}$ : Solid lines are ${ }^{13} \mathrm{C} /{ }^{12} \mathrm{C}$ effects; dashed lines are the effects of perdeuteriosubatitutions.

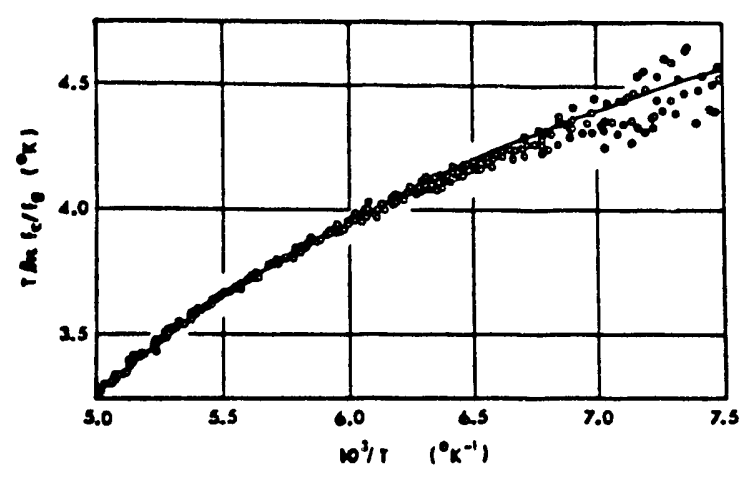

Figure 15. D/H VPIE in fluoroform. ${ }^{\text {(n) }}$ The eolid line was computed from F-matrix of Tables 16 and 17.

3) The carbon isotope effects in $\mathrm{CH}_{2} \mathrm{~F}_{2}$ are roughly of the same order of magnitude as the average of the carbon isotope effects in $\mathrm{CH}_{3} \mathrm{~F}$ and $\mathrm{CHF}_{3}$.

4) The $\mathrm{D} / \mathrm{H}$ effects in $\mathrm{CH}_{2} \mathrm{~F}_{2}$ is unusually large compared to the $\mathrm{D} / \mathrm{H}$ effects in the other fluoromethanes:

$$
\frac{1}{2}\left[T \ln \left(P^{\prime} / P\right)_{C H_{2} F_{2} / C D_{2} F_{2}}\right] \gg \frac{2}{3}\left[T \ln \left(P^{\prime} / P\right)_{C H_{3} F / C D_{3} F}\right]
$$

and

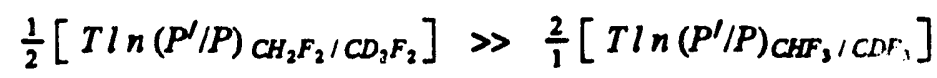

5) The curvature of every D/H-VPIE plot for the fluoromethanes is negative, while that of every ${ }^{13} \mathrm{C} /{ }^{12} \mathrm{C}$-VPIE in the fluoromethanes is positive. 
If each condensed phase molecule could be regarded as a point mass, then $\Delta \mathrm{H}_{\text {vap }}$ for the lighter isotopic molecule would be smaller than $\Delta \mathrm{H}_{v}$ of the heavier molecule, and one would have expected $\mathrm{P}^{\prime}$ $>P$. This is the normal VPIE. The external motion tends to lead to a normal VPIE. The inverse VPIE $\left(P^{\prime}<P\right)$ is due to the internal molecular structure and its vibrational motions that interact with the intermolecular forces in the condensed phase.

If all molecular forces were independent of temperature, then we would have the following as the simplest correlation between the RPFRs and the molecular parameters:

$$
\ln \left(f_{c} / f_{g}\right)=\sum_{i}^{\text {cuemal }} \delta \ln b\left(u_{i}\right)-\sum_{i}^{\text {intemal }} \Delta \delta \ln b\left(u_{i}\right) \equiv \frac{A}{T^{2}}-\frac{B}{T}
$$

where $b(u)$ is defined in Eq. (18a), $\delta \ln b(u)=\ln b\left(u^{\prime}\right)-\ln b(u), \Delta \delta \ln b(u)=\delta \Delta \ln b(u)$ as in Eqs. (120) (122), and

$$
\begin{aligned}
& A=\frac{1}{24}\left(\frac{h c}{k T}\right)^{2} \sum_{i}^{\text {Exemal }}\left(v_{i}^{\prime 2}-v_{i}^{2}\right)_{\text {condenced phase }} \\
& B=\frac{1}{2}\left(\frac{h c}{k T}\right) \sum_{i}^{\text {Intermal }}\left[\delta v_{i, \text { ges }}-\delta v_{i, \text { condosed }}\right]
\end{aligned}
$$

The term of $A T^{2}$ is the contribution of the external oscillatory motions of the whole molecule, which could be taken either as in the liquid simple cell model or as a central molecule in the liquid cluster model. The liquid cluster model will be presented in Section IV-C of this report.

As the temperature decreases, going from left toward right in Figures $10 \sim 16$, the liquids contract, the external forces increase, the magnitudes of the translational and rotational frequencies generally increase, and thus the factor A of Eqs. (132) and (133) would increase. The curvature of a plot of $T \ln \left(f_{c} / f_{e}\right)$ against $1 / T$ would then be positive.

The negative curvatures of the D/H-plots of Figures 11, 13, and 15 (Observation 5) thus imply dominant influence of the temperature on the B-term in Eq. (132): The B-factor should algebraically increase with the decreasing temperature in order for the curvature to be negative.

To quantitatively understand these phenomena, we conducted a series of normal coordinate analyses for the gaseous and liquid fluoromethanes. ${ }^{(17,21,23)}$ The best sets of F-matrices thus obtained are summarized in Tables $12 \sim 17$. The solid lines drawn through experimental points in Figures $10 \sim 15$ were calculated using these F-matrices. The T-dependence of the interaction force constants between the external and internal motions and the diagonal external elements for some of the external coordinates are necessary in order to reproduce the observed VPIE for both hydrogen and carbon substitutions, in particular, for good reproduction of;
a) the steep slopes and the negative curvature of the D/H-VPIE plots and the very small slope of the carbon isotope plots, and
b) the large normal VPIEs for the D/H substitutions and the small inverse VPIEs of the ${ }^{13} \mathrm{C} /{ }^{12} \mathrm{C}$ substitutions.


Table 12. F-matrix of Gacoous Methyl Fluoride el)

\begin{tabular}{|c|c|c|c|c|}
\hline \multicolumn{3}{|c|}{ diagonals } & \multicolumn{2}{|c|}{ off-diagonals } \\
\hline description & $\begin{array}{l}\text { nota- } \\
\text { tion }\end{array}$ & $\begin{array}{c}\text { value, } \\
\text { mdyn } \\
A^{-1}\end{array}$ & $\begin{array}{l}\text { nota. } \\
\text { tion }\end{array}$ & $\begin{array}{c}\text { value, } \\
\text { mdyn } \\
A^{-1}\end{array}$ \\
\hline $\begin{array}{l}\mathrm{C}-\mathrm{H} \text { stretch } \\
\mathrm{C}-\mathrm{F} \text { stretch } \\
\mathrm{HCH} \text { bend } \\
\mathrm{HCF} \text { bend }\end{array}$ & $\begin{array}{l}f_{d} \\
f_{D} \\
f_{a} \\
f_{\beta}\end{array}$ & $\begin{array}{l}4.686 \\
5.662 \\
0.408 \\
0.617\end{array}$ & $\begin{array}{l}f_{d d} \\
f_{d D} \\
f_{d \alpha}^{\prime} \\
f_{d \alpha} \\
f_{D \alpha} \\
f_{\alpha \alpha} \\
f_{\alpha \beta}^{\prime} \\
f_{d \beta} \\
f_{D \beta} \\
f_{\alpha \beta}^{\prime} \\
f_{\alpha \beta} \\
f_{\beta \beta}\end{array}$ & $\begin{array}{r}-0.036 \\
0.002 \\
-0.125 \\
-0.225 \\
-0.258 \\
-0.054 \\
0.118 \\
0.015 \\
0.258 \\
-0.143 \\
-0.118 \\
-0.144\end{array}$ \\
\hline
\end{tabular}

- All bending coordinates are weighted by the equilibri um $\mathrm{C}-\mathrm{H}$ bond length. An F.matrix element with one subscript is a diagonal element. When two off-diagonal elements are listed to a similar interaction, e.g., $f^{\prime}$ da and $f_{d a}$, the one without a prime refers to the interaction between two coordinates which share a common $\mathrm{C}-\mathrm{H}$ bond, while the one with a prime refers to the interaction between two coordinates which do not share a common $\mathrm{C}-\mathrm{H}$ bond.

Table 14. F-mutrix of Gaseous Methylene Difluoride an

\begin{tabular}{|c|c|c|c|c|c|}
\hline \multicolumn{3}{|c|}{ diagonals } & \multicolumn{3}{|c|}{ of-diagonals } \\
\hline description & nolation & $\begin{array}{c}\text { value, } \\
\text { mdyn } \\
\boldsymbol{A}^{-1}\end{array}$ & notation & & $\begin{array}{c}\text { value, } \\
\text { mdyn } \\
A^{-1}\end{array}$ \\
\hline $\begin{array}{l}\mathrm{C}-\mathrm{H} \text { stretch } \\
\mathrm{C}-\mathrm{F} \text { stretch } \\
\mathrm{HCH} \text { bend } \\
\text { FCF bend } \\
\mathrm{HCF} \text { bend }\end{array}$ & $\begin{array}{l}f_{d} \\
f_{0} \\
f_{0} \\
f_{0}\end{array}$ & $\begin{array}{l}4.829 \\
6.020 \\
0.462 \\
1.000 \\
0.635\end{array}$ & 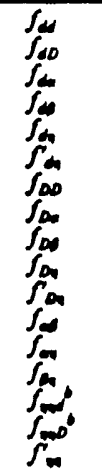 & $\begin{array}{l}\text { C-H, C-H } \\
\text { C-H, C-F } \\
\text { C-H, HCH } \\
\text { C-H, FCF } \\
\text { C-H, HCF } \\
\text { C-H, HCF } \\
\text { C-F, C-F } \\
\text { C-F, HCH } \\
\text { C-F, FCF } \\
\text { C-F, HCH } \\
\text { C-F, HCF } \\
\text { HCH, FCF } \\
\text { HCH, HCF } \\
\text { FCF, HCF } \\
\text { HCF, HCF } \\
\text { HCF, HCF } \\
\text { HCF, HCF }\end{array}$ & $\begin{array}{r}0.130 \\
0.106 \\
0.105 \\
-0.136 \\
-0.063 \\
0.073 \\
0.996 \\
-0.305 \\
0.346 \\
0.249 \\
-0.253 \\
-0.286 \\
-0.053 \\
-0.160 \\
-0.048 \\
-0.161 \\
-0.222\end{array}$ \\
\hline
\end{tabular}

- All bending coordinates are weighted by the equilibrium $\mathrm{C}-\mathrm{H}$ bond length. An I matrix element with one subecript is a diszonal element. When two off-diagonal elements are listed to s similar interaction, c.s., $f_{\mathrm{m}}$ and $f_{\mathrm{m}}$ the one without a prime refers to the interaction between iwo coordinates which share a common C-H bond, while the one with - prime refers to the interaction between two coordiantes which do mot share a common bond. 'For $f_{\text {ma }}$ and $f_{\text {wo }}$ they share a C-H hond and - C-F rond, respectively.
Table 13. F-matrix of Liquid Methyl Fluoride (1)

\begin{tabular}{ll}
\hline lement & value, mdyn $A^{-1}$ or mdyn A \\
\hline$f_{T_{x}}, f_{T_{y}}$ & 0.120 \\
$f_{T_{z}}$ & 0.300 \\
$f_{R_{x}}, f_{R_{y}}$ & $8.1527 \times 10^{-2}-\left(6.3040 \times 10^{-4}\right) \Delta+$ \\
$f_{R_{z}}$ & $\left(3.6677 \times 10^{-1}\right) \Delta^{2}$ \\
$f_{D T_{z}}$ & $f_{R_{x}} / 8$ \\
$f_{O T_{z}}$ & $\left(169 \times 10^{-2}+\left(1.0788 \times 10^{-3}\right) \Delta+\right.$ \\
$f_{O T_{z}}$ & $\left(1.6045 \times 10^{-1}+\left(7.1425 \times 10^{-4}\right) \Delta+\right.$ \\
& $-f_{B T_{2}}$
\end{tabular}

- All eloments are in units of mdyn $A^{-1}$ except $f_{R}$ 's, which are in unite of mdyn $A$. $\Delta \equiv T-153$.
Table 15. F-matrix of Liquid Methylene Difluoride en

\begin{tabular}{|c|c|c|c|}
\hline \multicolumn{2}{|c|}{ diagonals } & \multicolumn{2}{|c|}{ ofr-diagonals } \\
\hline Dotation & value, mdyn $A^{-1}$ & notation & value, mdyn $A^{-1}$ \\
\hline $\begin{array}{l}f_{0} \\
f_{0} \\
f_{0} \\
f_{0}\end{array}$ & $\begin{array}{l}4.882 \\
5.959 \\
0.462 \\
0.983 \\
0.630\end{array}$ & $\begin{array}{l}f_{00} \\
f_{D D} \\
f_{\infty 0} \\
f_{\infty 0} \\
f_{\infty} \\
f_{\infty} \\
f_{\infty D}\end{array}$ & $\begin{array}{r}0.131 \\
0.106 \\
0.105 \\
-0.136 \\
-0.063 \\
0.073 \\
0.869\end{array}$ \\
\hline \multirow[t]{2}{*}{ 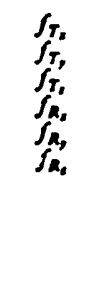 } & $\begin{array}{l}0.300 \\
0.200 \\
0.300 \\
0.200 \\
0.070 \\
0.300\end{array}$ & $\begin{array}{l}S_{\infty} \\
S_{\infty} \\
S_{\infty} \\
S_{\infty} \\
S_{\infty} \\
S_{\infty} \\
S_{\infty} \\
S_{\infty} \\
S_{\infty}\end{array}$ & $\begin{array}{r}-0.314 \\
0.398 \\
0.239 \\
-0.263 \\
-0.283 \\
-0.053 \\
-0.157 \\
-0.055 \\
-0.156\end{array}$ \\
\hline & & $S_{m}$ & -0.217 \\
\hline$n_{11} R_{1}=f_{n_{2}}$ & $f_{n_{1} a_{1}}=-\int_{\text {no }_{2} R_{1}}$ & $\begin{array}{r}3.059 \times 10^{-2} \\
(1.130 \times \\
-4.920 \times 10\end{array}$ & $\begin{array}{l}\left(1.529 \times 10^{-4}\right) \Delta+ \\
\Delta^{2} \\
\left(1.120 \times 10^{-3}\right) \Delta\end{array}$ \\
\hline
\end{tabular}

- All elements are in adya $A^{-1}$, excepe $f_{R}$ and $f_{\text {an }}$ which are in andyn $A$ and adya, nempectively. $\Delta=T-190$. 
Table 16. F-mutrix of Gaseous Fluoroform ${ }^{\text {nt }}$

\begin{tabular}{|c|c|c|c|c|}
\hline \multicolumn{3}{|c|}{ Dingonal. } & \multicolumn{2}{|c|}{ Odf-dingonals } \\
\hline Dencription & Notation & $\begin{array}{c}\text { Value } \\
(m \text { mdyn/A) }\end{array}$ & Notetion & $\begin{array}{c}\text { Value } \\
\text { (mdya/A) }\end{array}$ \\
\hline $\begin{array}{l}\text { C-I stretch } \\
\text { C-F stretcb } \\
\text { LCF bond } \\
\text { FCF bend }\end{array}$ & $\begin{array}{l}f_{0} \\
f_{0} \\
f_{0} \\
f_{0}\end{array}$ & $\begin{array}{l}5.0000 \\
6.0179 \\
0.4913 \\
0.7646\end{array}$ & 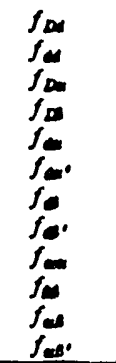 & $\begin{array}{r}1.1720 \\
0.7499 \\
-0.1674 \\
0.1674 \\
0.1943 \\
-0.2657 \\
0.2560 \\
-0.1808 \\
0.0393 \\
-0.0973 \\
-0.1517 \\
-0.2667\end{array}$ \\
\hline
\end{tabular}

The internal coordinate system cons lete of one $\mathrm{CH}$ bond

etretch $D$, three CF bond atretches $d$, three HCF angle bends a, and three FCF angle bends B. (See FLg. 4.) All bending coordinates are welghted by the equllibrlum C-F bond length of 1.332 $\mathcal{A}$. An $\mathbf{z}$ matrix element with one aubscript is a dleconal alement, and one with two subscripts th an off-diagonal alament. When two off-diagonal elements are listed for a simllar interaction, e.g. , $f_{a}$ and $f_{a x}$, the one without a prime reders to the interection between two coordinates whicb share a common C-F bond, whlle the one with a prime refere to the ineraction betweas two coordinate which do not ahare a common C-r bond.
Table 17. P-matrix of Liquid Fluoroform ${ }^{\text {an }}$

\begin{tabular}{|c|c|c|c|c|}
\hline \multicolumn{3}{|c|}{ Diagonale } & \multicolumn{2}{|c|}{ Ot-diagonels } \\
\hline Description & Notation & $\frac{\text { Value }}{(m d y n / A)^{*}}$ & Notatlon & $\begin{array}{c}\text { Value } \\
(m \text { dyn } / \lambda)^{b}\end{array}$ \\
\hline $\begin{array}{l}C-H \text { stretch } \\
C-F \text { otretch } \\
\text { HCF bend } \\
\text { FCF bend }\end{array}$ & $\begin{array}{l}f_{r_{x}} \\
f_{r_{2}} \\
f_{r_{1}} \\
f_{n_{x_{1}}} \\
f_{n_{1}} \\
f_{n_{1}}\end{array}$ & $\begin{array}{l}0.20 \\
0.20 \\
0.10 \\
0.20 \\
0.20 \\
0.05\end{array}$ & 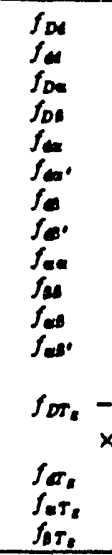 & $\begin{array}{c}1.0400 \\
0.6650 \\
-0.1674 \\
0.1674 \\
0.1943 \\
-0.2657 \\
0.0256 \\
-0.1808 \\
0.0393 \\
-0.0973 \\
-0.1517 \\
-0.2667 \\
\\
73+5.0 \times 10^{-3} \\
-165 \times 1 \\
0.13 \\
0.13 \\
-0.13\end{array}$ \\
\hline
\end{tabular}

For definitions of the internal coordinates and $F$-matrix element notations, see the footnote of Table IV.

'All $\mathbf{F}$-matrix elements are in the unlts of mdyw/ $A$ except $f_{R}$ 's, which are in the unite of mdyn $A$.

From the first-order orthogonal approximation (cf: Section Il of present report), we expect the effects of non-isotopic substituents on isotope effects to be additive to the extent that the force constants are transferrable among different chemical species. Then, $T$ ln $\left(P^{\prime} / P\right)$ per $D-$ for-H substitution would be approximately similar. This is roughly the case between the D/H-VPIE's of methyl fluoride and fluoroform, but it is not so with methylene difluoride (Observation 4). Table 18 provides a quantitative explanation. The methane data were taken from References (136-138). From Table 18, ${ }^{(22)}$ the following points are noted.

a) The magnitudes of the shift in $\delta(Z P E)$ upon condensation, $\Sigma \delta \Delta v_{i}$, for the hydrogen isotope effects is significantly greater than that for the carbon isotope substitutions, as expected.

b) The shifts, $\Sigma \delta \Delta v_{i}(D / H)$ for the fluoromethanes are negative, while that in methane is positive. The negative shifts, which is an anomaly rather than a norm, are due mainly to the large blue shifts in the $\mathrm{C}-\mathrm{H}$ stretching frequencies upon condensation, i.e., $v_{1}$ in $\mathrm{CHF}_{3}, v_{1}$ and $v_{6}$ in $\mathrm{CH}_{2} \mathrm{~F}_{2}$, and $\mathrm{V}_{1}$ and $\mathrm{v}_{4}$ in $\mathrm{CH}_{3} \mathrm{~F}$. The blue-shifts in fluoroform and methyl fluoride have been qualitatively explained on a basis of hydrogen bonding in molecular orbital studies. ${ }^{(16,20)}$ This explains why the D/H-effects in the fluoromethanes are normal and large while the $\mathrm{D} / \mathrm{H}$-effects in methane is inverse (Observation 1). 
Table 18. Compariese of Frequency Shifts in Fluoromethenes (a)

\begin{tabular}{|c|c|c|c|c|c|c|}
\hline \multirow{2}{*}{$\begin{array}{l}\text { Auoroform } \\
165 \mathrm{~K}\end{array}$} & \multicolumn{2}{|c|}{${ }^{12} \mathrm{CHF}$, } & \multicolumn{2}{|c|}{${ }^{12} \mathrm{CDF}_{3}$} & \multicolumn{2}{|c|}{${ }^{14} \mathrm{CHF}_{2}$} \\
\hline & $v_{1}^{\prime}(\mathrm{gas})$ & $\Delta_{1}^{\prime}$ & $\Delta \nu_{1}$ & $\Delta \Delta v_{1}$ & $\Delta \boldsymbol{u}_{1}$ & $\Delta \Delta v_{1}$ \\
\hline $\begin{array}{l}v_{1}\left(A_{1}\right) \\
v_{2}\left(A_{1}\right) \\
v_{1}\left(A_{1}\right) \\
v_{1}(E) \\
v_{3}(E) \\
v_{6}(E) \\
\text { colals }\end{array}$ & $\begin{array}{r}3036.2 \\
1138.9 \\
701.4 \\
1381.0 \\
1151.8 \\
507.7\end{array}$ & $\begin{array}{r}-26.3 \\
20.4 \\
4.6 \\
5.4 \\
-7.9 \\
-0.2 \\
-6.7\end{array}$ & $\begin{array}{r}-18.3 \\
22.6 \\
3.0 \\
-6.1 \\
1.7 \\
0.0 \\
-1.5\end{array}$ & $\begin{array}{r}-8.0 \\
-2.2 \\
1.6 \\
11.5 \\
-9.6 \\
-0.2 \\
-5.2\end{array}$ & $\begin{array}{r}-26.2 \\
18.8 \\
4.3 \\
5.8 \\
-8.1 \\
-0.2 \\
-8.1\end{array}$ & $\begin{array}{r}-0.1 \\
1.6 \\
0.3 \\
-0.4 \\
0.2 \\
0.0 \\
1.4\end{array}$ \\
\hline \multirow{2}{*}{$\begin{array}{l}\text { methylene } \\
\text { dinluoride } \\
\text { at } 190 \mathrm{~K}\end{array}$} & \multicolumn{2}{|c|}{${ }^{12} \mathrm{CH}_{2} \mathrm{~F}_{2}$} & \multicolumn{2}{|c|}{${ }^{12} \mathrm{CD}_{2} \mathrm{~F}_{2}$} & \multicolumn{2}{|c|}{${ }^{18} \mathrm{CH}_{2} \mathrm{~F}_{2}$} \\
\hline & $v_{i}^{\prime}(\mathrm{gas})$ & $\Delta v_{i}^{\prime}$ & $\Delta v_{1}$ & $\Delta \Delta y_{1}$ & $\Delta \nu_{1}$ & $\Delta \Delta v_{1}$ \\
\hline $\begin{array}{l}m_{1}\left(A_{1}\right) \\
m_{2}\left(A_{1}\right) \\
v_{1}\left(A_{1}\right) \\
m_{1}\left(A_{1}\right) \\
m_{3}\left(A_{2}\right) \\
m_{0}\left(B_{1}\right) \\
m_{1}\left(B_{1}\right) \\
m_{0}\left(B_{2}\right) \\
m_{1}\left(B_{2}\right) \\
\text { toculs }\end{array}$ & $\begin{array}{r}2947.0 \\
1507.1 \\
1107.3 \\
532.3 \\
1260.6 \\
3014.2 \\
1183.3 \\
1436.9 \\
1081.2\end{array}$ & $\begin{array}{r}-16.0 \\
-0.1 \\
28.4 \\
1.1 \\
-2.2 \\
-15.8 \\
13.0 \\
-0.4 \\
-7.9 \\
0.1\end{array}$ & $\begin{array}{r}-10.7 \\
15.3 \\
11.5 \\
1.3 \\
1.1 \\
-10.2 \\
15.7 \\
-5.5 \\
-2.8 \\
15.7\end{array}$ & $\begin{array}{r}-5.3 \\
-15.4 \\
16.9 \\
-0.2 \\
-3.3 \\
-5.6 \\
-2.7 \\
5.1 \\
-5.1 \\
-15.6\end{array}$ & $\begin{array}{r}-16.1 \\
-1.3 \\
27.8 \\
1.2 \\
-2.6 \\
-15.9 \\
12.3 \\
-0.2 \\
-7.8 \\
-2.6\end{array}$ & $\begin{array}{r}0.1 \\
1.2 \\
0.6 \\
-0.1 \\
0.4 \\
0.1 \\
0.7 \\
-0.2 \\
-0.1 \\
2.7\end{array}$ \\
\hline \multirow{2}{*}{$\begin{array}{l}\text { methyld } \\
\text { nuoride at } \\
153 \mathrm{~K}\end{array}$} & \multicolumn{2}{|c|}{${ }^{12} \mathrm{CH}, \mathrm{F}$} & \multicolumn{2}{|c|}{${ }^{12} \mathrm{CD}, \mathrm{F}$} & \multicolumn{2}{|c|}{${ }^{13} \mathrm{CH}_{3} \mathrm{~F}$} \\
\hline & $D_{i}^{\prime}(\mathrm{gas})$ & $\Delta v_{i}^{\prime}$ & $\Delta v_{1}$ & $8 \Delta n_{1}$ & $\Delta y_{1}$ & $S \Delta \nu_{1}$ \\
\hline \multirow[t]{2}{*}{$\begin{array}{l}v_{1}\left(A_{1}\right) \\
v_{2}\left(A_{1}\right) \\
v_{1}\left(A_{1}\right) \\
v_{0}(E) \\
v_{3}(E) \\
v_{0}(E) \\
\text { totals }\end{array}$} & $\begin{array}{l}2909.8 \\
1462.6 \\
1056.8 \\
3006.7 \\
1479.3 \\
1183.0\end{array}$ & $\begin{array}{r}-5.3 \\
5.6 \\
61.7 \\
-23.6 \\
-1.0 \\
1.3 \\
15.4\end{array}$ & $\begin{array}{r}3.5 \\
18.8 \\
28.9 \\
-12.8 \\
-3.1 \\
0.9 \\
21.2\end{array}$ & $\begin{array}{r}-8.8 \\
-13.2 \\
32.8 \\
-10.8 \\
2.1 \\
0.4 \\
-5.8\end{array}$ & $\begin{array}{r}-6.0 \\
6.1 \\
61.5 \\
-24.0 \\
-0.7 \\
1.3 \\
14.8\end{array}$ & $\begin{array}{r}0.7 \\
-0.5 \\
0.2 \\
0.4 \\
-0.3 \\
0.0 \\
0.6\end{array}$ \\
\hline & \multicolumn{2}{|c|}{${ }^{12} \mathrm{CH}_{4}$} & \multicolumn{2}{|c|}{${ }^{12} \mathrm{CD}_{4}$} & \multicolumn{2}{|c|}{${ }^{10} \mathrm{CH}_{4}$} \\
\hline methane' & $\sigma_{i}^{\prime}\left(\mathrm{g}^{2 a s}\right)$ & $\Delta v_{1}^{\prime}$ & $\Delta \nu_{1}$ & $\Delta \Delta_{1}$ & $\Delta y_{1}$ & $\Delta \Delta_{y_{1}}$ \\
\hline $\begin{array}{l}v_{1}\left(A_{1}\right) \\
v_{1}(E) \\
v_{1}\left(F_{2}\right) \\
v_{1}\left(F_{2}\right) \\
\text { coldis }\end{array}$ & $\begin{array}{l}3143.7 \\
1574.2 \\
3154.1 \\
1357.4\end{array}$ & $\begin{array}{r}11.5 \\
3.9 \\
13.8 \\
4.9 \\
75.4\end{array}$ & $\begin{array}{r}8.2 \\
2.8 \\
11.5 \\
3.0 \\
57.3\end{array}$ & $\begin{array}{r}3.3 \\
1.1 \\
2.3 \\
1.9 \\
18.1\end{array}$ & $\begin{array}{r}11.5 \\
3.9 \\
13.6 \\
4.9 \\
74.8\end{array}$ & $\begin{array}{l}0.0 \\
0.0 \\
0.2 \\
0.0 \\
0.6\end{array}$ \\
\hline
\end{tabular}

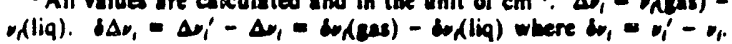

The differences in the $\mathrm{D} / \mathrm{H}$ - and ${ }^{13} \mathrm{C} /{ }^{12} \mathrm{C}$-effects between fluoromethanes and methane are due to the differences in the molecular symmetry and the relative magnitudes of intermolecular forces. The carbon atom in methane does not move at all in all but one of the $F_{2}$ mode. This results in the insignificant contribution to the zero-point energy term. The dominant term for the carbon-VPIE in methane is the one due to the translational lattice modes. In contrast, the peripheral D/H substitutions in methane lead to the large, positive $\delta \triangle(Z P E)$, which is responsible for the inverse D/H-VPIE in methane.

Fluoromethanes being of lower molecular symmetry, the carbon atom actively participates in all normal modes, and many interactions between the internal vibrations and the lattice modes are 
symmetry-allowed. In addition, the intermolecular forces are greater in the liquid fluoromethanes than in methane, as Table 19 implies. This and the blue shifts are responsible for the large, normal D/H-VPIE's

Table 19. Some Properties of Liquid Fluorometheses on

\begin{tabular}{lrccc}
\hline & $\begin{array}{c}\text { normal } \\
\text { bp. } \bullet\end{array}$ & $\begin{array}{c}\Delta H \text { vap } \\
\text { at nbp, } \\
\mathrm{kJ} / \mathrm{mol}\end{array}$ & $\begin{array}{c}\text { dipole } \\
\text { moment } \\
\text { (gas). D }\end{array}$ & $\begin{array}{c}\text { polarizability } \\
\text { (gas), } \mathrm{A}^{\text {( }}\end{array}$ \\
\hline $\mathrm{CH}_{4}$ & -161.5 & 8.4 & 0 & 2.60 \\
$\mathrm{CH}_{3} \mathrm{~F}$ & -78.4 & 17.2 & 1.85 & 2.67 \\
$\mathrm{CH}_{2} \mathrm{~F}_{2}$ & -51.6 & 19.2 & 1.97 & 2.74 \\
$\mathrm{CHF}_{3}$ & -82.0 & 16.7 & 1.65 & 2.81 \\
$\mathrm{CF}_{4}$ & -128.0 & 12.1 & 0 & 2.89 \\
\hline
\end{tabular}

in the fluoromethane. For the carbon VPIE, the inverse effect are due to the redshifts and the fact that the isotopic substitutions are made near the centers of mass and near the principal axes.

An examination of the Figures and Tables of the present section reveals that the rules of thumb discussed in Section IIIB in terms of the theory of $\triangle \delta(Z P E)$ are in agreement with the experimental results.

The unusually large blue-shift in the D/H-efffect of methylene difluoride (Table 18) is responsible for the disparity in the VPIE per D/H substitution (Observation 4). The large shift is primarily due to two reasons. First, $\mathrm{CH}_{2} \mathrm{~F}_{2}$ being of the lowest molecular symmetry, the molecules interact more extensively in the liquid. Secondly, since $\mathrm{CH}_{2} \mathrm{~F}_{2}$ has two hydrogen atoms and two hydrogen acceptor atoms, the highest possible average number of hydrogen bonds per $\mathrm{CH}_{2} \mathrm{~F}_{2}$ molecule is two. In contrast, the highest possible average number of hydrogen bonds per $\mathrm{CHF}_{3}$ or $\mathrm{CH}_{3} \mathrm{~F}$ molecule is one. In $\mathrm{CHF}_{3}$ and $\mathrm{CH}_{3} \mathrm{~F}$ there are inbalance between the donor and aceeptor atoms. These observations and Table 19 are also consistent with the fact that the thermal expansion coefficients of liquid methylene difluoride is the smallest of the three fluoromethanes: at 173K, for instance, $\beta=2.2$ for $\mathrm{CH}_{3} \mathrm{~F}, 1.8$ for $\mathrm{CH}_{2} \mathrm{~F}_{2}$, and 2.4 for $\mathrm{CHF}_{3}$.

All observed $\mathrm{D} / \mathrm{H}$ - and ${ }^{13} \mathrm{C} /{ }^{12} \mathrm{C}$-VPIE's can be explained in terms of molecular symmetry and intermolecular interactions in the liquid. 


\section{IV-B. Vapor Pressure Isotope Effects in Liquid and Solid Ammonia}

We studied hydrogen- and nitrogen-VPIEs in liquid and solid ammonia. ${ }^{(30,31)}$ Both VPIEs are normal at temperatures between $163 \mathrm{~K}$ and $243 \mathrm{~K}$. There have been earlier investigations of the VPIEs of ammonia. ${ }^{(135)}$ Figures 17 and 18 compare our results ${ }^{(31)}$ with the earlier data. The solid lines drawn through our data ( the dots) are the least-squared fit lines in the form,

$$
T \ln \frac{p^{\prime}}{p}=\frac{1}{T}-B
$$

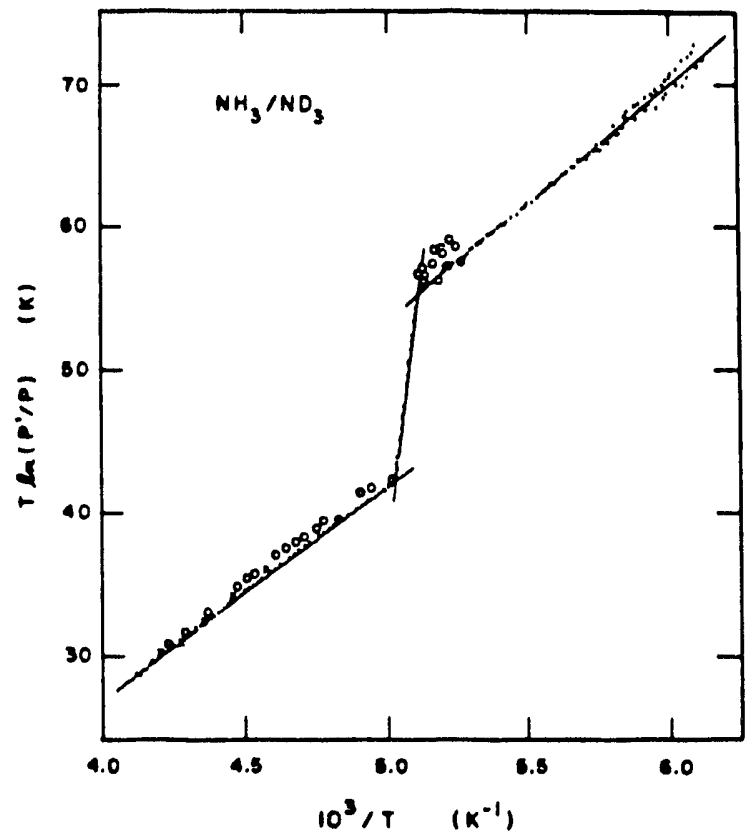

Figure 17. Observed $D / H$ vapor pressure isotope effects in ammonic. Dots; present work, Circles (139); Crosses (140).

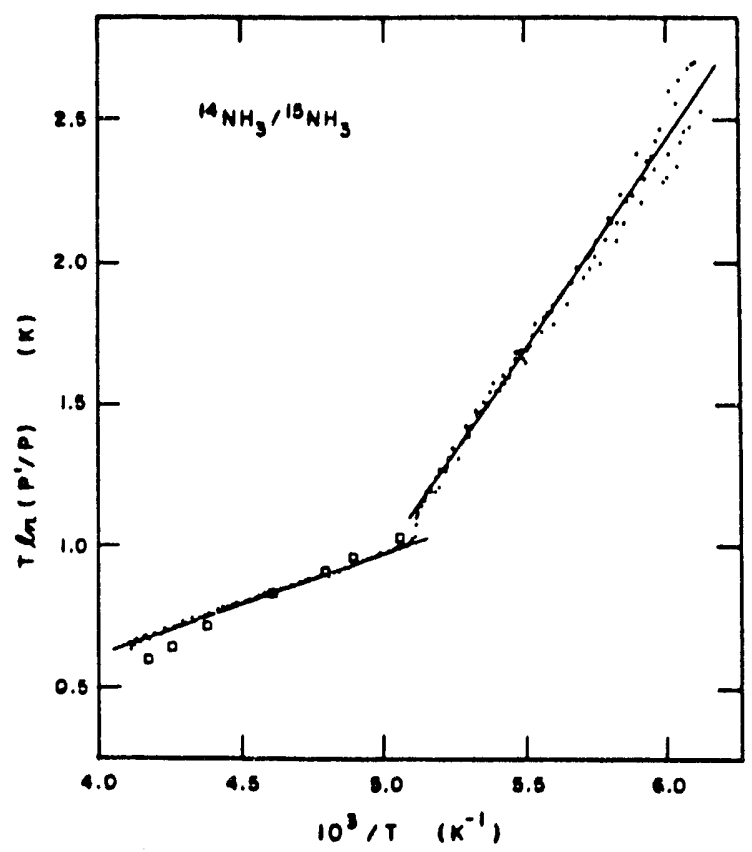

Figure 18. Observed ${ }^{15} \mathrm{~N} /{ }^{14} \mathrm{~N}$ vepor pressure isotope effects in ammonis. Dots; present work. Squares (141).

The parameters A and B are tabulated in Table 20 and may be physically interpreted by in Eqs. (133) and (134). The vapor pressures of the individual isotopic ammonia can be represented by

and

$$
\begin{aligned}
& \ln P(\text { torr })=a-\frac{b}{T}-c T \quad[\text { liquid }] \\
& \ln P(\text { torr })=a-\frac{b}{T} \quad \text { [solid] }
\end{aligned}
$$

where $T$ is in $K$. The least-squares fit parameters are summarized in Table 21. 
Table 20. Leat-equares fits of isotopic vepor presaure mtios to Eq. (135). ${ }^{\text {gu }}$

\begin{tabular}{|c|c|c|c|c|}
\hline $\begin{array}{l}\text { Ioropic } \\
\text { Puir }\end{array}$ & $\begin{array}{l}\text { Temperafure } \\
\text { range (K) }\end{array}$ & $A$ & $\mathbf{B}$ & $\bullet$ \\
\hline iq do/liqd, & $T_{w}<T$ & $\begin{array}{l}15041.2 \\
\pm 129\end{array}$ & $\begin{array}{l}33.1387 \\
\pm 0.1976\end{array}$ & 0.1025 \\
\hline $\mathrm{Had} d_{0} / \mathrm{eOl}_{3}$ & $T_{*}^{\prime}<T<T_{*}$ & $\begin{array}{l}161084 \\
\pm 5414\end{array}$ & $\begin{array}{l}768.112 \\
\pm 27.426\end{array}$ & 0.778 \\
\hline$\omega \mid d_{0} / \operatorname{sol}_{3}$ & $T<T_{*}^{\prime}$ & $\begin{array}{l}16768.7 \\
\pm 152.5\end{array}$ & $\begin{array}{l}29.9744 \\
\pm 0.8487\end{array}$ & 0.4715 \\
\hline iq 14/hiq 15 & $T_{u}<T$ & $\begin{array}{r}364.550 \\
\pm 3.105\end{array}$ & $\begin{array}{l}0.83480 \\
\pm 0.01438\end{array}$ & 0.00795 \\
\hline col 14/2ol 1s & $T<T_{*}^{\prime}$ & $\begin{array}{r}1477.21 \\
\pm 20.90\end{array}$ & $\begin{array}{l}6.40848 \\
\pm 0.11658\end{array}$ & 0.06242 \\
\hline
\end{tabular}

$T^{\prime}$ and $T_{\text {. }}$ are the triple points (cf: Table 22) of the firt- and recondnamed ieotopic apecies, repectively. $\sigma$ is the root mens equares deviation.

Table 21. Vapor presaure equations for individual isotopic ammonias: ${ }^{\text {1) }}$ Eq. (136) for the solid, Eq. (137) for the liquid.

\begin{tabular}{|c|c|c|c|c|c|c|c|}
\hline Species & Phase & $\sigma(\log P)$ & $a$ & $b$ & $c$ & $T(K)$ & $\begin{array}{l}\text { Number } \\
\text { of pis }\end{array}$ \\
\hline $\begin{array}{l}{ }^{4} \mathrm{ND}_{3} \\
{ }^{1 / \mathrm{NH}_{3}}\end{array}$ & $\begin{array}{l}\text { Liq } \\
\text { Sol }\end{array}$ & $\begin{array}{c}0.00091 \\
10.000167 \\
(0.00123 \\
0.00251 \\
10.000669 \\
0.00326 \\
0.00028 \\
0.00289 \\
0.00060 \\
0.00314\end{array}$ & $\begin{array}{r}9.94326 \\
10.07133 \\
9.95028 \\
10.01883 \\
10.00528 \\
9.98379 \\
10.30672 \\
10.23684 \\
9.94265 \\
10.04193\end{array}$ & $\begin{array}{l}1471.633 \\
1485.600 \\
1473.17 \\
1633.785 \\
1630.993 \\
1627.22 \\
1541.893 \\
1700.623 \\
1472.232 \\
1638.775\end{array}$ & $\begin{array}{l}0.0038492 \\
0.0041449 \\
0.0038603 \\
0.0043762 \\
0.0038514\end{array}$ & $\begin{array}{l}196-243 \\
199-242 \\
196-250 \\
163-195 \\
177-195 \\
173-195 \\
199-243 \\
163-198 \\
196-243 \\
163-195\end{array}$ & $\begin{array}{c}94 \\
11\} \\
109 \\
7 \\
67 \\
67 \\
135 \\
69 \\
102\end{array}$ \\
\hline
\end{tabular}

The triple points obtained by this work are compared with previous results in Table 22. For comparison,

Table 22. Triple points of isotopic ammonis ${ }^{\text {a1) }}$

\begin{tabular}{|c|c|c|c|c|}
\hline \multirow{2}{*}{$\begin{array}{l}\text { Isotopic } \\
\text { opecies }\end{array}$} & \multicolumn{2}{|l|}{$T_{w}(K)$} & \multicolumn{2}{|l|}{$P_{-}$(torr) } \\
\hline & Armstrong & This work & Armstrong & This work \\
\hline $\begin{array}{l}{ }^{4} \mathrm{NH}_{3} \\
{ }^{4} \mathrm{ND}_{3} \\
{ } \mathrm{NH}_{3}\end{array}$ & $\begin{array}{l}195.45 \\
199 \\
-\end{array}$ & $\begin{array}{l}195.41 \\
198.96 \\
195.58\end{array}$ & $\begin{array}{l}45.58 \\
48.22 \\
-\end{array}$ & $\begin{array}{l}45.49 \\
48.35 \\
48.83\end{array}$ \\
\hline
\end{tabular}

the triple point for $\mathrm{NH}_{3}$ obtained by

Overstreet and Giauque (143) is $195.41 \mathrm{~K}$

(45.58 torr) after a $0.05 \mathrm{~K}$ temperature scale correction. Also, Armstrong's triple point temperatures were based on the ice point of 273.16K.

It is notable that the difference in the vapor pressures between $\mathrm{NH}_{3}$ and $\mathrm{ND}_{3}$ does not change much at the temperatures

between the triple points of $\mathrm{NH}_{3}$ and $\mathrm{ND}_{3}$, while the magnitudes of the vapor pressures change considerably ( $P$ ' from about 45.5 torr to about 60.4 torn) in the same range. See Figure $19 .{ }^{(31)}$ The difference in the pressures in this range can be represented by

$$
\delta \mathrm{P}(\text { torr })=\mathrm{P}^{\prime}-\mathrm{P}=-(0.0251 \pm 0.0323) \mathrm{T}(\mathrm{K})+(16.388 \pm 6.381)
$$

From Clausius-Clapeyron equation, this means $P^{\prime} \Delta H_{v e p} \cong P \Delta H_{v q p}$ and $P^{\prime} / P \cong \Delta S_{m b} / \Delta S_{v q p}^{\prime}>1$. From the data, we have $\Delta H$ (sublimation) $/ \Delta H^{\prime}$ (vaporization) $=1.286 \pm 0.012$, while the average of $P^{\prime} / P$ is 1.275 . This phenomenon is still being investigated.

The experimental data of $P^{\prime} / P$ was reduced to $f_{e} / f$ using the same method that has been described in this report in relation to the VPIE of fluoromethanes. A normal coordinate analysis of the condensed phase ammonia molecules resulted in the F-matrices which are summarized in Table 23 for the solid and 
Table 24 for the liquid. For the gas phase, we used Ŝpirko's anharmonic function, ${ }^{(14)}$ which well reproduces all known frequencies of the gaseous isootopic ammonia including the doublets resulting from the umbrellatype inversion. The other F-matrices reproduce all known intemal and lattice frequencies of solid ammonia $\left(\mathrm{NH}_{3}\right.$ and $\left.\mathrm{ND}_{3}\right)$. There are no experimental lattice frequency data for

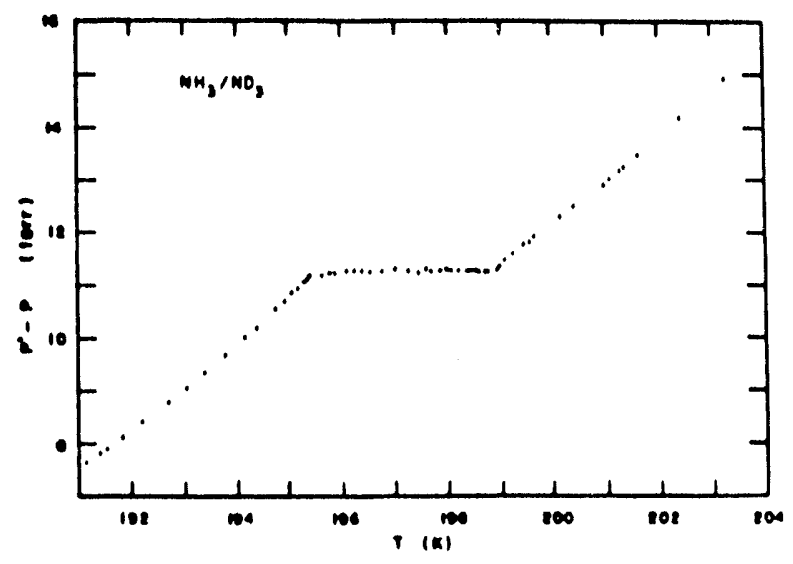

Figure 19. $8 \mathrm{P}=\mathrm{P}\left(\mathrm{NH}_{3}\right)-\mathrm{P}\left(\mathrm{ND}_{3}\right) \mathrm{Vs}$. T near triple points.

${ }^{15} \mathrm{NH}_{3}$. Relative contributions of the

internal and external motions to the VPIE vary with the temperature. Table 25 illustrates the magnitudes of these contributions at typical temperatures.

We derived the following conclusions.

1) Unlike the VPIE in liquid fluoromethanes, the slope of the plots of Figures 17 and 18 are attributable primarily to the translational and rotational motions of the ammonia molecules. The contributions from the internal vibrational motions are very significant but not as temperature-dependent as those in the liquid fluoromethanes.

2) The rotational motions of ammonia molecules in the liquid are strongly hindered. The degree of the hindrance against rotation in the liquid is about the same as in the solid.

3) The rotation of ammonia in the liquid is strongly hindered mainly because of the external forces acting against the motions of hydrogen atom effecting the molecular rotation and not as much because of the forces acting against nitrogen motions.

4) There are significant differences between the liquid and the solid in the forces that resist movements of the nitrogen atom in the directions perpendicular to the molecular figure axis.

5) There are strong interaction between the internal and external modes, espeically in the solid.

These conclusions are in qualitative agreement with the following experimental and theoretical results in the fields other than isotope effects.

a) The $x$-ray studies on the liquid over a wide range of temperatures (199 277K) shows that the close-packed structure (12 nearest neighbors) of the solid persists, more or less, not only through the phase change but also over a range of liquid temperature. 
Table 23. F-matrix of solid ammonis in valence coordinales"

\begin{tabular}{|c|c|c|}
\hline Dmeription & Notation & $\begin{array}{l}\text { Value } \\
\text { (mdya } A^{-1} \text { of mdya } \lambda \text { ) }\end{array}$ \\
\hline 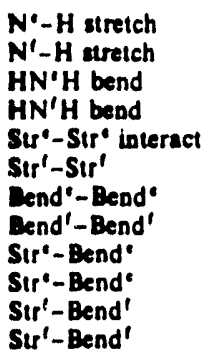 & 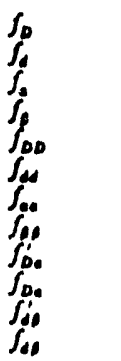 & $\begin{array}{l}5.835 \\
5.834 \\
0.569 \\
0.569 \\
-0.041 \\
-0.011 \\
-0.0040 \\
-0.0041 \\
0.046 \\
-0.0426 \\
0.046 \\
-0.0426\end{array}$ \\
\hline Translation & $\begin{array}{l}f_{i, n}, \\
\text { sf. }^{\prime}\end{array}$ & $\begin{array}{l}0.158-\left(1.8061 \times 10^{-3}\right) \Delta \\
+\left(8.7505 \times 10^{-4}\right) \Delta^{2} \\
0.1154-\left(7.4132 \times 10^{-4}\right) \Delta \\
0.3360-\left(3.4349 \times 10^{-3}\right) \Delta \\
+\left(1.5771 \times 10^{-4}\right) \Delta^{2} \\
0.1012-\left(5.2011 \times 10^{-4}\right) \Delta\end{array}$ \\
\hline Rolation & 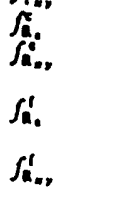 & $\begin{array}{l}0.1558-\left(5.1828 \times 10^{-4}\right) \Delta \\
8.5272 \times 10^{-2} \\
-\left(2.5960 \times 10^{-4}\right) \Delta \\
1.0989 \times 10^{-1} \\
-\left(3.3455 \times 10^{-4}\right) \Delta \\
0.2054-\left(6.6728 \times 10^{-4}\right) \Delta\end{array}$ \\
\hline Exi-Exi & $\begin{array}{l}f_{T_{0}-T_{1}}^{\prime} \\
f_{R_{2}-R_{n}}^{\prime}\end{array}$ & $\begin{array}{l}9.9600 \times 10^{-2} \\
-\left(4.4630 \times 10^{-4}\right) \Delta \\
+\left(7.4074 \times 10^{-6}\right) \Delta^{2} \\
6.5534 \times 10^{-2} \\
-\left(2.5868 \times 10^{-4}\right) \Delta\end{array}$ \\
\hline $\mid n t-E x t$ & 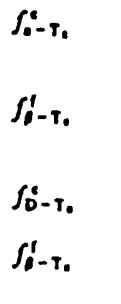 & $\begin{array}{l}-1.000 \times 10^{-1} \\
-\left(4.1183 \times 10^{-3}\right) \Delta \\
-\left(8.4982 \times 10^{-3}\right) \Delta^{2} \\
1.000 \times 10^{-3} \\
+\left(4.0429 \times 10^{-3}\right) \Delta \\
-\left(2.5714 \times 10^{-4}\right) \Delta^{2} \\
\left(5.8181 \times 10^{-3}\right) \Delta \\
+\left(2.6252 \times 10^{-4}\right) \Delta^{2} \\
\left(-1.0315 \times 10^{-2}\right) \Delta \\
-\left(6.8580 \times 10^{-6}\right) \Delta^{2}\end{array}$ \\
\hline
\end{tabular}

- All elements are in units of mdyn $A^{-1}$, except f 's and the interactions among $f$ 's, which are in units of mdyn $X$ $\Delta=T(K)-180$. The superscripts, $c$ and $l_{\text {, denote the cornet }}$ and face molecules, respectively. The primed f(e.g. , fi, refers to an interaction between an NH strecch and an $\mathrm{HNH}$ bend in which the nitrogen atom is the only atom shared by the iwo coordinates, while the unprimed $\int\left(e . q ., \int_{p 0}\right.$ ) refers to the one between the iwo coordinates in which an $\mathrm{N}-\mathrm{H}$ bond is shared. For the external modes, the molecular symmetry axis is the $2-a x i s$. The ootation such is $f_{T}$ means that the element applies to the translation in the molecular $x y$-plane. while f a refers to an interection between the coordinates $T$, and $t$,
Table 24. F-matrix of liquid ammonis in valence coordinales

\begin{tabular}{|c|c|c|}
\hline Dencription & Notation & $\begin{array}{l}\text { Value } A^{-1} \text { or mdyn } A \text { ) } \\
\text { (mdyn } A^{-1} \text { of }\end{array}$ \\
\hline $\begin{array}{l}\mathrm{N}-\mathrm{H} \text { utretch } \\
\mathrm{HNH} \text { bend }\end{array}$ & $\begin{array}{l}f_{f,} \\
f_{p,} \\
f_{p:}\end{array}$ & $\begin{array}{l}6.015 \\
0.529 \\
-0.003 \\
-0.049 \\
-0.0416 \\
0.044 \\
S_{\operatorname{mom}}=0.1421 \\
=-3.4177 \times 10^{-4} \Delta\end{array}$ \\
\hline Rotation & $f_{R}$ & $\begin{aligned} S_{1}= & 0.1040-8.6319 \times 10^{-3} \Delta \\
& +1.8428 \times 10^{-6} \mathrm{~d}^{2}\end{aligned}$ \\
\hline $\begin{array}{l}\text { Internal-Extemal } \\
\text { Interaction }\end{array}$ & fot. $_{\text {pt. }}$ & $\begin{array}{l}+0.0268 \\
+0.0042\end{array}$ \\
\hline
\end{tabular}

All clements are iq units of mdyn $A^{-1}$ except fa's which are in units of mdyn $\lambda .4=T-21$.

Table 25. Comparison of various contributions to $T$ (n $\left(f_{c} / f_{b}\right)$ in isotopic smmonins. All values in $K$.

\begin{tabular}{|c|c|c|c|c|}
\hline & \multicolumn{2}{|c|}{$\begin{array}{l}T \ln \left(5,15_{0}\right) \\
\text { at } 195.41 \mathrm{~K}\end{array}$} & \multicolumn{2}{|c|}{$\begin{array}{l}T \ln \left(S_{1} / S_{1}\right) \\
\text { at } 198.98^{\prime} \mathrm{K}\end{array}$} \\
\hline & $d_{0} / d_{3}$ & $14 / 15$ & $d_{0} / d_{3}$ & $14 / 15$ \\
\hline \multirow{3}{*}{$\begin{array}{l}\text { Translations } \\
\text { Rotations } \\
\text { Internal } \\
\text { vibrations } \\
\text { Non-clasaical } \\
\text { rotations } \\
\text { Total }\end{array}$} & $\begin{array}{r}3.0 \\
84.4\end{array}$ & $\begin{array}{l}6.07 \\
1.48\end{array}$ & $\begin{array}{r}2.6 \\
76.7\end{array}$ & $\begin{array}{l}1.68 \\
0.19\end{array}$ \\
\hline & -31.9 & -6.41 & -37.1 & -0.88 \\
\hline & $\begin{array}{r}1.5 \\
57.0\end{array}$ & $\begin{array}{l}0.0 \\
1.14\end{array}$ & $\begin{array}{r}1.5 \\
43.8\end{array}$ & $\begin{array}{l}0.0 \\
0.99\end{array}$ \\
\hline
\end{tabular}


b) A molecular dynamics study ${ }^{(30)}$ based on a flexible, four-site model of liquid ammonia confirmed the coordination number of 12 , with a slightly more pronounced first neighbor structure at lower temperatures. Other molecular dynamics and Monte Carlo studies also reported the coordination numbers ranging from 12 to 13.

c) According to our molecular dynamics study, ${ }^{(30)}$ there is little directional force on the lonepair side of the nitrogen atom, while the hydrogens behave as if they are tied down by the nitrogen atoms of the neighbor molecules. There are approximately three such nearest neighbor molecules on the hydrogen side..

Thus, the directional preference of the external force on the bydrogen side of an ammonia molecule in the liquid seems to be responsible for the significant rotational contribution in the D/H-VPIE, while the practical absence of directionality of the force on the lone-pair side causes the small rotational contributions in the nitrogen VPIE. 


\section{IV-C. Medium Cluster Model for Vapor Pressure Isotope Effects}

All experimental data on the VPIEs have been explained unexpectedly well by the theories based on the simple cell model of liquid. The model assumes that each liquid molecule is entrapped in an isotropic potential cage, and the surrounding molecules form a structureless environment around the molecule that represents the liquid phase. We developed a new model called medium cluster model 0.8$)$ for liquid, which explicitly takes the intermolecular interactions into account and investigated the reasons why the cell model, which is an obvious oversimplification of liquid structure, apparently works well in explaining the VPIE phenomena.

According to the medium cluster model, a liquid phase is regarded as consisting of a cluster of somewhat regularly arranged molecules. A central molecule is surrounded by a variable number of molecules in the first coordination shell. The method of normal coordinate analysis is used to determine the modes of vibration of the condensed phase cluster taken as a unit, and the RPFR is calculated by using an appropriate number of normal frequencies of the cluster that are attributable to the motions of the central molecule. Clusters of 3, 7,9 and 13 methane molecules in three basic relative orientations of the constituent molecules were examined, and their carbon and hydrogen isotope effects were studied.

Consider a liquid phase consisting of $\mathrm{N}$ molecules to be an assembly of independent clusters with $\mathrm{m}$ molecules each. If the clusters are indistinguishable, the condensed phase partition function can be written as

$$
Q_{c}=\frac{q_{\mathrm{d} / m}^{N / m}}{(N / m) 1},
$$

where $q_{\text {clear }}$ is the complete molecular partition function for the cluster regarded as a macromolecule. Each cluster consists of one molecule at the central position in the aggregate and $m-1$ indistinguishable shell molecules in the first coordination sphere. Using $q_{\text {enerd }}$ and $q_{\text {and }}$ for the molecular partition functions of each type, the cluster partition function may be expressed as

$$
q_{\text {chuster }}=\frac{q^{m-1}}{(m-1) !} q_{\text {contral }} \text {. }
$$

If, in addition, one assumes that the shell and central species are in equilibrium with each other, one has

$$
\frac{q \text { andel }}{q \text { sull }}=\frac{1}{m-1} \text {. }
$$

Substitution of Eqs. (140) and (141) into Eq. (139) and use of the Stirling approximation yield

$$
Q_{c}=\left\{\frac{e^{1 / m}(m-1)(m-1) / m}{[(m-1) !]\left(\frac{N}{m}\right) !}\right\}^{N} .
$$

Then,

$$
\ln \frac{s}{s^{\prime}} f=\frac{1}{N} \ln \left(\frac{Q_{c}}{Q_{c}^{\prime}}\right)_{q m}-\ln \left(\frac{q_{c}}{q_{c}^{\prime}}\right)_{d} .
$$


In accordance with the Born-Oppenheimer approximation, the clusisis size $m$ is isotope-independent and, thus,

$$
\ln \frac{f_{c}}{f_{c}}=\ln \frac{s}{g^{\prime}} f_{\text {contral }}-\ln \frac{s}{s^{\prime}} f_{\text {ges }},
$$

which appears to be identical in its form with the cell-model expression. The counting factors such as those appearing on the right hand side of Eq. (142) have cancelled out between the isotopic molecules. However, the evaluation of $f_{\text {_ar in }}$ in Eq. (144) explicitly takes into account the intermolecular interactions with its closest neighbors.

Since the shell molecules in a cluster are being used to simulate the entire bulk phase, it would be unrealistic to assign the same force constants to the central and shell molecules. The shell force constants must reflect the existence of additional hierarchical coordination spheres and the distribution of energy among central and shell molecules. We have correlated the potential energy surfaces of the central and shell molecules with each other by apportioning the shif in the total electronic kinetic energy upon condensation between the two molecular species of the model through an application of the localized virial theorem. ( 146,107 ) Thus, the F-matrices of the gas (F, ), central (F J), and shell (F ) molecules are related by

$$
F_{s}=F_{z}+\frac{1}{m-1}\left(F_{c}-F_{\varepsilon}\right) \cdot[m>1]
$$

The niodel was applied to the VPIE of ${ }^{13} \mathrm{C},{ }^{14} \mathrm{C}, \mathrm{D}$, and T-substituted methanes using $\mathrm{m}=3,7,9$, and 13. The clusters, $m=7,9$, and 13 , respectively correspond to the simple cubic, body-centered cubic, and face-centered cubic lattice structures. Three equilibrium orientation of methane molecules were considered. They are illustrated in Figure 20 . The results obtained can be summarized as follows.

1) Medium cluster model generally gives better agreement than the cell model with experimental values of the first quantum correction (i.e., A term of Eqs. (132) and (133)) and the ZPE shift term (i.g., B term of Eqs. (132) and (134)) without significantly increasing the number of force constant parameters beyond those needed for the simple cell model.

2) For each equilibrium orientation of Figure 20 , both A- and B-terms exhibit a cyclic behavior as a function of conformational angles, as expected. For the spherical-top isotopic methanes, the cyclic variations are mainly and clearly due to interactions of molecular rotation with bond-angle bending and bond-stretching motions.

3) The effects of changing molecular orientations diminish with increasing cluster size (m), until the variations become negligibly small compared to experimental uncertainties. This is illustrated in Figure 21 for $d_{4} / d_{0}$-effects in the gear geometry and in Figure 22 for ${ }^{13} \mathrm{C} /{ }^{12} \mathrm{C}$ effects in the rocket geometry. In Figures 21 and 22 , the intermolecular direction is the $y$-axis, and $\delta$ is rotational angle of the central molecule around the $y$-axis. 
4) Thus, the formalism of simple cell model can be regarded to implicitly account for intermolecular interactions beyond the nearest neighbor molecules.
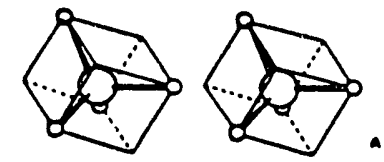

Figure 20. Relative orientation of adjacent methane molecules, $A$ is the rocket geometry, B the antiparallel geometry,
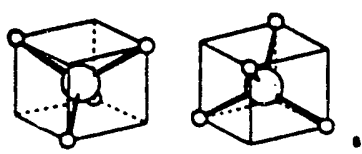
and $C$ the gear geometry."
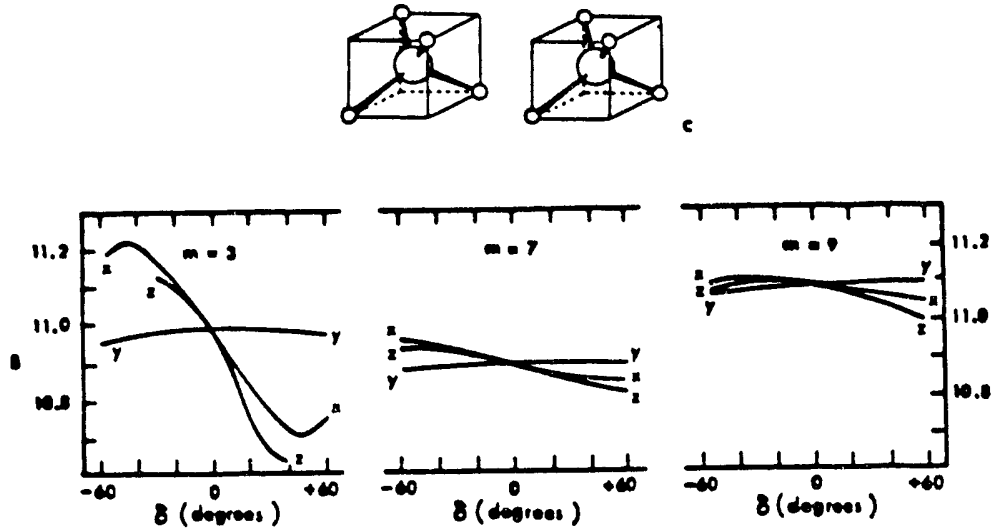

Figure 21. VPIE of $\mathrm{CD}_{4} / \mathrm{CH}_{4}$ for various cluater sizes in the gear geometry as a function of central molecular rotation."
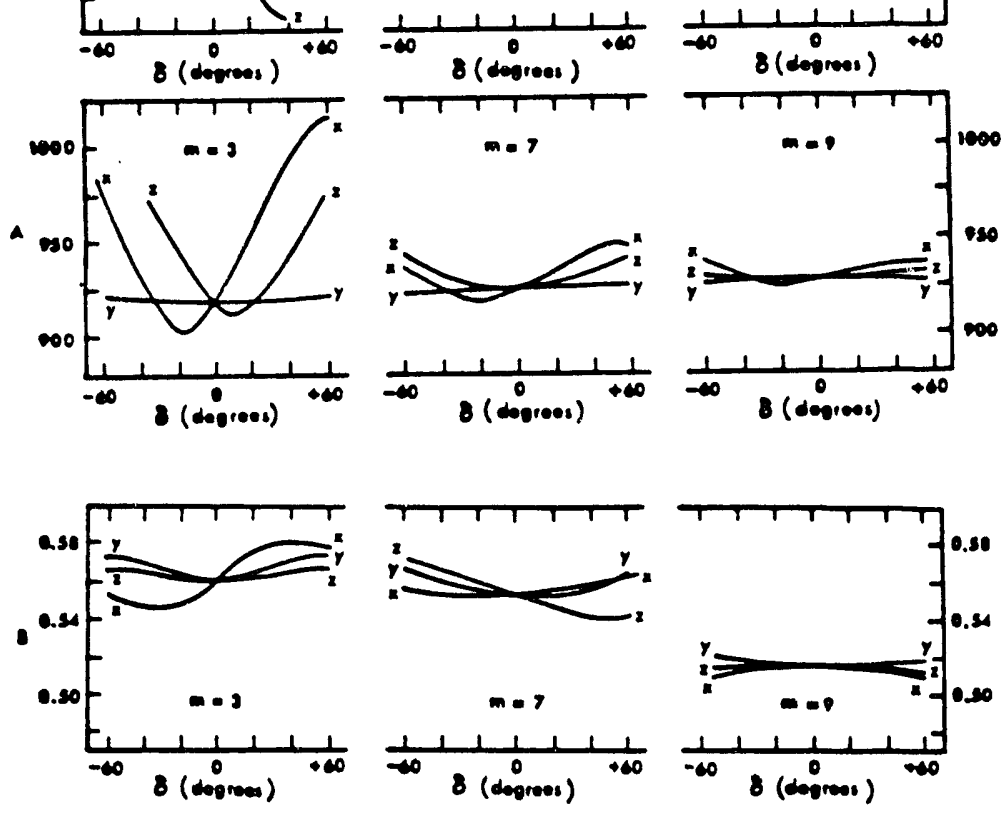

Fiqure 22. VPIE of ${ }^{13} \mathrm{CH}_{4} /{ }^{12} \mathrm{CH}_{4}$ for

various cluster sizell in the rocket geometry
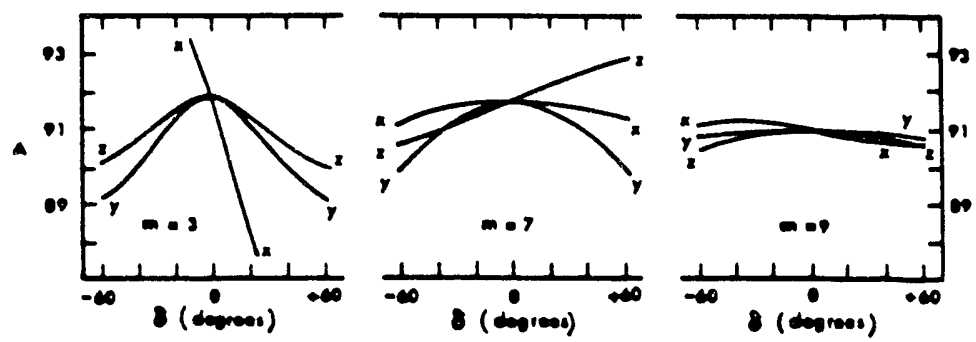

as a function of central molecular rotation") 


\section{Fractionation of Stable Isotopes}

Studies of isotope fractionation will be presented in three sections:

1) Experimental and theoretical studies of ${ }^{13} \mathrm{~N}$ fractionation. ${ }^{(24,148)}$

2) Basic studies for development of hydrophobic catalysts for the exchange reaction of hydrogen isotopes between dihydrogen and water. ${ }^{(162,164.169 .201 .202,203)}$

3) Theoretical studies of transient behaviors of a square cascade for isotope fractionation. ${ }^{(10)}$

\section{V-A. Fractionation of Nitrogen Isotopes}

The cryogenic distillation of nitric oxide and the Nitrox process have been the main processes for producing highly enriched nitrogen-15. The latter utilizes the exchange of nitrogen isotopes between nitric oxide gas and nitric acid, the main exchange reaction being

$$
{ }^{15} \mathrm{NO}(\mathrm{g})+\mathrm{H}^{14} \mathrm{NO}_{3}(\mathrm{aq}) \rightleftharpoons{ }^{14} \mathrm{NO}(\mathrm{g})+\mathrm{H}^{15} \mathrm{NO}_{3}(\mathrm{aq}),
$$

for which the unit stage separation factor $\alpha$ (cf: Eq. (4) on page 2) is 1.055 at $25^{\circ} \mathrm{C}$ and 1 atm pressure when $10 \mathrm{M}$ nitric acid is used. Typical values of $\alpha$ for the NO distillation range from 1.03 at $110.5 \mathrm{~K}$ to 1.007 at $172.8 \mathrm{~K}^{(149)}$

In the Nitrox process, the product-end refluxing is most commonly provided by a chemical reduction using $\mathrm{SO}_{2}$, the main reaction being

$$
2 \mathrm{HNO}_{3}+3 \mathrm{SO}_{2}+2 \mathrm{H}_{2} \mathrm{O} \rightarrow 3 \mathrm{H}_{2} \mathrm{SO}_{4}+2 \mathrm{NO} .
$$

To produce ${ }^{15} \mathrm{~N}$ of a given enrichment, the rate of product withdrawal is limited by the minimum reflux ratio, $R_{\min }$, i.e., the ratio of the refluxed stream flow rate (i.e., nitrix oxide here) to that of the product withdrawal rate. For instance, $R_{\text {m }}$ for the Nitrox process yielding $99.9 \%{ }^{15} \mathrm{~N}$ from the natural abundance of $0.37 \%$ in one square-cascade is about 5,400 . This means that, to produce one mole of ${ }^{15} \mathrm{~N}, 5,400 \mathrm{x}$ $(3 / 2)=8,100$ moles of $\mathrm{SO}_{2}$ will be needed. The cost of $\mathrm{SO}_{2}$ is by far the largest single non-personnel expense for the operation of a Nitrox plant. If this cost could be reduced, the chemical exchange method for ${ }^{15} \mathrm{~N}$ would become more attractive. 
To explore this possibility, we studied (24, 148) a chemical exchange process based on an isotope exchange between a liquid phase, which is primarily a mixture of liquid $\mathrm{N}_{2} \mathrm{O}_{3}$, and a gas phase, which is in phase and chemical equilibria with the liquid phase. Under the experimental conditions that we studied, the liquid is primarily $\mathrm{N}_{2} \mathrm{O}_{3}$ and the gas is primarily $\mathrm{NO}$, so that this system will be referred to as the $\mathrm{NO}_{2} \mathrm{~N}_{3}$ exchange system.

The amount of $\mathrm{SO}_{2}$ that is stoichiometically required for the $\mathrm{NO} / \mathrm{N}_{2} \mathrm{O}_{3}$ system is one-third of that needed for the nitrox system. Furthermore, as shown in this report, it turned out ${ }^{(24)}$ that the HEPT (the height equivalent of theoretical plate) for the $\mathrm{NO} / \mathrm{N}_{2} \mathrm{O}_{3}$ packed-column system is almost an order of magnitude smaller than the HEPT for the NO/ $\mathrm{HNO}_{3}$ operating under similar conditions.

\section{V-A-1. Equilibrium Properties of NO/N $\mathbf{N}_{2} \mathbf{O}_{3}$ System}

First, the phase and chemical equilibrium data in literature on the liquid and gaseous system involving $\mathrm{NO}, \mathrm{NO}_{2}, \mathrm{~N}_{2} \mathrm{O}_{3}$, and $\mathrm{N}_{2} \mathrm{O}$, were systematized to obtain a self-consistent data basis. ${ }^{(148)}$ The only assumption made was that the liquid phase only consists of $\mathrm{N}_{2} \mathrm{O}_{3}$ and $\mathrm{N}_{2} \mathrm{O}_{4}$. This limits the present model to the liquid whose average oxidation number ranges from 3 and 4 . The average atom fraction of +4 nitrogen in the liquid and gas phase are denoted $x$ and $y$, respectively, the mole fractions of $\mathrm{N}_{2} \mathrm{O}_{3}$ and $\mathrm{N}_{2} \mathrm{O}_{4}$ in the liquid are $\mathrm{x}_{3}$ and $\mathrm{x}_{4}$, respectively, and the mole fractions of $\mathrm{NO}, \mathrm{NO}_{2}, \mathrm{~N}_{2} \mathrm{O}_{3}$, and $\mathrm{N}_{2} \mathrm{O}_{4}$ in the gas phase are $y_{1}, y_{2}, y_{3}$, and $y_{4}$, respectively. The calculational model successfully reproduced the following experimental data ${ }^{(148)}$ :

a) Total pressure, $P$, over the liquid mixture as a function of $x$ and $T$,

b) equilibrium constant of

$$
\mathrm{N}_{2} \mathrm{O}_{3}(\mathrm{~g}) \rightleftharpoons \mathrm{NO}(\mathrm{g})+\mathrm{NO}_{2}(\mathrm{~g}) \quad,
$$

as a function of temperature and pressure,

c) equilibrium constant of

$$
\mathrm{N}_{2} \mathrm{O}_{4}(\mathrm{~g}) \rightleftharpoons 2 \mathrm{NO}_{2}(\mathrm{~g}) \quad,
$$

as a function of temperature and pressure, and

d) ratio of partial pressures, $p_{3}$ (for $\mathrm{N}_{2} \mathrm{O}_{3}$ ) to $\mathrm{p}_{4}$ (for $\mathrm{N}_{2} \mathrm{O}_{4}$ ) to the liquid molefractions, $x_{3}$ and $x_{4}$, as a function of temperature.

From the model, it was possible to evaluate all partial pressures and $y$ for a given $x$ and $T$. From this data basis the effective stage spearation factor, $\alpha_{\text {off }}$, was computed as a weighted average of the relevant isotupe exchange reaction; 


$$
\alpha_{\text {eff }}=\frac{\left[\Sigma_{i=1}^{2} x_{i}\left(s / s^{\prime}\right) f_{i}\right]_{\text {liquid }}}{\left[\Sigma_{i=1}^{4} y_{i}\left(s / s^{\prime}\right) f_{i}\right]_{\text {gas }}},
$$

where the sum is taken over all chemical species present in each phase. The effective separation factor as a function of the liquid composition, the total pressure, and temperature are summarized in Figures 23 and 24.
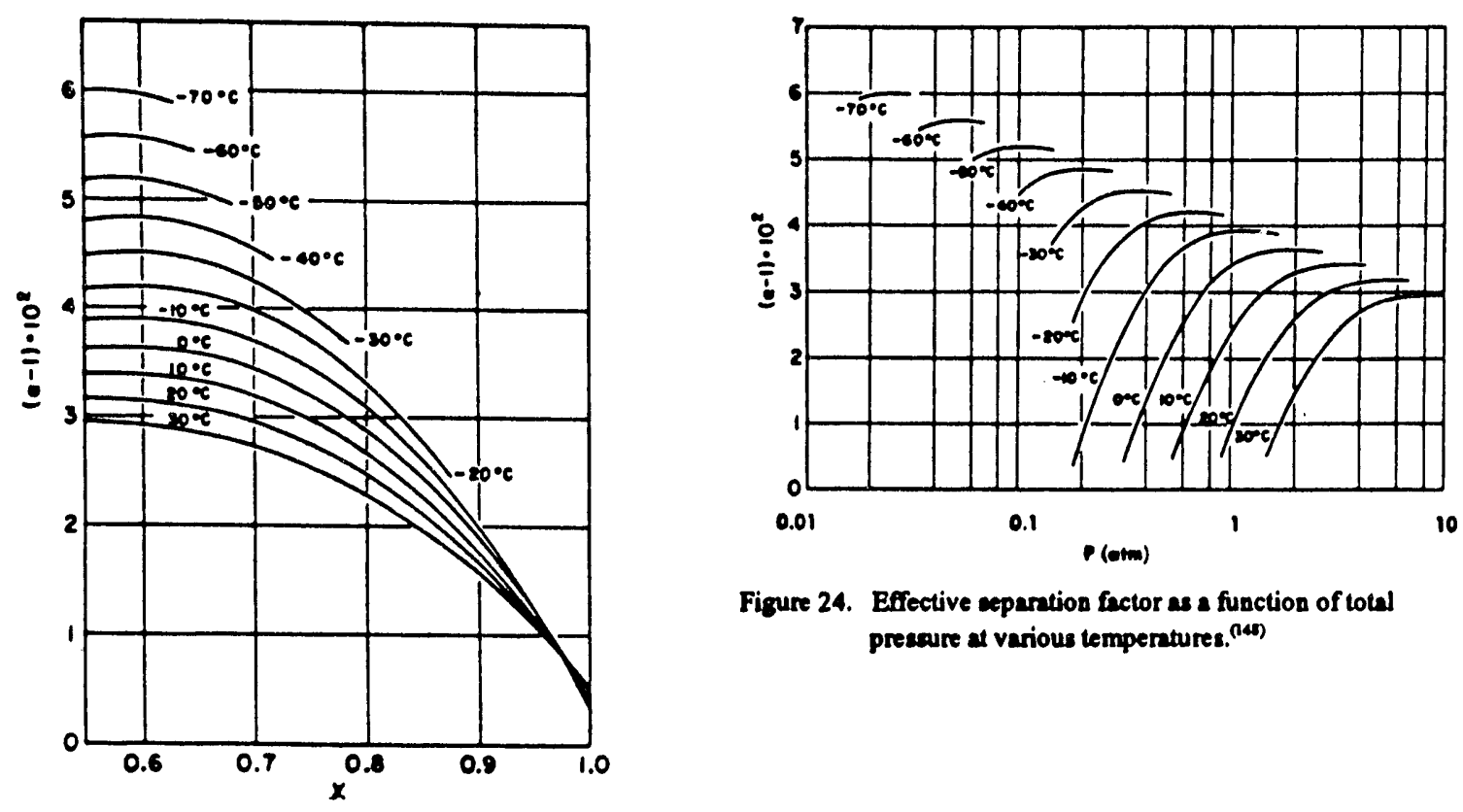

Figure 24. Effective exparation factor as a function of tolal presaure at various temperntures. ${ }^{(a+s)}$

Figure 23. Effective sepanation factor as a function of liquid composition, $x$, various temperatures. ${ }^{\text {nat }}$

The plots go over a maximum at evey temperature. The effective separation factor is increased by the addition of nitric oxide to the system, as long as the added NO decreases the denominator of Eq. (150) for the vapor phase more than the numerator for the liquid phase. As the composition of the vapor phase approaches that of pure NO, a point is reached where further increase in the relative amount of +2 nitrogen in the system affects the gas phase composition less than the composition of the liquid phase; the numerator of Eq. (150) then decreases more rapidly than the denominator, and $\alpha_{\text {eff }}$ thus decreases. These tendencies are in good agreement with experiments. ${ }^{(150)}$

\section{V-A-2. Nitrogen-15 Fractionation Based on the $\mathrm{NO} / \mathrm{N}_{2} \mathrm{O}_{3}$ Exchange System}

The NO/ $\mathrm{N}_{2} \mathrm{O}$ exchange system for ${ }^{15} \mathrm{~N}$ separation was investigated ${ }^{(24)}$ at temperatures between $-9^{\circ} \mathrm{C}$ and $20^{\circ} \mathrm{C}$ and under various pressures up to $4 \mathrm{~atm}$, and $\alpha_{\text {etr }}$ HETP and the liquid and gas phase compositions were determined.

Some typical results are presented in Table 26 and Figure $25{ }^{(24)}$ A comparison with the Nitrox 
Table 26. Comparion of Effects of Operating Parameters on the Size of NO/N $\mathrm{O}_{3}$ Single Exchange Column Syatem: Basis $=18$ of $99 \%$ " $\mathrm{N} /$ day ${ }^{\text {a) }}$

\begin{tabular}{|c|c|c|c|c|c|}
\hline & $\mathbf{~ A A}$ & $m$ & is & $\boldsymbol{\omega}$ & 128 \\
\hline 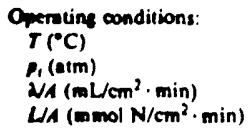 & $\begin{array}{c}-9 \\
1.00 \\
0.35 \\
127\end{array}$ & $\begin{array}{c}+15.5 \\
1.00 \\
0.42 \\
13.5\end{array}$ & $\begin{array}{c}+14.5 \\
3.40 \\
0.37 \\
13.1\end{array}$ & $\begin{array}{c}+15.0 \\
4.08 \\
0.48 \\
17.4\end{array}$ & $\begin{array}{c}+20.0 \\
200 \\
0.42 \\
13.8\end{array}$ \\
\hline $\begin{array}{l}\text { Panmevers obsained: } \\
\text { HETP }\end{array}$ & $\begin{array}{l}1.36 \\
1.02\end{array}$ & $\begin{array}{l}1.006 \\
0.75\end{array}$ & $\begin{array}{l}1.032 \\
1.11\end{array}$ & $\begin{array}{l}1.030 \\
1.07\end{array}$ & 1.000 \\
\hline $\begin{array}{l}\text { Mimimum required parame } \\
U_{\min } \times 10^{-3} \\
U_{\min }(\mathrm{mmol} \mathrm{N} / \mathrm{min}) \\
H_{\min }\left(\mathrm{cm}^{2}\right) \\
V_{\min }\left(\mathrm{cm}^{3}\right)\end{array}$ & $\begin{array}{c}209 \\
7.81 \\
361 \\
295 \\
28.4 \\
8.4 \times 10^{9}\end{array}$ & $\begin{array}{c}1706 \\
45.5 \\
2105 \\
1200 \\
157 \\
20 \times 10^{5}\end{array}$ & $\begin{array}{c}324 \\
8.75 \\
405 \\
360 \\
30.9 \\
1.1 \times 10\end{array}$ & $\begin{array}{c}345 \\
9.31 \\
431 \\
369 \\
24.8 \\
9.2 \times 10^{3}\end{array}$ & $\begin{array}{l}515 \\
13.8 \\
60 \\
525 \\
46.0 \\
24 \times 10\end{array}$ \\
\hline
\end{tabular}

Tuble 27. Comparieon of the NONO Syatem and Nitrox Syatem $\omega$

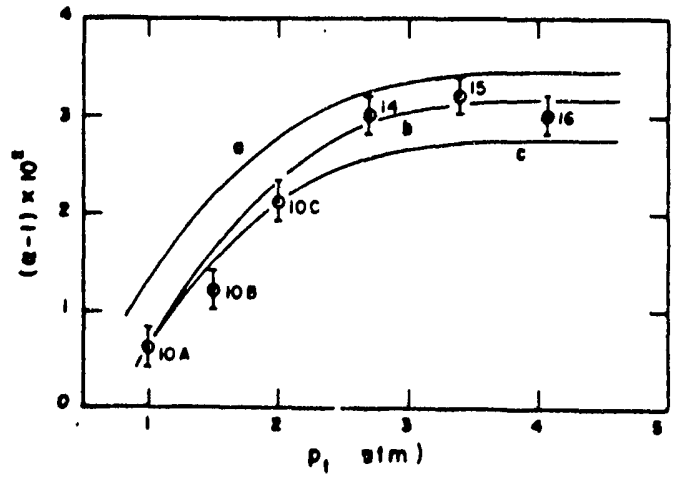

Figure 25. $\alpha_{-\infty}$ as a function of Lolal presaure a $15^{\circ} \mathrm{C}$. The eolid lines are computed from various F-matres.

\begin{tabular}{|c|c|c|c|c|c|c|}
\hline & \multicolumn{3}{|c|}{ NOANO, } & \multicolumn{3}{|c|}{$\mathrm{NON} \mathrm{N}_{1} \mathrm{O}_{1}^{\prime}$} \\
\hline $\begin{array}{l}\text { Operoting perameters: } \\
T\left({ }^{\circ} \mathrm{C}\right) \\
P_{1}(\mathrm{ntm}) \\
N_{A}\left(\mathrm{~mL} \mathrm{~cm}^{2} \cdot \mathrm{min}\right) \\
W_{A}\left(\mathrm{mmol} \mathrm{N} / \mathrm{cm}^{2} \cdot \mathrm{min}\right) \\
\text { HETT } \\
\text { HETP }\end{array}$ & $\begin{array}{l}1.6 \\
17\end{array}$ & $\begin{array}{c}25 \\
1 \\
3.0 \\
32 \\
1.055 \\
4.0\end{array}$ & $\begin{array}{l}5.0 \\
53\end{array}$ & $\begin{array}{c}0 \\
20 \\
0.83\end{array}$ & $\begin{array}{r}14.5 \\
3.5 \\
0.754\end{array}$ & $\stackrel{1.14}{30}$ \\
\hline $\begin{array}{l}\text { Minimum roquired panmet } \\
{ }^{n}\left(L / m_{\min } \times 10^{-3}\right. \\
L_{\min }(\operatorname{mmol} \mathrm{N} / \mathrm{min})\end{array}$ & & $\begin{array}{r}191 \\
4.90 \\
228\end{array}$ & & & $\begin{array}{l}324 \\
8.75 \\
005\end{array}$ & \\
\hline $\begin{array}{l}H_{\min }(\mathrm{cm}) \\
A_{-m}\left(\mathrm{~cm}^{2}\right) \\
V_{\min } \times 10^{-3}\left(\mathrm{~cm}^{3}\right)\end{array}$ & $\begin{array}{l}533 \\
13 \\
7.2\end{array}$ & $\begin{array}{l}364 \\
7.1 \\
3.4\end{array}$ & $\begin{array}{l}1100 \\
4.3 \\
41\end{array}$ & $\begin{array}{l}275 \\
15.6 \\
4.3\end{array}$ & & $\begin{array}{r}194.4 \\
10.4 \\
2.0\end{array}$ \\
\hline
\end{tabular}

- For the symbols, see the footnote of Table 26.

- From Reference (15).

- Based on Run 15.

system is given in Table $27 .^{(24)}$ For instance, at $15^{\circ} \mathrm{C}$, we note the following.

a) At $15^{\circ} \mathrm{C}, \alpha_{\text {orf }}$ increases from 1.006 at $1 \mathrm{~atm}$ to 1.030 at 2.7 atom and levels off thereafter.

b) The HETP correlates well with the linear flow rate of the gas stream, showing a dominant diffusion-control of the overall exchange rate.

c) At $15^{\circ} \mathrm{C}$, the HETP of the $\mathrm{NO} / \mathrm{N}_{2} \mathrm{O}_{3}$ system under $3 \sim 4$ atm can be smaller by a factor of 7 to 10 than that of the Nitrox system running under similar conditions, rendering the $\mathrm{NO}_{2} \mathrm{~N}_{2} \mathrm{O}_{3}$ system a possibility of a significantly smaller plant in spite of its smaller separation factor.

The smaller volume of the $\mathrm{NO} / \mathrm{N}_{2} \mathrm{O}_{3}$ system reduces not only the capital construction cost but also the holdup of the enriched isotope in the plant, thus lowering the plant operating cost. 


\section{V-B. Hydrophobic Catalysts for Hydrogen Isotope Exchange between Dihydrogen and Water}

The exchange reactions of isotopes of hydrogen, H, D, and T, between gaseous dihydrogen and water such as,

$$
\mathrm{HD}(\mathrm{g})+\mathrm{H}_{2} \mathrm{O}(\mathrm{l}) \rightleftharpoons \mathrm{H}_{2}(\mathrm{~g})+\mathrm{HDO}(\mathrm{l}),
$$

are of importance in the nuclear energy technology. The reaction requires a catalyst containing Group VIII metals such as platinum. However, the nucleophilic water molecules tend to preempt the catalyst surfaces, excluding the hydrogen molecules from the active sites and resulting in an extensive retardation of the isotope exchange reactions on the surface.

One solution of the problem is a hydrophobic catalyst such as those developed by Chalk River. However, all heterogeneous catalyst could potentially suffer a loss of catalytic activity due to deterioration of crystallographic surface structure and chemical poisoning.

The goal of the present study is development of a catalyst of new type, which would provide an active control over the surface hydrophobicity and also afford in situ regeneration of the catalytic activity. The concept is as follows. A bed of an electrically conducting porous support material serves as (a) a packing material for a countercurrent, isotope exchange column and (b) a working electrode. The surface of the substrate electrode is chemically modified by electrodeposition of a conducting organic film and a catalyst metal. Basis of this design is two recent developments in electrochemistry.

1) The wettability of electroactive modifiers appears to be adjustable through electrochemical control of the average redox state of the modifier molecule. ${ }^{(151,152)}$ They tend to be hydrophilic in the oxidized states and hydrophobic in the reduced states.

2) Fine aggregates of metals can be elctrochemically imbedded in a polymer film formed on a substrate electrode. ${ }^{(133.136)}$ This was found in an effort to prepare conductive polymers.

These facts suggest that, if an electrode that is modified by an electrochemically active film is used as a support for a catalyst metal such as platinum, one may be able to control its hydrophobic environment for the catalyst surfaces in situ. This is neither electrolysis nor conventional electrocatalysis in that no significant electric current flows through the catalyst bed. Another advantage is the possibility of in situ regeneration of the catalytic activity by periodic potential excursions. It is well known that the catalytic activities of platinized platinum electrodes can be restored by repeated cycling of the potential between cathodic and anodic voltages. (157) The regeneration is at least partially due to crystallographic restructuring of the catalytic sites ${ }^{(158,159)}$ and removal of chemical poisons from the surface. ${ }^{(158,160)}$ 
An account of our investigations for this goal will be presented in five sections; first on a highly controlled measurement of the hydrophobicity of polycrystalline platinum surfaces, second on chemical poisoning and regeneration of platinum surfaces, third on elecrochemical modifications of platinum surfaces by films of poly (p-phenylene), fourth on modifications of platinum surface by other organic films, and fifth on capacitance measurement for the effective surface area of the edge plane of pyrolytic graphite.

Since none of these results have been published, we will report on these results in more detail than usual. The manuscripts in preparation for publication will bear reference numbers $2 x x$ and be tabulated under "Manuscripts in Preparation" on page xvii.

\section{V-B-1. Hydrophobicity of Polycrystalline Platinum Surfaces}

In order to evaluate the hydrophobicity of an electrochemically modified platinum surface, it is first necessary to know the hydrophobicity of a clean platinum surface to serve as a reference point. The literature cites widely scattered values of contact angle formed between water and "clean" platinum surface, ranging from $0^{\circ}$ (e.g, complete wetting) to an upper limit of about $61^{\circ}$. In the literature, the values differeing from $0^{\circ}$ had been assumed to be due mainly to presence of organic contaminants. Several investigators developed elaborate procedures to reduce and eliminate even the grease or oil originating in the atmosphere which come in contact during preparation of the solid surface. Many others developed several methods for preparation of a "reproducible and clean" platinum surfaces. The methods include mechanical abrasion, heat treatments, electropolishing, and uses of $30 \%$ hydrogen peroxide, aqua regia, hot and concentrated nitric acid, and hot dichromate-sulfuric acid. But, the discrepancies had still persisted.

As a result of our investigation reported below, we now believe that the literature values raising from $55^{\circ}$ to $62^{\circ}$ represent clean, oxide-free platinum surfaces, while the surfaces that give the very small contact angles are, if not completely, very well covered with the oxides and hydroxyl species of platinum.

\section{Preparation of Ultra-Pure Water}

The most widely accepted ultra-high purity water for electrochemical works is prepared by Conway's pyrocatalytic distillation of water. ${ }^{(161)}$ The method involves a slow distillation through a rhodium-based catalyst bed heated to $750^{\circ} \mathrm{C}$ in an all-quartz apparatus. We prepared the water by the Conway method and by a new, less time-consuming, distillation procedure involving acidic permanganate solution. ${ }^{(162,201)}$ Figure 26 is a cyclic voltammogram of polycrystalline platinum surface in $0.5 \mathrm{M}$ sulfuric acid prepared from the APD (acid permanganate distillation) water and $18 \mathrm{M} \Omega \cdot \mathrm{cm}$ sulfuric acid purchased from 
AESAR. The platinum is $99.9985 \%$ Puratronic from Johnson Matthey, preconditioned by a newly developed procedure 162,201 ) The voltammogram shows all prominent anodic and cathodic peaks of a well-formulated surface of platinum. The slight shoulder on Trace " $\mathrm{b}$ " in the oxygen region near $1.0 \mathrm{~V}$ corresponds to $1 \times 10^{-4}$ gram-equivalents per liter of impurity. A similar voltammogram obtained in sulfuric acid made from the PCD (pyrocatalytically distilled) water contains three times as much impurity in this region. The APD water passed our purity test involving potentiostating at $-0.28 \mathrm{~V}_{\mathrm{SCE}}$, which is more stringent than Conway's test.

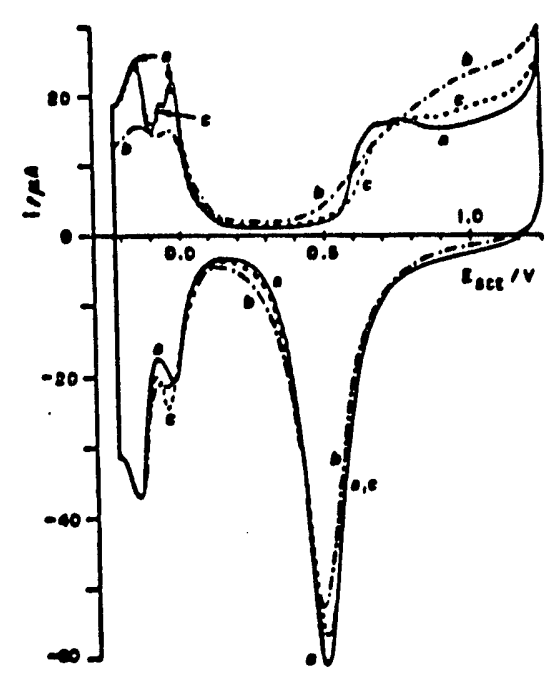

Figure 26. Cyclic voltammogram of polycrystalline platinum in $0.5 \mathrm{M} \mathrm{H}_{2} \mathrm{SO}_{4}$ prepared from APE water and AESAR aulfuric acid: Sweep rate $=100 \mathrm{mV} / \mathrm{s}$. (a) freshly prepared and conditioned $\mathrm{Ph}$, (b) after potentiostating at $-0.28 \mathrm{~V}$ with nitrogen-atirring for 2.5 hrs, (c) after two potential cycles at $100 \mathrm{mV} / \mathrm{s}$ between $-0.225 \mathrm{~V}$ and $1.25 \mathrm{~V}$.

The APE water is much less tedious to prepare than the PCD water, and the apparatus for APD water is much less expensive than that required for the PCD process. We used the PCD water for all of our present investigation.

\section{Hydrophobicity of Platinum}

Figure 27 correlates the contact angle and the EOC (electrode open circuit) potential with the potential at which the platinum electrode was potentiostated (for 15 minutes) prior to the measurements. A $1 / 4^{n}$ rod of 99.99\% Pt was polished in a specially made mount using a series of garnet and diamond powers in APD water and dried in an oil-free, dry nitrogen stream before potentiostating. The Figure shows that both the wettability

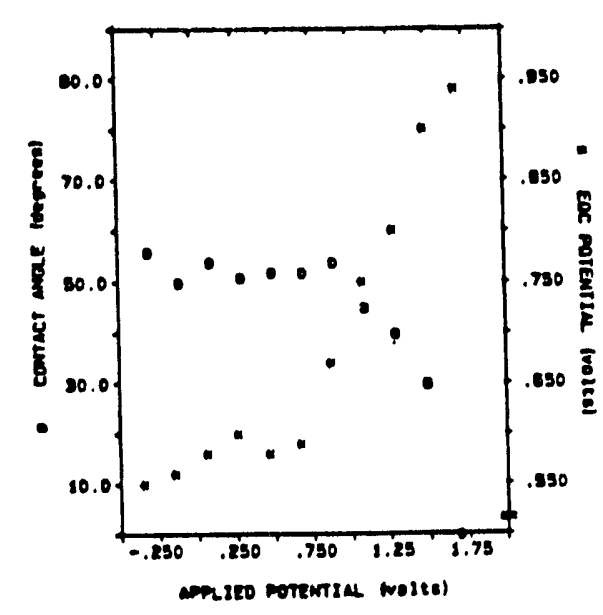
and oxidation state of $P t$ surface remain relatively constant until an applied potential

Figure 27. Contact angle and EOC vs. applied potential. 
of about $0.9 \mathrm{~V}_{\mathrm{scD}}$ is reached. It is above this potential that the surface "oxides" are known to begin to accumulate. As the degree of surface oxidation increases, as indicated by the rise in the electrode open circuit potential, the wettability also increases. After being potentiostated at $+1.7 \mathrm{~V}$ or above, the surface shows a completely wetting.

\section{V-B-2. Chemical Poisoning and Regeneration of Platinum Surfaces}

Reactivation of a heterogeneous catalyst in a process equipment usually involves a shutdown of the equipment and reformation of the catalyst bed. This is an especially time-consuming and costly procedure for an isotope separation equipment because of the long startup period (cf: Section V-C in this report). The cost of shutdown and regeneration of the catalyst bed could be avoided, if the catalytic activity could be regenerated through manipulations of the electrochemical potential associated with the catalyst bed. As far as we are aware, there had been no systematic study of removal of chemical poisons from platinum surfaces.

We carried out such a study ${ }^{(162,201)}$ on the polycrystalline platinum electrodes in sulfuric acid prepared using the AESAR sulfuric acid and the APD water. A platinum working electrode was poisoned in a controlled manner. For each of the catalyst poisons, $\mathrm{Ag}(\mathrm{I}), \mathrm{Cu}$ (II), $\mathrm{Cd}(\mathrm{II}), \mathrm{Pb}(\mathrm{II}), \mathrm{Cr}(\mathrm{III}), \mathrm{Mn}(\mathrm{II})$, $\mathrm{Fe}(\mathrm{II}), \mathrm{Co}(\mathrm{II}), \mathrm{Ni}(\mathrm{II}), \mathrm{Al}(\mathrm{III})$, and bisulfite, which could be present in the industrial process water, the electrode was first potentiostated at $-0.25 \mathrm{~V}_{\mathrm{sCE}}$ for 15 minutes in a $\mathrm{N}_{2}$-stirred, $1 \times 10^{-6} \mathrm{M}$ solution. Then, a series of triangular potential waves between $-0.24 \mathrm{~V}_{\mathrm{SCE}}$ and $+1.25 \mathrm{~V}_{\mathrm{SCE}}$ was imposed, while the electrode was still in the same poison-bearing solution. All solutions were sulfate solution except for $\mathrm{Pb}(\mathrm{II})$,which was a perchlorate solution. The cleaning scans were interspersed with an analytical CV scan. An example of the results is shown in Figure 28.

Figure 28. Recovery of polycrystalline

Pt from Ag-poiconing
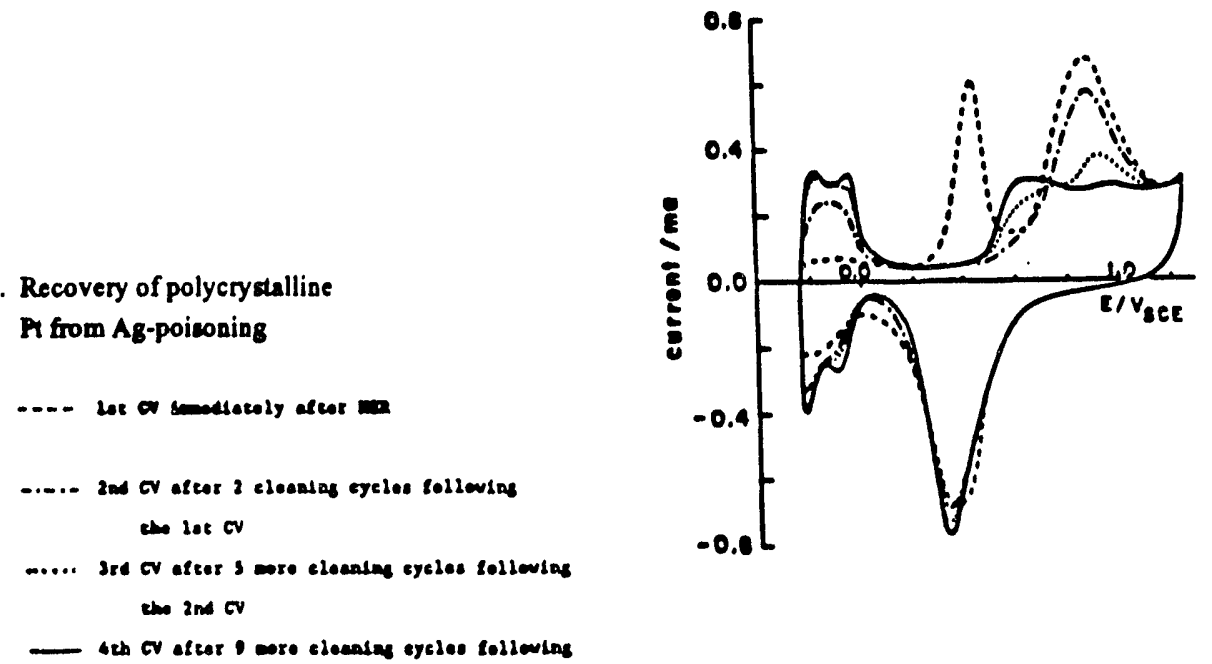

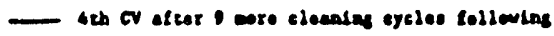

and ard 
In general, the cathodic deposits can be removed und the catalytic activity regenerated by several linear potential scans between these potential even when the solution is quiescent. The reactivation is achieved in one cleaning scan, if the solution is well-stirred.

\section{V-B-3. Electrochemical Modincation of Pt Surface by Poly(p-Phenylene) Film}

The chemical modifier suitable for the present purpose has to satisfy the following requirements:

1) Electrochemical polymerization: The ability for the monomors to polymerize electrochemically allows for a close control over the polymerization process and a uniformity of the modifier film.

2) Wide potential range: The potential range for the electrochemical redox reactions of the modifier film should be sufficiently wide to accommodate various potential excursions, e.g., -0.24 to $+1.25 \mathrm{~V}_{\mathrm{sce}}$ in aqueous solutions.

3) Stability: The film should possess chemical, electrochemical, and mechanical stabilities in various aqueous solution.

4) High hydrophobicity: Contact angles between the surface and water or dilute aqueous solutions of more than $90^{\circ}$ are preferred. In comparison, poly(tetrafluoroethylene) films give a contact angle of aoubt $112^{\circ}$. In addition, a strong dependence of the hydrophobicity on applied potential would make its use more flexible.

5) High electrical conductivity: Under ideal conditions, a high conductivity of the reduced form of the modifier would not be needed for a steady state operation of the isotope exchange column. In order to implement an in situ catalyst regeneration, however, it would be necessary that the film has some degree of electrical conductivity in its reduced form.

We found one such chemical modifier in poly (p-phenylene). ${ }^{(162,202)}$ Preparation and characterization of the film on platinum surface will be summarized below.

\section{Number of Electrons Involved per Monomer in the Electrochemical Polymerization}

The electropolymerization of p-terphenyl has been found to proceed via an intermediate dihydrooligomer dication radicals,

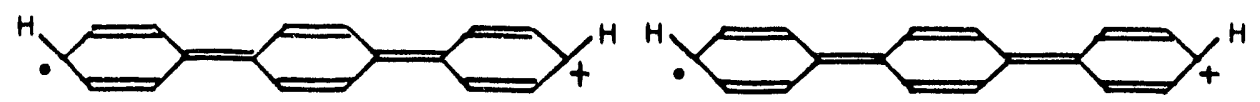


which lose two protons to form a neutral oligomer.

The oxidation of p-terphenyl in this mechanism involves two electrons per monomer. However, the electropolymerization requires more than two eiectrons per monomer to accommodate incorporation (i.e., intercalation) of supporting electrolyte anions (i.e., counter-ions). The overall number of electrons per monomers, $n$, was found by Delahay's method ${ }^{(163)}$ of plotting $\log \left[\left(\tau^{1 / 2}-t^{1 / 2}\right) / t^{1 / 2}\right]$ vs. potential, using a chronopotentiographic data. Here, $\tau$ is the transition time, and the slope of a straight line of the plot is equal to $-\mathrm{nF} / 2.303 \mathrm{RT}$. This method can be applied to every plateau of the chronopotentiogram, and every plateau has a corresponding peak in a cyclic voltammogram. The method was applied to a chrono-

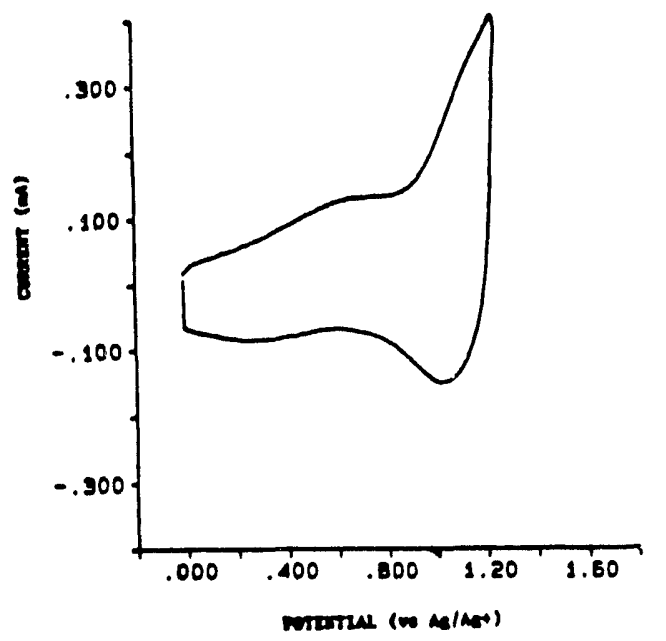

Figure 29. Cyclic voltummogram of poly(p-phenylene) Sweep rate $-1.00 \mathrm{~V} / \mathrm{s}$. The polymer film was grown on a Pt disk in a colution of $2 \mathrm{mM}$ p-terphenyl with $0.1 \mathrm{M}$ TEATFB over a period of 2 seconds at a potential of $+1.40 \mathrm{~V}_{\text {A }} \mathrm{AB}_{\mathrm{B}}$.

potentiographic plateau corresponding to the intercalation shoulder near the anodic switching potential of the cyclic voltammogram such as the one shown in Figure 29. This yields $n_{\text {imered }}=0.2 \pm 0.04$ when the counter ion is $\mathrm{BF}_{4}$, making the total $\mathrm{n}$ about 2.2 in tetraethylammonium tetrafluoroborate (TEATFB) as supporting electrolyte.

\section{Nature of the CV-Peaks}

For the cathodic peak at $1.1 \mathrm{~V}$ in Figure 29, a plot of the peak current, $i_{p}$, as a function of the square root of the CV sweep rate, $v^{1 / 2}$, yielded a parabolic plot representable by

$$
i_{p}(\mu A)=103.8 v-93.6 v^{1 / 2} \text {, }
$$

where $v$ is in seconds. From this, we conclude that the cathodic peak around 1.1V has two equally contributing components; the one which is proportional to $v$ is due to reduction of a surface-immobilized species, which we believe is the polymer, and the other which is propertional to $v^{1 / 2}$ due to deintercalation of a mobile species, which we think is tetrafluoroborate ion. 
The peak current for the $+0.4 \mathrm{~V}$ reduction wave is proportional to the sweep rate, indicating that this wave is due to reduction of an immobilized species, which is contrary to a general believe that this is due to reduction of triphenylenic moieties present in the film. ${ }^{(16)}$ We think this peak represents reduction of the polymer, that is, the reduction of a cationic form of the polymer to a neutral form.

Using Anson's method ${ }^{(163)}$ for determination of $\mathrm{n}$ for a surface-bound species, we obtained $n=1.1 \pm$ 0.3 for the reduction wave at $+0.4 \mathrm{~V}$. We also found that the charge consumed by this reduction wave is almost independent of the duration of polymerization time, while that for the wave at $1.1 \mathrm{~V}$ increases with the polymerization time. Thus, the peak at $0.4 \mathrm{~V}$ only depends on the surface area of platinum. From this, we obtained the surface coverage to be $2.5 \times 10^{14}$ monomer units per $\mathrm{cm}^{2}$ of surface area.

We conclude that

a) the reduction peak at $1.1 \mathrm{~V}$ is half due to the reduction of the polymer and half due to the deintercalcation of $\mathrm{BF}_{4}$,

b) the reduction peak at $0.4 \mathrm{~V}$ is solely due to the reduction of the polymer, and

c) for the peak at $0.4 \mathrm{~V}, \mathrm{n}=1.1 \pm 0.3$, and the charge consumed depends only on the surface area, which fact can be used to determine the surface coverage and the film thickness.

\section{Effects of Supporting Electrolvte}

The three supporting electrolytes shown in Table 28 have sufficiently high solubility in acetonitrile and high stability against oxidation within our potential range of interest, i.e., $0.0 \mathrm{~V}$ to $+1.4 \mathrm{~V}_{\text {Av/Ast. }}$.

Table 28. Properties of Accetable Supporting Electrolytes

\begin{tabular}{|c|c|c|c|c|}
\hline $\begin{array}{l}\text { Supporting } \\
\text { Electrolyte }\end{array}$ & $\begin{array}{l}\text { Reduction (n)) } \\
\text { potential } \\
V\left(\mathbf{A g} / \mathbf{A g}^{*}\right)\end{array}$ & $\begin{array}{l}\text { Oxidation" } \\
\text { polential } \\
\left.V\left(\mathbf{A g} / \mathbf{A}_{\mathbf{B}}\right)^{+}\right)\end{array}$ & Solubility & $\begin{array}{c}\text { Estimuted (k) } \\
\text { size } \\
\left(A^{3}\right)\end{array}$ \\
\hline TEA TFB $^{(n)}$ & -2.0 & $>+2.0$ & v.s. & 52 \\
\hline TEAP(0) & -2.0 & $>+1.8$ & v.s. & 62 \\
\hline TEA-TRF ${ }^{(n)}$ & -2.0 & $>+1.6$ & v.s. & 57 \\
\hline
\end{tabular}

- Potential measured using $1 / 4^{\prime \prime}$ diameter platinum disk electrode.

- Loweat potential attempted.

- Estimated using covalent bond nadii and without solvation.

- Tetrethylammonium tetrafluorobonte

- Tetreethylammonium perchlonte

'Tetnethylammonium triflate: $\left(\mathrm{Et}_{4} \mathrm{~N}\right)^{+}\left(\mathrm{CF}_{3} \mathrm{SO}_{3}\right)^{\text {' }}$

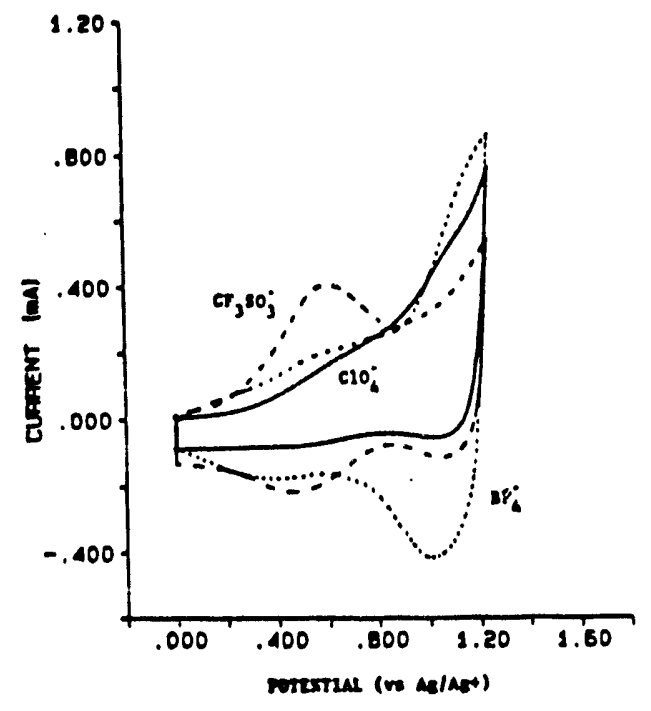

Figure 30. Cyclic voltammogram of poly(p-phenylene) film formed in different mupporting electrolytes: Sweep rate $1.0 \mathrm{~V} / \mathrm{s}$. The CVs were taken in elutions containing the same apporting electrolyte as the polymerization elutions. The films were grown over a period of $10 \mathrm{~s}$. 
The polymerization was performed in each electrolyte. The polymerization current in TEATFB is significantly lower than those in the other two. In Figure 30 we compare the CVs of the polymer films taken in solutions containing the same supporting electrolyte as one in which they were grown.

The following conclusions were obtained.

a) The differences in the shape of the oxidation/reduction couples, their positions, and their intensities are due to differences of stabilities of the films in the counter-ions and to differences in the effects of anion size on intercalation/deintercalation processes.

b) Rousseau et al. ${ }^{(166)}$ observed that porphyrin $\pi$ dications are stable in the presence of $\mathrm{ClO}_{4}$; but react with $\mathrm{BF}_{4}$. They suggest that tetrafluoroborate ion can react with electrochemically generated cations and radical cations. This could be the reason for our observation of the lower polymerization current. An instability of tetrafluoroborate ion could lead to side reactions between the radical cations and anion during the polymerization and result in fewer intercalation sites. On the other hand, the higher intercalation peaks (cf: Figure 30) in the TEATFB solution than in the others is due to the smaller size of $\mathrm{BF}_{4}$ :

c) The polymerization in the perchlorate solution produced a conductive film, which is contrary to other studies. ${ }^{(167)}$.

\section{Stability of Poly ( 0 -Phenylene) Films}

Another criterion for a desirable chemical modifier is a mechanical, chemical and electrochemical stability. All poly (p-phenylene) films that we prepared passed the following stability tests.

a) Wrighton's scotch tape peel test: The color of the film, which ranges from bright blue-green for thin films to bright golden to brown for thicker films, do not change by the test. The CVs do not change by the test.

b) Potential cycling in $0.05 \mathrm{M}$ sulfuric acid between $-0.24 \mathrm{~V}_{\mathrm{scE}}$ and $+1.25 \mathrm{~V}_{\mathrm{sCE}}$ at a rate of 1V/s for 15 hours: The CVs taken before and after the tests in an aqueous solution and in an acetonitrile solution are presented in Figures 31 and 32, respectively.

Figure 31 shows the great enhancement of the features of catalytically active sites of platinum by the potential-cycling, indicating the beneficial restructuring of the exposed platinum surfaces that coexist with the poly(p-phenylene) film. 


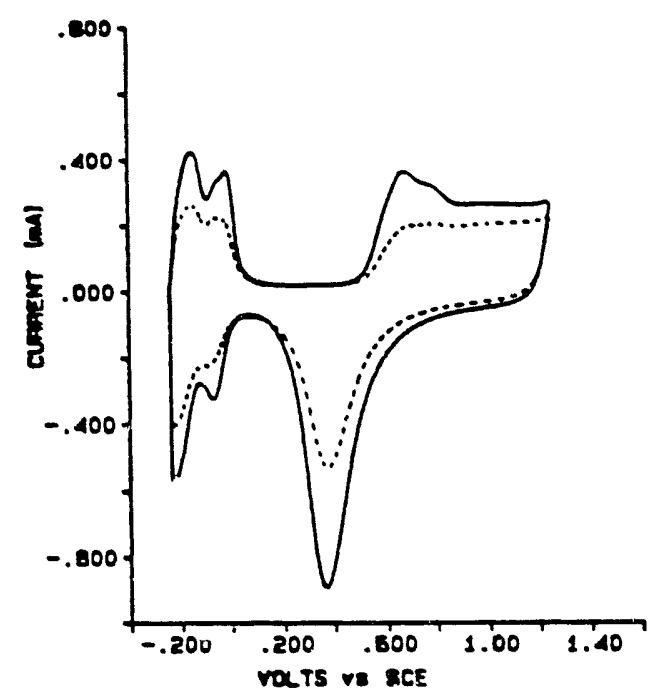

Figure 31. Cyclic voltammogram of platioum wire electrode costed with poly (p-phenylene) in $0.05 \mathrm{M}$ sulfuric acid: Sweep rale $=1.0 \mathrm{~V} / \mathrm{s}$, potential range $=-0.24$ to $+1.25 \mathrm{~V}_{20}$. (a) -.. before cycling, (b) after $15 \mathrm{hr}$ of cycling. The polymer film was grown in acetonitrile solution of $2 \mathrm{mM}$ p-terphenyl and $0.1 \mathrm{M}$ TEA TFB a $+1.30 \mathrm{~V}\left(\mathrm{Ag} / \mathrm{Ag}_{\mathrm{g}}+\right)$ for 20 seconds.

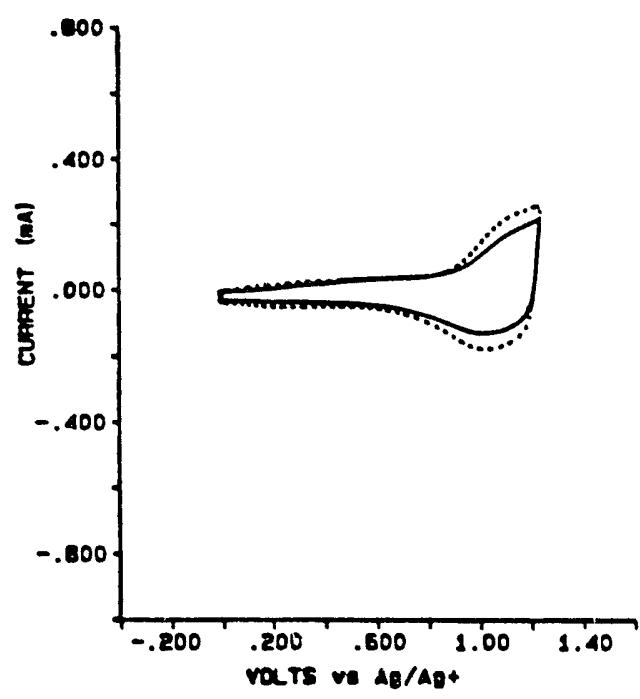

Figure 32. Cyclic voltaminogram of platinum wire electrode coated with poly (p-phenylene) is $0.1 \mathrm{M}$ TEATFB: Sweep rate $=1.0 \mathrm{~V} / \mathrm{s}$, potential nuge $=0.00$ to $+1.25 \mathrm{~V}$ (a) ... before the cycling in alfuric acid, (b) - after the treatment (cf: footnote for Figure 31). The polymer film was prepared in the ame way as in Figure 31.

Figure 32 shows an exceptionally good retention of poly (p-phenylene) characteristics after the very extended potential-excursion period of 15 hours. This is far superior than any other chemical modifiers that have been reported in literature for any purpose.

\section{Hydrophobicity of Poly (o-Phenylene) Films}

The contact angles between APD water and poly (p-phenylene) film on platinum measured in a water vapor-saturated nitrogen atomsphere are summarized in Table 29 . The choice of supporting electrolyte has a definite effect on the wettability of the film. The films produced in TF ATFB become increasingly more hydrophobic as the average thickness increases and reaches a value of $112^{\circ}$ at $1 \mu \mathrm{m}$, which is the same contact angle as poly(tetrafluoroethylene). In contrast, the Iilm grown in TEAP is hydrophilic.

In this connection, we also note that, while the deintercalation peak near $1.1 \mathrm{~V}$ in the CV of the film grown in TEATFB (cf: Figure 29) increases with the increasing film thickness, the similar cathodic peak in the CV of the film grown in the perchlorate environment (not shown) is almost independent of the film 
Table 29. Contact Angles of Poly(p-phenylene) on Pt with water

\begin{tabular}{|c|c|c|c|c|}
\hline \multirow[b]{2}{*}{$\begin{array}{c}\text { Polymerization } \\
\text { time } \\
\text { (eecond) }\end{array}$} & \multicolumn{2}{|c|}{ Tetrnfluomborite } & \multicolumn{2}{|c|}{ Perchlonte } \\
\hline & $\begin{array}{l}\text { Film } \\
\text { thickness } \\
\text { ( } \mu \mathrm{m})\end{array}$ & $\begin{array}{l}\text { Contact } \\
\text { angle } \\
\text { (degrees) }\end{array}$ & $\begin{array}{l}\text { Film } \\
\text { thickness } \\
(\mu \mathrm{m})\end{array}$ & $\begin{array}{l}\text { Contact } \\
\text { angle } \\
\text { (degrees) }\end{array}$ \\
\hline 0 & - & 57 & - & 57 \\
\hline 2 & 0.13 & 80 & 0.11 & 77 \\
\hline 4 & 0.21 & 89 & 0.18 & 54 \\
\hline 6 & 0.28 & 100 & 0.24 & $\$ 2$ \\
\hline 8 & 0.36 & 102 & 0.28 & 50 \\
\hline 10 & 0.41 & 107 & 0.33 & 48 \\
\hline 20 & 0.65 & 112 & - & - \\
\hline 30 & 0.91 & 114 & - & - \\
\hline 40 & 1.33 & 117 & - & - \\
\hline so & 1.68 & 115 & - & - \\
\hline
\end{tabular}

\footnotetext{
- Film thickness was calculated using the experimentally determined covernge of $2.5 \times 10^{14}$ units $/ \mathrm{cm}^{2}$ and $\mathrm{n}=2.2$ for the polymerization reaction in the TEATFB colution.
}

thickness. This is because, perchloroate ions being larger in size than tetrafluoroborate ions, they are trapped in the polymer cage as the polmyer grows and thus do not participate in the charge transport as actively as $\mathrm{BF}_{4}$ :

\section{Conclusion}

We conclude that, among those we have studied, the best chemical modifier for our stated purpose is poly (p-phenylene) film grown in acetonitrile solution containing tetraethylammonium tetrafluoroborate as supporting electrolyte. It is durable and withstands an excessive potential cycling in neutral to acid aqueous media. It possesses a hydrophobicity that is at least as high as "Teflon." Although the polymer itself does not seem to conduct electric charges through the conjugated double-bond chain, the film is sufficiently porous to allow the transport of $\mathrm{BF}_{4}$ ions to the extent that the potential cycling yields the beneficial restructuring of the platinum surfaces. 


\section{V-B-4. Electrochemical Modification of Pt Surfaces by Other Organic Films}

We have also studied the following other possible surface modifiers: ${ }^{(168)}$

(a) poly (2,6-dimethylphenol)

(b) poly $(2,3,4,5,6$-pentafluorostyrene)

(c) tetrafluoro- 1,4-benzoquinone

(d) poly (2, 6-difluorophenol)

The most interesting of these is (a). Polymoerization on a platinum disk electrode was performed by linearly scanning the electrode potential from $-0.5 \mathrm{~V}$ to $1.0 \mathrm{~V}$ ( vs. SCE/MeOH) in methanol solution containing $0.1 \mathrm{M}$ 2,6-dimethylphenol and $0.2 \mathrm{M} \mathrm{NaOH}$. The film of the PPO (polyphenylene oxide) looks blue on the platinum surface. The polymerization current goes over two maxima as the imposed potential was increased linearly as a function of time, indicating a preadsorption mechanism involving lower oligomers. The stability of the PPO film against a potential-cycling in $0.05 \mathrm{M} \mathrm{H}_{2} \mathrm{SO}_{4}$ as determined by cyclic voltammographic and FTIR measurements is satisfactory, although the stability of poly (p-phenylene) films is far superior.

Poly-(2,3,4,5,6-pentafluorostyrene), polyPFS, can be electrochemically prepared by potentiostating a platinum electrode in a $5 \mathrm{mM}$ PFS + 0.2 M TEATFB in acetonitrile, but the polymer does not stay on the platinum surface well. The quinones and hydroquinones do not electrochemically polymerize. Poly(2,6-difluorophenol), poly-DFP, adheres to the platinum surface well and is fairly stable against potential excursions.

\section{V-B-5. Capacitance Measurement for Determination of Effective Surface Area of the Edge Plane of Pyrolvtic Graphite}

Forms of pyrolytic graphite such as reticulated vitreous carbon (RVC) are potential candidate for an acceptable substrate material for the catalyst bed envisioned in the present study. A catalyst, such as platinum, and a chemical modifier, such as poly(p-phenylene) would be codeposited on the substrate, which could also act as a column packing in an isotope exchange column. For further studies we would have to have a means of determination of the effective surface area of such a substrate. In order to provide a basis for such determination, we have investigated ${ }^{(160,203)}$ the electric capacitance measurement methods for the area of the edge plane of the pyrolytic graphite.

Several techniques exist for the measurement of effective area of solid surfaces. Determination of effective area of the edge plane of pyrolytic graphite is especially challenging, because the microfissure openings usually exist on the surface.

The method for the surface area of an electrode based on the electrical capacitance measurement has an important advantage over other common methods such as those using the surface adsorption of 
gaseous molecules in that the capacitance measurement in a solution is non-destructive and tends to yield results which are more representative of the environment in which the electrode will be eventually used. The concept of capacitance measurement is not new, however, a satisfactory procedure has not been established. Here is a report on a method which we believe is promising.

Some explanations of the basics are in order. Suppose first that a conductor electrode is dipped in a solution which contains nothing but an electrochemically inactive electrolyte in a sufficiently high concentration so that the resistance across the solution in the cell is negligible and the capacitance charging is instantaneous. Further suppose that the electrode surface is perfectly planar. Then, the capacitance is

$$
\frac{1}{C}=\frac{1}{C_{H}}+\frac{1}{C_{d}},
$$

where $C_{H}$ and $C_{d}$ are the capacitances of the Helmholtz plane and the diffusion double layer, respectively. Suppose further that a triangular voltage wave is imposed on the electrode; the voltage starts at a cathodic value, $V_{i}$, say, $V_{i}<0$ relative to a reference electrode, linearly increases through $V=0$ until it reaches an anodic switching potential $V_{1}=-V_{i}$, and then linearly decreases through $V=0$ until it gets back to $V_{i}$.

For this idealized electrochemical cell, the output current $i(t)$ looks like Figure 33a, and the capacitance could be calculated from $C=i_{c} / v$, where $i_{c}$ is indicated in Figure $33 a$ and $v$ is the voltage scan rate in volts/sec. If a faradaic current, $i_{p}$ is present, but if the input voltage excursion is not so excessive as to assure that the faradaic current is nowhere near the limiting current, then $i(t)$ would look like Figure $33 \mathrm{~b}$, and the capacitance can be obtained as $C=\left(i_{1}+i_{2}\right) /(2 v)$, where $i_{1}\left(=i_{c}+i_{f}\right)$ and $i_{2}\left(=i_{c}-i_{f}\right)$ are indicated in Figure 33b. In the Cases (a) and (b) a graphical interpretation would directly yield the capacitance. If, in Case (a), the resistive load through the electrolyte solution is finite, the charging current would follow the exponential form,

$$
i(t)=v C\left(1-e^{-t / R C}\right),
$$

where $v$ is again the slope of the voltage scan.

On the other hand, if the electrode surface has sufficiently complicated structure such as the fissures on the real graphite's edge plane, the charging current should be regarded as consisting of two or more parallel capacitors, each of which may be charged at a different rate. It is reminded that only parallel capacitors, and not the capacitors connected in series, can be charged at different rates. Let us call the electrode surface capacitances that charge at indepenent rates distributed capacitance. Figures $33 \mathrm{c}$ and 33d respectively depict such a distributed capacitance without and with a faradaic current.

The charging current plot that we obtained for the edge planes of pyrolytic graphite in acetonitrile solutions of tetraethylammonium tetrafluoroborate (TEATFB) is of Type (d). Previous works on the cases of distributed capacitance have either addressed themselves with complex modelling ${ }^{(170)}$ or used 

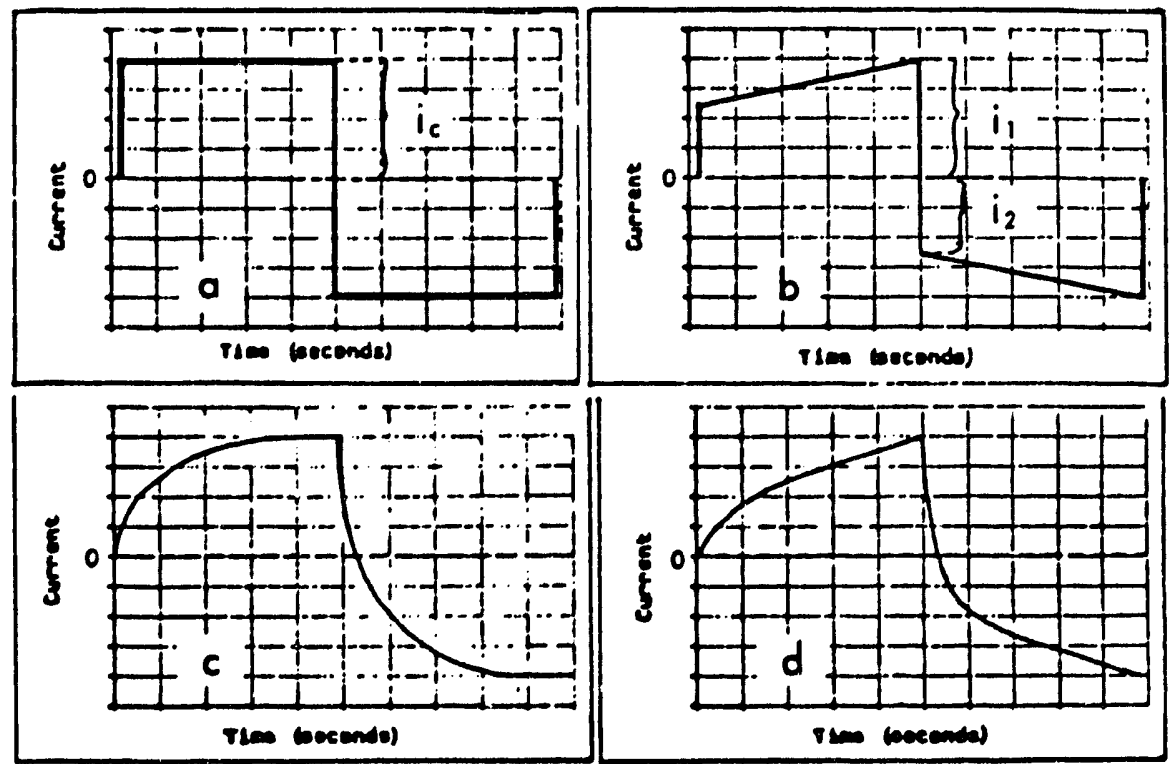

Figure 33. Charging/Discharging Curves: (a) Curreat resposec of an ideal capacitor without a fandaic current. (b) Curreat response is presence of a faredaic curreat. (c) Current response in presence of a distributed capacitance and in absense of a faradaic current. (d) Curreat response in presence of a diatributed capacitance and - faradaic current.

extremely artificial graphical methods. ${ }^{(17)}$ For instance, Figure 34 illustrates one of the graphical methods by Soffer ${ }^{(171)}$, in which the charging/discharging currents are plotted against the applied voltage. Using Soffer's terminology, the "fast charging" ends at Point a where the curve is supposed to suddenly start to deviate from a "vertical line." The vertical line is supposed to represent the fast charging. The region between Points $a$ and $b$ is due to "slow charging." The difference in the current between Points a and $c$ is taken to be equal to twice $i_{f}$, and that between Points $b$ and $c$ is taken to be equal to twice $\left(i_{b}+i_{f}\right)$,

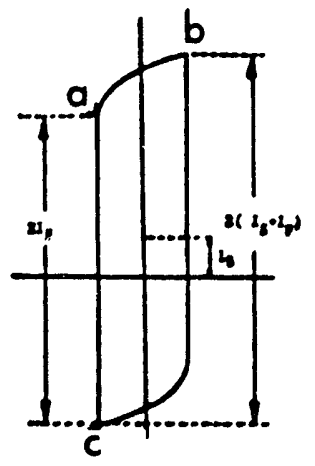

Figure 34. Soffer's graphical method: The vertical axis is current, and the borizontal axis is potential. 
where $i_{f}$ and $i$ are the currents due to the fast and slow charging, respectively. The method suffers from the difficulty and subjectiveness of determination of Point a in Figure 34.

We have fitted the experimental charging curve of the Type $d$ (Figure 33d) to a functional form,

$$
i(t)=v C_{1}\left(1-e^{-t / R_{1} C_{1}}\right)+v C_{2}\left(1-e^{-t / R_{2} C_{2}}\right)+a,
$$

where, again, $v$ is the voltage sweep rate, and $a$ is a constant representing the background current. The results are summarized below.

a) All charging/discharging current data on the edge plane of our pyrolytic graphite can be fitted satisfactorily using only two exponential terms. An example is shown in Figure 35. The saw-tooth trace is the raw experimental data, the saw teeth being due to those superimposed on the input potential ramp produced by a software. The data was deconvoluted to the form of Eq. (155). A single set of parameters fit both the charging and discharging currents equally well, indicating a reversible nature of the transport processes.

b) Inclusion of a faradaic current on the right-hand side of Eq. (155), i.e., a term proportional to the sweep rute, $v$, and time, does not improve the goodness of the fit. This is as expected under our experimental conditions.

c) Figure 36 summarizes the fast and slow charging capacitances of many specimens of vacuum-heat-treated pyrolytic graphite as a function of constant potential superimposed on the analytic potential ramp. The geometric area is $0.0792 \mathrm{~cm}^{2}$, and all capacitance measurements were conducted in 0.1 M TEATFB in acetonitrile. Each point in Figure 26 represents a measurement, in each of which an input triangular wave with an amplitude of 5 $\mathrm{mV}$ and sweep rate of $1 \mathrm{~Hz}$ was applied. The average values of the capacitance correspond to $280 \pm 30 \mu \mathrm{f} / \mathrm{cm}^{2}$ for the fast-charging component and $90 \pm 30 \mu \mathrm{f} / \mathrm{cm}^{2}$ for the slowcharging component. If the thicknesses of the electric double layers of both components are alike, this result would mean that about one quarter of the surface areas of the edge-plane of our specimens of pyrolytic graphite are due to the intra-structure.

d) The vacuum heat treatment of graphite tends to decrease the capacitance, which appear to be due to a decrease in the surface oxygen functionalities. 
e) We have also measured the capacitance as a function of potential scan rate. While the fast capacitance is practically independent of the scan rates in the range between $1 \mathrm{~Hz}$ to $10 \mathrm{~Hz}$, the slow capacitance roughly dropped two-folds as the scan rate was increased from $1 \mathrm{~Hz}$ to $5 \mathrm{~Hz}$ and ten-folds as $v$ was increased from $1 \mathrm{~Hz}$ to $10 \mathrm{~Hz}$. This observation is consistent with a notion that the slow process is due to a charging of an internal structure such as the microfissures underneath the edge plane surface.

Figure 35. Two-component fit of charging/ diecharging currents of edge plane of pyrolytic graphite in $0.1 \mathrm{M}$ TEA TFB in aceto. nitrile: $S$ = slow compnent, $F$ = fest compnent

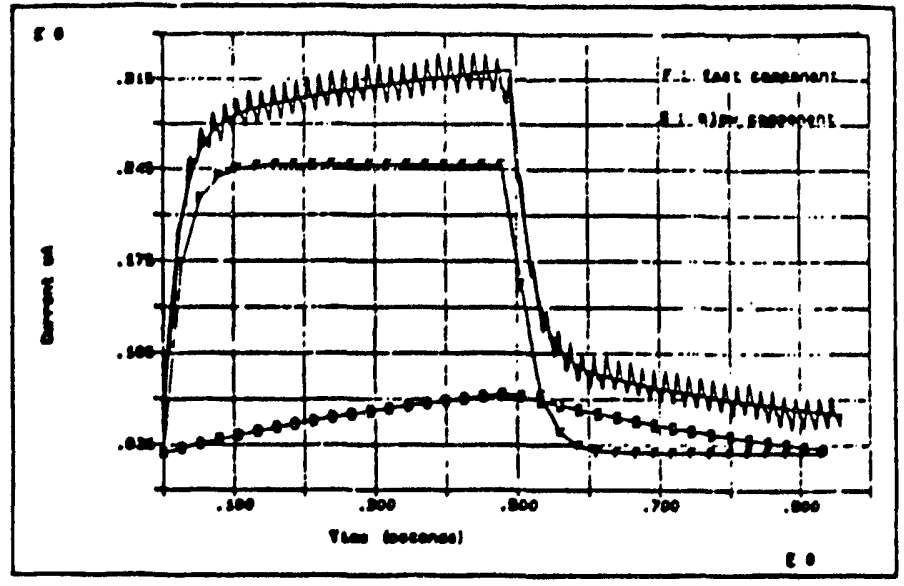

Figure 36. Capacitances of edge plane of pyrolytic graphite as a function of applied potential: Geometric area $=0.0792 \mathrm{~cm}^{2}$. wpporting electrolyte $=0.1 \mathrm{M}$ TEATFB in acetonitrile. (a) fad-charging component. (b) slow-charging component.

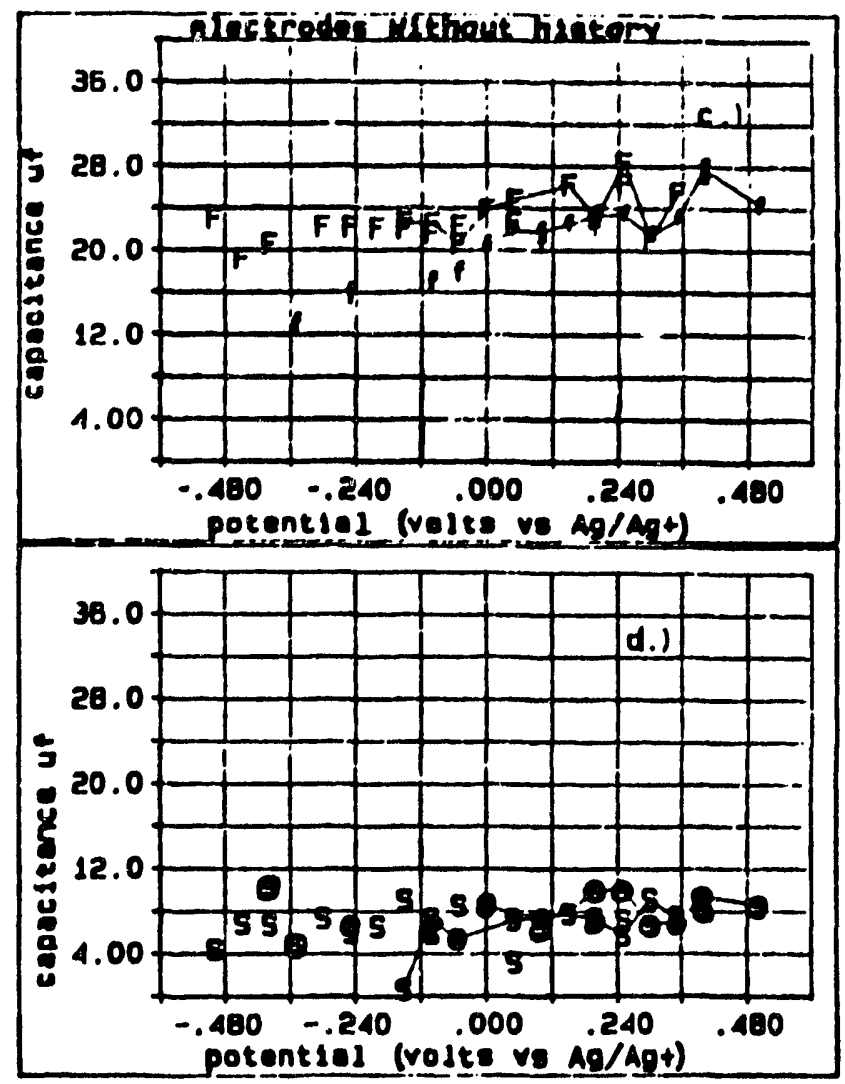




\section{Conclusion}

a) The capacitance measurement is reproducible to within $10 \%$.

b) Since any capacitors connected in series must be charged all at the same rate, the two exponential terms means that there are at least two, significant, parallel charging pathways on the edge- plane surface of the pyrolytic graphite. There are significant internal surface area detectable by the capacitance measurement technique that we have developed. 


\section{V-C. Transient Behavior of Square Cascade of Isotope Separation Stages}

The transient behavior of a square cascasde is important for an efficient operation of isotope separation plants, especially because the stage separation factors $(\alpha)$ of isotope separation processes are close to unity, i.e., $|\varepsilon| \equiv|\alpha-1| \ll 1$. The behavior was theoretically analyzed by Cohen. ${ }^{(172)}$ The theory is useful in a number of ways, for instance, in analyzing the behavior of a plant during a cold start-up period, and in determination of $\alpha$ from laboratory scale experiments. We refined Cohen's theory ${ }^{(10)}$ and pointed out ${ }^{(9)}$ one of its possible consequences regarding the processes based on inverse isotope effects.

Consider the material balance of the isotope that one wants to enrich between the product stage and the s-th stage in a square cascade of separation stages. The consideration leads to the following partial differential equation: ${ }^{(170)}$

$$
\lambda \frac{\partial N}{\partial t}=\frac{\partial^{2} N}{\partial t^{2}}-\varepsilon \frac{\partial}{\partial s}[\Psi N+N(1-N)],
$$

where $\mathrm{N}=\mathrm{N}(\mathrm{s}, \mathrm{t})$ is the average atom fraction of the target isotope in the input streams for the s-th stage at time $t$ after the start-up time of the cascade. The stage numbers are counted starting at the feed point $(s=0)$ toward the product end $(s=n)$. Also, $\lambda=2 h$, where $h$ is the holdup per stage per unit flow, or the average process time per stage, and $\Psi=2 \mathrm{P} /(\varepsilon \mathrm{L})$, where $\mathrm{P}$ is the product withdrawal rate, and $\mathrm{L}$ the total interstage flow rate at stage $s$. In a square cascade, $L$ is independent of $s$. Under the total reflux, $L$ is also equal to twice the reflux rate.

The solution of Eq. (156) by Cohen is in the form of Eq. (157) for the case of $\mathrm{N} \ll 1$ and the following intial and boundary conditions; $N(s, t=0)=N_{0}$ for all s-values, $N(0, t)=N_{0}$ at all $t, P=0$ at all $t$, and the holdup in the product refluxer $=H$ :

$$
\frac{N(n, l)-N_{0}}{N_{-}-N_{0}}=1-A e^{-B \tau},
$$

where $N_{-}=e^{\imath \|}$ which is the steady state atom fraction at the product end, $\tau=t / \lambda_{n}^{2}$ is a dimensionless time, and both $A$ and $B$ depend on two dimensionless parameters; $A=A\left(\varepsilon n, K / \lambda_{n}\right)$ and $B=B(\varepsilon n, K / \lambda n)$, in which $K=2 H / L$ and $K / \lambda_{n}=H / L h n$. Cohen mapped $A$ and $B$ for $K / \lambda_{n}=0.1(0.1) 0.5$ and for $\varepsilon n=$ $0.0(0.1) 1.2$ and stated that this solution is applicable to $t>\lambda n^{2} / 10$.

We extended the time limit to well below $\lambda \mathrm{n}^{2} / 10$ by deriving a higher-order solution of Eq. (156) in the form,

$$
\frac{N(n, t)-N_{0}}{N_{n}-N_{0}}=1-A_{1} e^{-B_{1} \tau}-A_{2} e^{-B_{2} \tau},
$$


and tabulated $A_{1}, B_{1}, A_{2}$ and $B_{2}$ for $K / \lambda_{n}=0.0(0.05) 0.5$ and $\varepsilon n=-1.2(0.2)$ 1.2. The relative importance of the second-order term is demonstrated by Figure $37 .^{(9)}$ The Figure shows that the second-order term is significant even at the time, $t$, which is within the Cohen's limit of $\tau>0.1$.

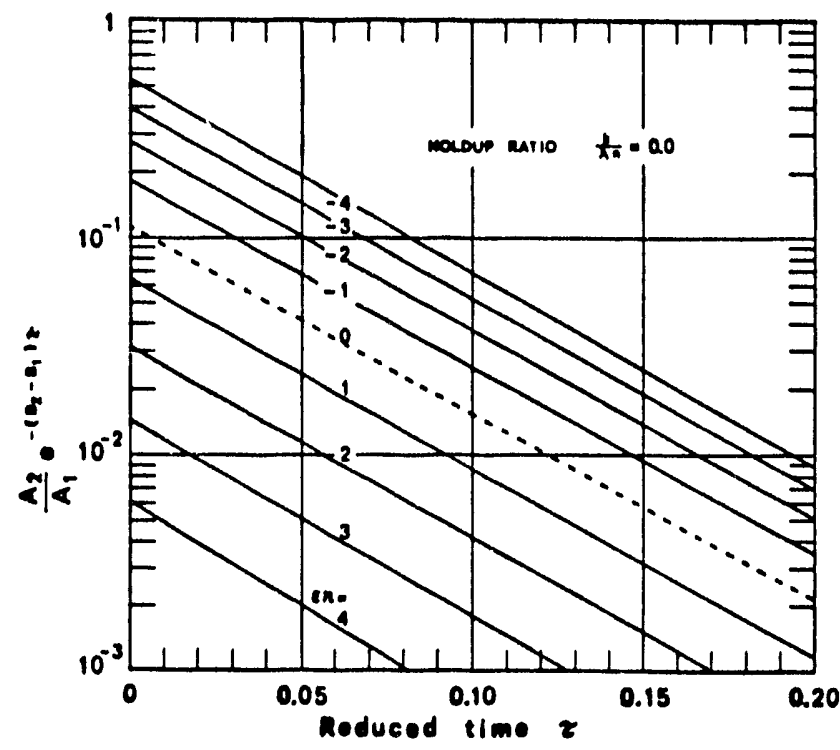

Figure 37. Relative importance of the fint and eccond terms of Eq. (158).

In particular, the second-order term is very significant in all cases of inverse isotope effect. The isotope of light elements that we are usually interested in enriching, e.g., $,{ }^{13} \mathrm{C},{ }^{15} \mathrm{~N},{ }^{34} \mathrm{~S}$, is heavier than the naturally most abundant isotopes. Thus, for instance, in a distillation process, the desired isotope usually enriches in the liquid phase, which corresponds to a positive en and to a normal vapor pressure isotope effect. If the VPIE is inverse, then $2 n$ is negative, and the second term of the solution, Eq. (37), is not at all negligible.

More generally, the transient behavior of a separation cascade is not symmetric with respect to change of signs in $\varepsilon$. The importance of this fact is illustrated by Figure $38^{(9)}$, in which the time it takes for the plant to attain $95 \%$ of a steady state separation, $\tau_{0.95}$, is plotted as a function of $\varepsilon_{n}$ and $k / \lambda n$. The transient time is a lot shorter in the domain of inverse isotope effect than in the domain of normal isotope effect.

Among other implications of this finding, a plant based on an inverse isotope effect will take a lot shorter time to reach a steady state and thus allow a product withdrawal sooner than a plant based on a normal isotope effect having the same absolute magnitude of separation factor and the same number of separative stages. ${ }^{(10)}$ Besides, it is easier to construct a product-end refluxer with low refluxer-holdup, if the desired isotope enriches in the gas phase. 


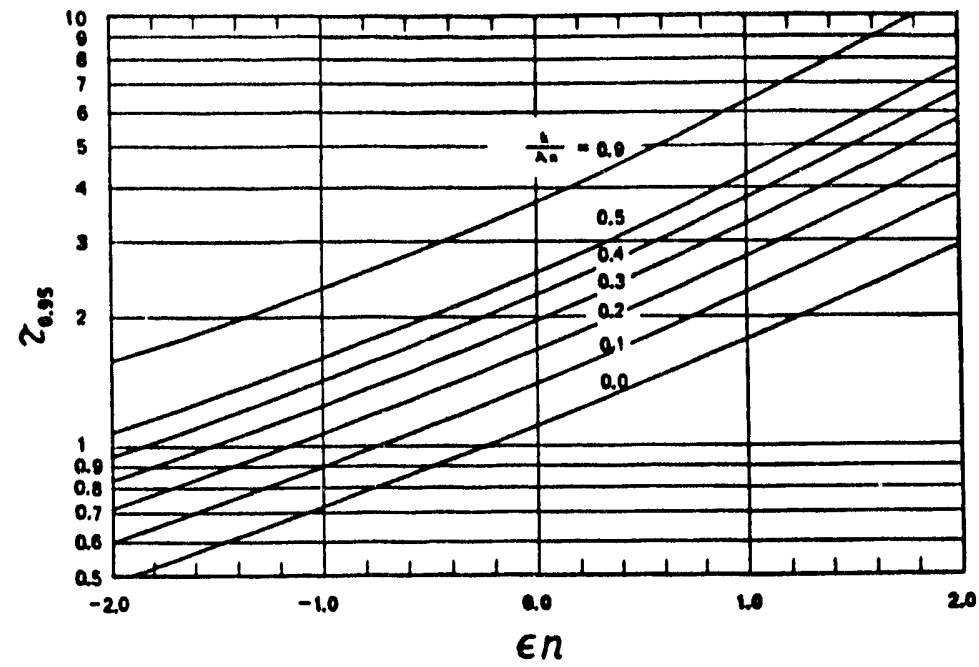

Figure 38. The $95 \%$ equilibrium time 
[ blank page ] 


\section{References}

101. Bigeleisen, J.; Mayer, M. G., J. Phys. Chem. 1947, 15, 261.

102. Bigeleisen, J., J. Phys. Chem. 1963, 38, 769.

103. Bigeleisen, J., "Proceedings of the Amsterdam Symposium on Isotope Separation," p. 121, 1991, North-Holland Publishing Company, Amsterdam.

104. Lanczos, C., "Applied Analysis," 1956, Prentice Hall, Englewood Cliffs, New Jersey.

105. Bigeleisen, J.; Ishida, T. J. Chem. Phys. 1968, 48, 1311.

106. Ishida, T.; Spindel, W.; Bigeleisen, J., Adv. Chem. Ser., 1969, 89, 192.

107. Bigeleisen, J.; Ishida, T.; Spindel, W., Proc. Natl. Acad. Sci. USA 1970, 67, 113.

108. Wilson, E. B.; Decius, J. C.; Cross, P. C., "Molecular Vibrations," 1955, McGraw-Hill, New York.

109. Bigeleisen, J., J. Chem. Phys. 1958, 28, 694.

110. Bigeleisen, J., J. Chem. Phys. 1955, 23, 2264.

111. Singh, G.; Wolfsberg, M., J. Chem. Phys. 1976, 62, 4165: Skaron, S. A.; Wolfsberg, M., J. Am. Chem. Soc. 1977, 99, 5253.

112. Decius, J. C.; Wilson, E. B., Jr., J. Chem. Phys. 1951, 19, 1409.

113. Bernstein, H. J.; Pullin, A. D. E., J. Chem. Phys. 1953, 21, 2188.

114. Pitzer, K. S., J. Chem. Phys. 1937, 5, 473, ibid., 1940, 8, 711, Chem. Rev. 1940, $27,39$.

115. Cottrell, T. L. J. Chem. Soc. 1948, 1448.

116. Pitzer, K. S.; Catalano, E. J. Am. Chem. Soc. 1956, 78, 4844.

117. Bernstein, H. J. J. Chem. Phys. 1952, 20, 263, 1328.

118. Evans, J. C.; Bernstein, H. J. Can. J. Chem. 1955, 33, 1171.

119. Bernstein, H. J. Can. J. Chem. 1956, 34, 617.

120. Bernstein, H. J. J. Chem. Phys. 1956, 24, 911.

121. Bernstein, H. J. J. Phys. Chem. 1965, 69, 1550.

122. Fujimoto, T.; Shingu, H. J. Chem. Soc. Jpn, 1962, 83, 19.

123. Hartshorn, S. R.; Shiner, V. J., Jr., J. Am. Chem. Soc. 1972, $94,9002$.

124. Wolfsberg, M., J. Chim. Phys. 1963, 60, 15.

125. Bigeleisen, J.; Goldstein, P., Z. Naturforsch., 1963, A18, 205.

126. Bigeleisen, J.; Weston, R. E., Jr.;Wolfsberg, M., Z. Naturforsch. 1963, A18, 210.

127. Person, W. B.; Newton, J. H., J. Chem. Phys. 1974, 61, 1040. 
128. Pauling, L., "The Nature of the Chemical Bond," 3rd ed., 1960, Cornell University, NY.

129. Wilmshurst, J. K.; J. Chem. Phys. 1958, 28, 733.

130. Inomata, N.; Masuda, S., Chem. Lett., 1982, 1003.

131. Boyd, R. J.; Edgecombe, K. E., J. Am. Chem. Soc. 1988, 110, 4182.

132. Luo, Y. R.; Benson, S. W., J. Phys. Chem. 1989, 93, 3306.

133. Gordy, W., Phys. Rev. 1946, 61, 604.

134. Wilmshurst, J. K., J. Chem. Phys. 1957, 27, 1129.

135. Jancso, G.; Van Hook, W. A., Chem. Rev. 1974, 74, 691.

136. Armstrong, G. T.; Brickwedde, F. G.; Scott, R. B., J. Res. Natl. Bur. Stand. 1955, 55, 39.

137. Johns, T. F., "Proceedings of the International Symposium on Isotope Separation," 1958, North-Holland Publishing Co., Amsterdam.

138. Bigeleissen, J.; Cragg, C. B.; Jeevanandam, M., J. Chem. Phys. 1967, 47, 4335.

139. Kirshenbaum, I.;Urey, H. C., J. Chem. Phys. 1942, 10, 706.

140. Wolff, H.; Höpfner, A., Ber. Bunsenges. Phys. Chem. 1969, 73, 480.

141. Thode, H. G., J. Am. Chem. Soc. 1940, 62, 581.

142. Armstrong, G. T., Natl. Bur. Standards Report No. 2626, June, 1953.

143. Overstreet, R.; Giauque, W. F., J. Am. Chem. Soc. 1937, 59, 254.

144. Spirko, V., J. Mol. Spectrosc. 1983, 101, 30.

145. Stern, M. J.; Van Hook, W. A.; Wolfsberg, M., J. Chem. Phys. 1963, 39, 3179.

146. Bader, R. F. W.; Beddall, P. M., J. Chem. Phys. 1972, 56, 3320.

147. Bader, R. F. W.; Beddall, P. M.; Peslak, J., Jr., J. Chem. Phys. 1973, 58, 557: Bader, R. F. W.; Henneker, W. H.; Cade, P. E., ibid. 1967, 46, 3341.

148. Ishida, T.; Spindel, W., J. Chem. Eng. Data, 1970, 15 (No. 1), 107.

149. Eshelman, D. M.; Torre, F. J.; Bigeleisen, J., J. Chem. Phys. 1974, 60, 420.

150. Monse, E. U.; Kaunder, L. N.; Spindel, W., J. Chem. Phys. 1964, 41, 3898: Monse, E. U.; Kauder, L. N.; Spindel, W., Z Naturforsch. 1963, 18a, 235: Monse, E. U.; Spindel, W.; Kauder, L. N.; Taylor, T. I. J Chem. Phys. 1960,32, 1557.

151. Willman, K. W.; Murray, R. W., Anal. Chem. 1983, 55, 1139.

152. HemandezR; Diaz, A. F.; Waltman, R.; Bargon, J. J. Phys. Chem. 1984, 88, 3333.

153. Dao, W. H.; Kuwana, T., J. Am. Chem. Soc. 1984, 106, 473.

154. Weisshaar, D. E.; Kuwana, T., J. Electroanal. Chem. 1984, 163, 395.

155. Bookbinder, D. C.; Bruce, J. A.; Dominey, R. N.; Lewis, N. S.; Wrighton, M. S. Proc. Natl. Acad. Sci. USA 1980, 77, 6280.

156. Tourillon, G.; Garnier, F., J. Phys. Chem. 1984, 88, 5281.

157. Hammett, L. P., J. Am. Chem. Soc. 1924, 46, 7. 
158. Kinoshita, K. in Modern Aspects of Electrochemistry; 1982, Bockris, J. O'M., Ed., Vol. 14, Chapter 8, Plenum, New York.

159. Chialvo, A. C.; Triaca, W. E.; Arvia, A. J. J. Electroanal. Chem. 1983, 146, 93.

160. Bilmes, S. A.; De Taconi, N. R.; Arvia, A. J. J. Electroanal. Chem. 1984, 164, 129.

161. Conway, B. E.; Angerstein-Kozlowska, H.; Sharp, W. B. A.; Cridulle, E. E., Anal. Chem. 1973, 45, 1331.

162. Krebs, L. C.; Ishida, T.; "Characterization of Electrochemically Modified Polycrostalline Platinum Surfaces," 1991, U.S. Department of Energy Report, DOE/ER/13855-6.

163. Delahay, P.; Mattax, C. C., J. Am. Chem. Soc. 1954, $26,874$.

164. Schiavon, G.; Zecchin, S.; Zotti, G.; Cattarin, S., J. Electroanal. Chem. 1986, 213, 53.

165. Brown, A. P.; Koval, C.; Anson, F. C., J. Electroanal. Chem. 1976, 72, 379.

166. Rousseau, K.; Farrington, G. C.; Dolphin, D., J. Org. Chem. 1972, 37 (24), 3968.

167. Aeiyach, S.; Dubois, J. E., Lacaze, P. C., J. Chem. Soc., Chem. Commun. 1986, 1608: Aeiyach, S.; Lacaze, P. C., J. Poly. Sci, Part A: Poly. Chem. 1989, $27,515$.

168. Jayanta, P. S.; Ishida, T., "Preparation of Polymer-Modified Electrodes: A Literature and Experimental Study," 1991, U. S. Department of Energy Report, DOE/ER/13855-5.

169. Minick, S. K.; Ishida, T., "Capacitance of Edge Plane of Pyrolytic Graphite in Acetonitrile Solutions," 1991, U. S. Department of Energy Report, DOE/ER/13855-4.

170. Bigeleisen, J.; Brooks, F. P.; Ishida, T.; Ribnikar, S. V., Rev. Sci. Instr. 1968, 39, 356.

171. Gagnon, E. G., J. Electrochem. Soc. 1974, 121 (11), 1444: Gagnon, E. G., ibid., 1975, 122 (4), 525: Gagnon, E. G. ibid., 1974, 121, 512: Austin, L. G.; Gagnon, E. G., ibid. 1973, $120(2), 251$.

172. Tobias, H.; Soffer, A., J. Electroanal. Chem. 1983, 148, 221: Oren, Y.; Soffer, A., ibid. 1985, 186, 63.

173. Cohen, K., "The Theory of Isotope Separation as Applied to the Large-Scale Production of U-235," 1951, McGraw-Hill, New York. 

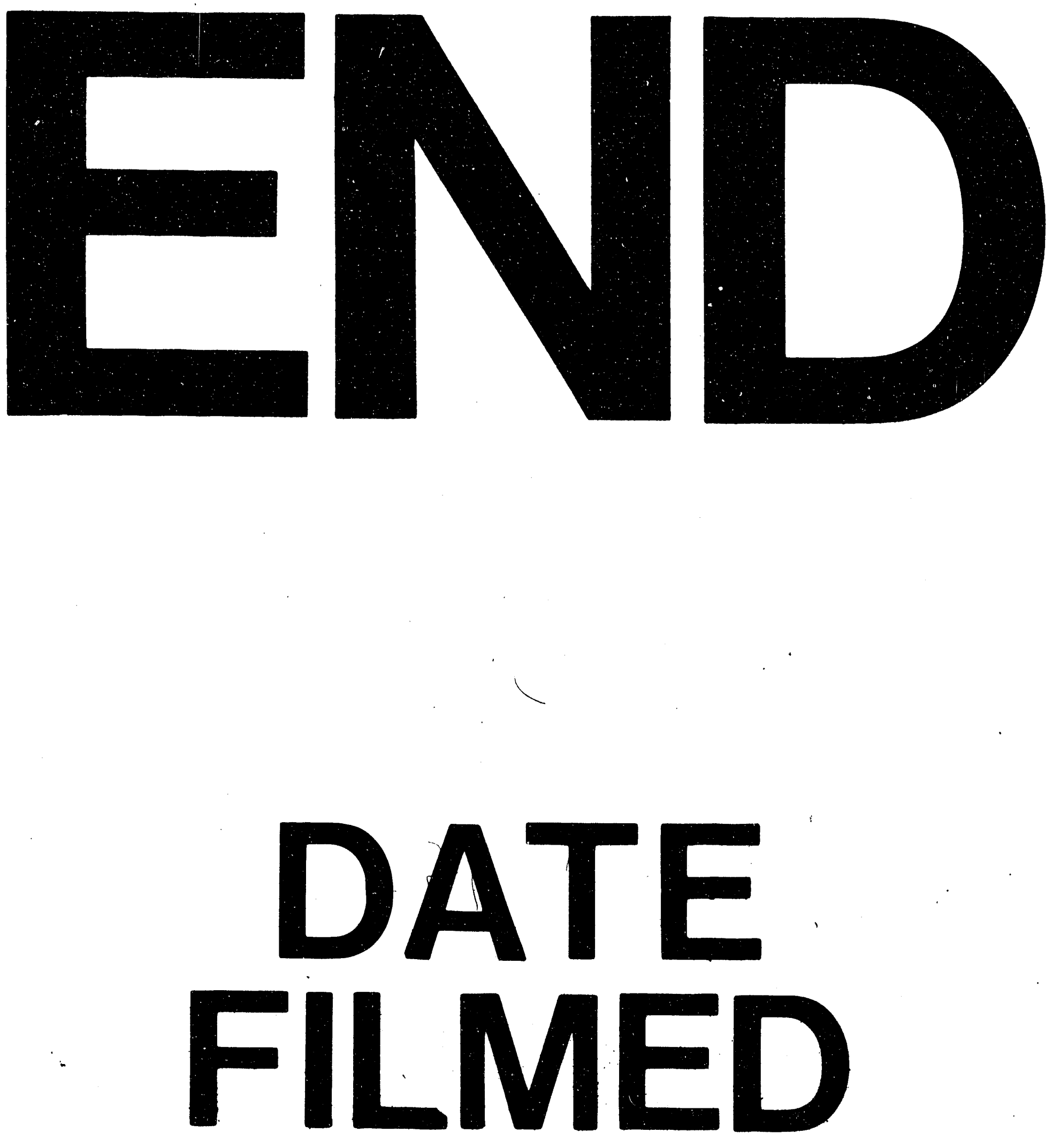

$\beta$

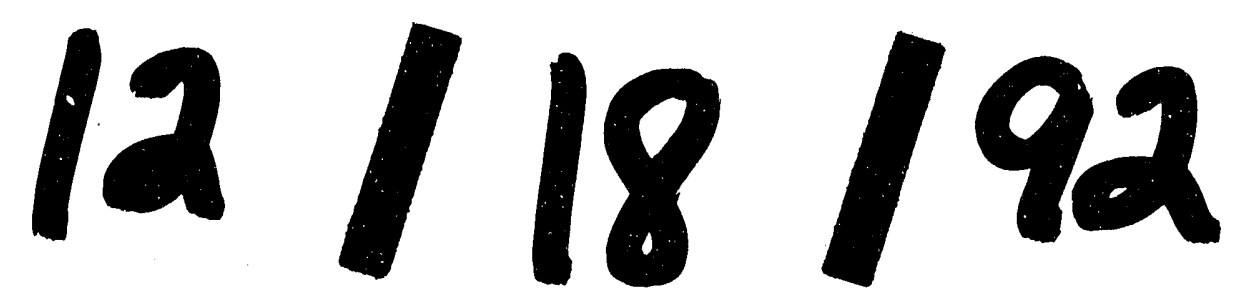


